TRANSACTIONS OF THE

AMERICAN MATHEMATICAL SOCIETY

Volume 342, Number 1, March 1994

\title{
WEIGHTS FOR CLASSICAL GROUPS
}

\author{
JIANBEI AN
}

\begin{abstract}
This paper proves the Alperin's weight conjecture for the finite unitary groups when the characteristic $r$ of modular representation is odd. Moreover, this paper proves the conjecture for finite odd dimensional special orthogonal groups and gives a combinatorial way to count the number of weights, block by block, for finite symplectic and even dimensional special orthogonal groups when $r$ and the defining characteristic of the groups are odd.
\end{abstract}

\section{INTRODUCTION}

Let $G$ be a finite group and $r$ a prime. A weight of $G$ is a pair $(R, \varphi)$ of an $r$-subgroup $R$ of $G$ and an irreducible character $\varphi$ of $N(R)$ such that $\varphi$ is trivial on $R$ and in an $r$-block of defect 0 of $N(R) / R$, where $N(R)=N_{G}(R)$ is the normalizer of $R$ in $G$. A radical subgroup $R$ of $G$ is an $r$-subgroup of $G$ such that $R=O_{r}(N(R))$, where $O_{r}(N(R))$ is the largest normal $r$-subgroup of $N(R)$. If $(R, \varphi)$ is a weight of $G$, then $R$ is necessarily a radical subgroup of $G$. A weight $(R, \varphi)$ is a $B$-weight for an $r$-block $B$ of $G$ if $\varphi$ is contained in an $r$-block $b$ of $N(R)$ such that $B=b^{G}$, that is, $B$ corresponds to $b$ by the Brauer homomorphism. In his paper [2], Alperin introduced the concept of weight in the modular representation theory of finite groups and conjectured that the number of weights of $G$ should equal the number of modular irreducible representations. Moreover, this equality should hold block by block. Here a weight $(R, \varphi)$ is identified with its conjugates in $G$. Alperin and Fong in [3] have proved this conjecture for symmetric groups and for finite general linear groups when the characteristic $r$ of modular representation is odd. The author in $[4,5]$ proved the conjecture for finite general linear and unitary groups when $r$ is even. In this paper, we prove the conjecture for the finite unitary groups when $r$ is odd. Moreover, we prove the conjecture for odd dimensional special orthogonal groups and give a combinatorial way to count the number of weights, block by block, for both finite symplectic and even dimensional special orthogonal groups when $r$ and the defining characteristic $p$ of groups are odd. We may suppose $p$ is different from $r$ since the result is known when $p$ is $r$ (see [2]).

In the first two sections, we describe the local structures of radical subgroups of a finite classical group, and in $\S 3$ we count the number of weights when the center of a radical subgroup is cyclic. The conjecture has been proved for unitary groups in (4D) and for odd dimensional special orthogonal groups in

Received by the editors August 26, 1991 and, in revised form, December 2, 1991.

1991 Mathematics Subject Classification. Primary 20C20; Secondary 20G40. 
(4G) and its remarks. Finally, the numbers of weights for symplectic and even dimensional special orthogonal groups have been counted in $(4 \mathrm{~F})$ and $(4 \mathrm{H})$ respectively.

I wish to express my sincere thanks to Professor Paul Fong, my Ph.D. advisor, for many corrections and suggestions. I also wish to thank Professor Bhama Srinivasan for a lot of help.

\section{THE GROUPS OF SYMPLECTIC TYPE}

Throughout this paper we shall follow the notation of [3, 5, 7], and [12]. In particular, $r$ is an odd prime and $E$ is an extraspecial $r$-group of order $r^{2 \gamma+1}$ with center $Z(E)=\langle y\rangle$. Then $E=\left\langle x_{1}, x_{2}, \ldots, x_{2 \gamma-1}, x_{2 \gamma}\right\rangle$ such that $\left[x_{2 i-1}, x_{2 i}\right]=x_{2 i-1}^{-1} x_{2 i}^{-1} x_{2 i-1} x_{2 i}=y,\left[x_{2 i}, x_{2 i+1}\right]=1$, for $1 \leq i \leq \gamma$, $\left[x_{i}, x_{j}\right]=1$ for $|i-j| \geq 2, x_{i}^{r}=1$ for $i \neq 2$. Thus $E$ has exponent $r$ or $r^{2}$ according as $x_{2}^{r}=1$ or $y$. An $r$-group $R$ is of symplectic type if $R$ is a central product of a nontrivial cyclic $r$-group $Z$ and an extraspecial group $E$, where $Z(E)$ is identified with $\Omega_{1}(Z)$. If $R>E$, then $R$ can be rewritten as the central product of $Z$ and an extraspecial group of exponent $r$, so that we may suppose $E$ has exponent $r$ and $E=\Omega_{1}(R)$. Thus we may always suppose $E$ is characteristic in $R$. Let Aut $R$ be the automorphism group of $R$, Inn $R$ the group of inner automorphisms, and Aut ${ }^{0} R=\{\sigma \in$ Aut $R:[\sigma, Z]=$ 1). Since every $\sigma$ in Aut ${ }^{0} R$ restricts to an element Aut ${ }^{0} E$ and every $\sigma$ in Aut $^{0} E$ extends to an element of Aut $^{0} R$, it follows that $\operatorname{Aut}^{0} R=$ Aut $^{0} E$. Denote

$$
K= \begin{cases}\operatorname{Sp}(2 \gamma, r) & \text { if } E \text { has exponent } r, \\ \operatorname{Sp}(2 \gamma-2, r) \ltimes r^{2(\gamma-1)+1} & \text { if } E \text { has exponent } r^{2},\end{cases}
$$

where $r^{2(\gamma-1)+1}$ denotes the extraspecial group of order $2(\gamma-1)+1$ and exponent $r$, and $\operatorname{Sp}(0, r) \ltimes r^{1}$ is interpreted as a group of order $r$. By [20, Theorem 1 or 15, p. 404] Aut $^{0} E=K \ltimes \operatorname{Inn} E$ (see also [3, p. 10]). In the following we shall consider the embeddings of $R$ into classical groups and determine the local structures of these embeddings.

Let $\mathbb{F}_{q}$ be the field of $q$ elements and $\eta= \pm 1$ a sign, where $q$ is a power of prime $p$ distinct from $r$. We first consider the embedding of $E$ in the groups $G=\mathrm{GL}(n, \eta q)$. Here following [7], we denote $\mathrm{U}(n, q)$ by $\mathrm{GL}(n,-q)$. The proofs of the following two lemmas are similar to that of [5, (1D), (1E), and $(1 \mathrm{~F})]$ and in the proofs such terms as orthogonal, orthonormal, and isometric will have meaning only in contexts involving $\mathrm{U}(n, q)$ and unitary spaces, but no meaning in contexts involving $\operatorname{GL}(n, q)$ and linear spaces.

(1A). Let $E$ be an extraspecial group of order $r^{2 \gamma+1}$ and $G=\mathrm{GL}\left(r^{\gamma}, \eta q\right)$. If $r$ divides $q-\eta$ (written $r \mid q-\eta$ ), then $G$ contains a unique conjugacy class of subgroups isomorphic to $E$. Moreover, if $r \mid q-1$, then $\mathbb{F}_{q}$ is a splitting field of E.

Proof. Given $1 \leq i \leq \gamma$, let $E_{i}=\left\langle x_{2 i-1}, x_{2 i}\right\rangle$, and $V_{i}$ a linear space of dimension $r$ over $\mathbb{F}_{q}$ or a unitary space of dimension $r$ over $\mathbb{F}_{q^{2}}$ according as $\eta=1$ or -1 . Then $E_{i}$ acts faithfully, irreducibly, and isometrically on $V_{i}$. Namely, let $w$ be an $r$ th root of unity in $\mathbb{F}_{q^{2}}$ and $\left\{v_{1}^{i}, v_{2}^{i}, \ldots, v_{r}^{i}\right\}$ an orthonormal basis of $V_{i}$. If $E$ has exponent $r$, then define

$$
x_{2 i-1}: v_{j}^{i} \mapsto w^{j} v_{j}^{i}, \quad x_{2 i}: v_{j}^{i} \mapsto v_{j+1}^{i},
$$


where $1 \leq j \leq r$. If $E$ has exponent $r^{2}$, then define

$$
x_{2 i-1}: v_{j}^{i} \mapsto w^{j} v_{j}^{i}, \quad x_{2 i}: v_{j}^{i} \mapsto \begin{cases}w v_{1}^{1} & \text { if } i=1 \text { and } j=r, \\ v_{j+1}^{i} & \text { otherwise, }\end{cases}
$$

where $1 \leq j \leq r$. Here subscripts on basis vectors are naturally read modulo $r$. In particular, $y: v_{j}^{i} \mapsto w v_{j}^{i}$ for all $j$.

Since $E$ is the central product of the $E_{i}$ 's and the element $y$ in $Z\left(E_{i}\right)$ is represented on $V_{i}$ by the scalar matrix $w I, E$ acts faithfully and irreducibly on $V=V_{1} \otimes V_{2} \otimes \cdots \otimes V_{\gamma}$. To see that the actions are by isometries, we first simplify notation and write

$$
v_{j_{1}}^{1} \otimes v_{j_{2}}^{2} \otimes \cdots \otimes v_{j_{\gamma}}^{\gamma}=\left[j_{1}, j_{2}, \ldots, j_{\gamma}\right], \quad 1 \leq j_{i} \leq r .
$$

The $r^{\gamma}$ elements $\left[j_{1}, j_{2}, \ldots, j_{\gamma}\right]$ form an orthonormal basis for $V$. So

$$
\begin{aligned}
x_{2 i-1}:\left[j_{1}, j_{2}, \ldots, j_{\gamma}\right] & \mapsto w^{j_{i}}\left[j_{1}, j_{2}, \ldots, j_{\gamma}\right], \\
x_{2 i}:\left[j_{1}, j_{2}, \ldots, j_{\gamma}\right] & \mapsto\left[j_{1}, \ldots, j_{i-1}, j_{i}+1, j_{i+1}, \ldots, j_{\gamma}\right],
\end{aligned}
$$

except when $E$ has exponent $r^{2}$, in which case the actions of $x_{i}$ for $i \neq 2$ are given by (1.4) and

$$
x_{2}:\left[j_{1}, j_{2}, \ldots, j_{\gamma}\right] \mapsto \begin{cases}{\left[j_{1}+1, j_{2}, \ldots, j_{\gamma}\right]} & \text { if } j_{1} \neq r, \\ w\left[1, j_{2}, \ldots, j_{\gamma}\right] & \text { if } j_{1}=r .\end{cases}
$$

Since basic vectors are mapped onto orthonormal vectors by generating elements of $E, E$ acts on $V$ by isometries, so that $G$ contains a copy of $E$.

Suppose $r \mid q-1$. Replacing $w$ by $w^{k}$ for $1 \leq k<r$ in the proof above, we get $r-1$ faithful and irreducible representations of $E$. By [14, 5.5.4] $E$ has $r-1$ nonlinear characters and all linear characters are realizable over $\mathbb{F}_{q}$ since $E / Z(E)$ is an elementary abelian $r$-group. Thus $\mathbb{F}_{q}$ is a splitting field of $E$.

To prove the uniqueness, it suffices to show that if $E$ is embedded as a subgroup of $G$, then there exists an orthonormal basis of the underlying space $V$ such that (1.4) or (1.5) holds according as $E$ has exponent $r$ or $r^{2}$. By Schur's lemma $y=w^{k} I$ for some integer $1 \leq k<r$. We may suppose $y=w I$ since $E=\left\langle x_{1}, x_{2}^{k}, x_{3}, x_{4}^{k}, \ldots, x_{2 \gamma-1}, x_{2 \gamma}^{k}\right\rangle$ and $\left[x_{2 i-1}, x_{2 i}^{k}\right]=y^{k}$.

Let $W_{j}=\left\{v \in V: x_{1} v=w^{j} v\right\}$ for $1 \leq j \leq r$. Then $V$ is the orthogonal sum of the $W_{j}$, so the $W_{j}$ for $1 \leq j \leq r$ are nondegenerate subspaces of $V$ and they are permuted by $x_{2}$ cyclically

$$
x_{2} W_{1}=W_{2}, \quad x_{2}^{2} W_{1}=W_{3}, \ldots, x_{2}^{r} W_{1}=W_{1},
$$

since $x_{1} x_{2}=w x_{2} x_{1}$. In particular, $W_{j}$ for $1 \leq j \leq r$ have the same dimension.

If $\gamma=1$ and $\left\{v_{1}\right\}$ is an orthonormal basis of $W_{1}$, then $\left\{v_{1}, x_{2} v_{1}, \ldots\right.$, $\left.x_{2}^{r-1} v_{1}\right\}$ is an orthonormal basis of $V$ and the actions of $x_{1}$ and $x_{2}$ on the basis are given by (1.2) or (1.3) according as $E$ has exponent $r$ or $r^{2}$. If $\gamma \geq 2$, then $L=\left\langle x_{3}, x_{4}, \ldots, x_{2 \gamma}\right\rangle$ is an extraspecial group of order $r^{2 \gamma-1}$ and exponent $r$ acting faithfully on $W_{1}$. We may suppose by induction that $x_{3}, x_{4}, \ldots, x_{2 \gamma}$ act on $W_{1}$ by (1.4) relative to the orthonormal basis $\left\{\left[j_{2}, j_{3}, \ldots, j_{\gamma}\right]\right\}$ of $W_{1}$, where $1 \leq j_{i} \leq r$. Thus $\left\{\left[j_{1}, j_{2}, \ldots, j_{\gamma}\right]=x_{2}^{j_{1}-1}\left[j_{2}, \ldots, j_{\gamma}\right]: 1 \leq j_{i} \leq r\right\}$ is an orthonormal basis of $V$ and $x_{1}, x_{2}, \ldots, x_{2 \gamma}$ act on the basis by (1.4) or (1.5). Thus any two embeddings of $E$ in $G$ are conjugate. 
Remark. (1) Suppose $r \mid q-\eta$ and $E$ is embedded in $G=\operatorname{GL}(n, \eta q)$ as a subgroup such that $y$ is represented by a scalar multiple of the identity matrix. Then $n=m r^{\gamma}$ for some integer $m \geq 1$, and there exists an orthonormal basis $\left\{\left[j_{1}, j_{2}, \ldots, j_{\gamma}\right]_{k}\right\}$ of the underlying space $V$ of $G$, where $1 \leq j_{i} \leq r$ and $1 \leq k \leq m$ such that for each $k$ the actions of $x_{2 i-1}$ and $x_{2 i}$ are given by (1.4) or $(1.5)$ with $\left[j_{1}, j_{2}, \ldots, j_{\gamma}\right]$ replaced by $\left[j_{1}, j_{2}, \ldots, j_{\gamma}\right]_{k}$. In particular, by (1A) such embedding of $E$ in $G$ is uniquely determined up to conjugacy in $G$. The proof of the remark is similar to that of the uniqueness of $(1 \mathrm{~A})$ and Remark (2) of [5, (1D)].

(2) Suppose $r \mid q-\eta, E$ has exponent $r$, and $E$ is embedded in $\operatorname{GL}\left(r^{\gamma}, \eta q\right)$ as a subgroup. In the notation of $(1 \mathrm{~A})$, we claim that $V$ has an orthonormal basis $\left\{\left[j_{1}, j_{2}, \ldots, j_{\gamma}\right]^{\prime}\right\}$, where $1 \leq j_{i} \leq r$ such that the actions of $x_{2 i-1}$ and $x_{2 i}$ for $i \geq 2$ are given by $(1.4)$ with $\left[j_{1}, j_{2}, \ldots, j_{\gamma}\right]$ replaced by $\left[j_{1}, j_{2}, \ldots, j_{\gamma}\right]^{\prime}$, and

$$
\begin{aligned}
& x_{1}:\left[j_{1}, j_{2}, \ldots, j_{\gamma}\right]^{\prime} \mapsto\left[j_{1}+1, j_{2}, \ldots, j_{\gamma}\right]^{\prime}, \\
& x_{2}:\left[j_{1}, j_{2}, \ldots, j_{\gamma}\right]^{\prime} \mapsto w^{-j_{1}}\left[j_{1}, j_{2}, \ldots, j_{\gamma}\right]^{\prime} .
\end{aligned}
$$

Indeed let $V_{j}^{\prime}=\left\{v \in V: x_{2} v=w^{-j} v\right\}$ for $1 \leq j \leq r$. Then $V_{j}^{\prime}$ are nondegenerate subspaces permuted by $x_{1}$ cyclically. If $\gamma=1$ and $\left\{v_{1}\right\}$ is an orthonormal basis of $V_{1}^{\prime}$, then $\left\{\left[j_{1}\right]^{\prime}=x_{1}^{j_{1}-1} v_{1}\right\}$, where $1 \leq j_{1} \leq r$, is a required basis. Suppose $\gamma \geq 2$ and $\left\{\left[j_{2}, j_{3}, \ldots, j_{\gamma}\right]^{\prime}\right\}$, where $1 \leq j_{i} \leq r$, is an orthonormal basis of $V_{1}^{\prime}$ such that the actions of $x_{3}, \ldots, x_{2 \gamma}$ on the basis are given by $(1.4)$ with $\left[j_{2}, j_{3}, \ldots, j_{\gamma}\right]$ replaced by $\left[j_{2}, j_{3}, \ldots, j_{\gamma}\right]^{\prime}$. Let $\left[j_{1}, j_{2}, \ldots, j_{\gamma}\right]^{\prime}=x_{1}^{j_{1}-1}\left[j_{2}, \ldots, j_{\gamma}\right]^{\prime}$. Then $\left\{\left[j_{1}, j_{2}, \ldots, j_{\gamma}\right]^{\prime}: 1 \leq j_{i} \leq r\right\}$ is a required basis.

(1B). Suppose $r \mid q-\eta$. Let $G=\mathrm{GL}\left(r^{\gamma}, \eta q\right)$ and $R=Z E$ an $r$-subgroup of symplectic type of $G$, where $Z=Z(G)_{r}$ and $E$ is an extraspecial subgroup of order $r^{2 \gamma+1}$ of $G$. Set $C=C_{G}(R)$ and $N=N_{G}(R)$. Then $C=Z(G)=Z(N)$ and if $E$ has exponent $r$, then $N / R C \simeq \operatorname{Sp}(2 \gamma, q)$. In addition, if $R$ is radical in $G$, then $E$ has exponent $r$. Moreover, each linear character of $Z(N)$ acting trivially on $O_{r}(Z(N))$ has an extension to $N$ trivial on $R$.

Proof. By (1A) $\mathbb{F}_{q^{2}}$ is a splitting field, so that $C=Z(G)=Z(N)$. The proof of the last assertion is the same as that of [5, (1E)] with 2 replaced by $r$. If $R>E$, then $E$ may be assumed to have exponent $r$. The elements of $N$ induce automorphisms in $\mathrm{Aut}^{0} E=\mathrm{Aut}^{0} R$. Suppose $E$ has exponent $r$ and acts on the underlying space $V$ of $G$ by (1.4). We shall exhibit elements in $N$ which together with $R$ generate Aut $^{0} E$.

(1) Let $g$ be the element in $G$ such that

$$
g:\left[j_{1}, j_{2}, \ldots, j_{i}, \ldots, j_{\gamma}\right] \mapsto\left[j_{i}, j_{2}, \ldots, j_{1}, \ldots, j_{\gamma}\right] .
$$

Then $g^{-1} x_{1} g=x_{2 i-1}, g^{-1} x_{2 i-1} g=x_{1}, g^{-1} x_{2} g=x_{2 i}, g^{-1} x_{2 i} g=x_{2}$, and $g^{-1} x_{k} g=x_{k}$ for all other indices. Thus $N$ contains a subgroup inducing the symmetric group $\mathbf{S}(\gamma)$ on the set $\left\{E_{1}, E_{2}, \ldots, E_{\gamma}\right\}$.

(2) Let $\left\{\left[j_{1}, j_{2}, j_{3}, \ldots, j_{\gamma}\right]^{\prime}\right\}$ be the orthonormal basis of $V$ given by Remark (2), and $g$ the element in $G$ such that

$$
g:\left[j_{1}, j_{2}, \ldots, j_{\gamma}\right]^{\prime} \mapsto\left[j_{1}, j_{2}, \ldots, j_{\gamma}\right] .
$$

Then $g^{-1} x_{1} g=x_{2}^{-1}, g^{-1} x_{2} g=x_{1}$, and $g^{-1} x_{k} g=x_{k}$ for $k \geq 3$. By (1) for each $1 \leq i \leq \gamma$, there exists $h \in G$ such that $h^{-1} x_{2 i-1} h=x_{2 i}^{-1}$, 
$h^{-1} x_{2 i} h=x_{2 i-1}$, and $h^{-1} x_{k} g=x_{k}$ for all other indices. Thus $N$ contains a subgroup inducing Weyl group of type $C_{\gamma}$ on $R / Z(R)$.

(3) Let $g$ be the element in $G$ such that

$$
g:\left[j_{1}, j_{2}, j_{3}, \ldots, j_{\gamma}\right] \mapsto\left[\lambda j_{1}, j_{2}, j_{3}, \ldots, j_{\gamma}\right],
$$

where $\lambda$ is a nonzero element of $\mathbb{Z} / \mathbb{Z} r$. Then $g^{-1} x_{1} g=x_{1}^{\lambda}, g^{-1} x_{2} g=x_{2}^{\lambda^{-1}}$, and $g^{-1} x_{k} g=x_{k}$ for $k>2$. In addition, let $g$ be the element in $G$ such that

$$
g:\left[j_{1}, j_{2}, j_{3}, \ldots, j_{\gamma}\right] \mapsto\left[j_{1}+j_{2}, j_{2}, j_{3}, \ldots, j_{\gamma}\right]
$$

Then $g^{-1} x_{1} g=x_{1} x_{3}, g^{-1} x_{4} g=x_{4} x_{2}^{-1}$, and $g^{-1} x_{k} g=x_{k}$ for all other indices. Since $\left\langle x_{1}, x_{3}, \ldots, x_{2 \gamma-1}\right\rangle$ and $\left\langle x_{2}, x_{4}, \ldots, x_{2 \gamma}\right\rangle$ give a hyperbolic decomposition of $R / Z(R)$, the element $g$ of (1.6) induces

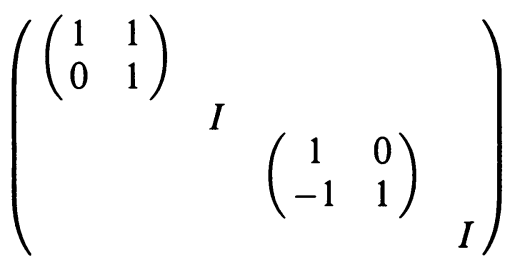

relative to this decomposition of $R / Z(R)$. By (1) we may replace $E_{1}$ and $E_{2}$ by $E_{i}$ and $E_{j}$ for $1 \leq i \neq j \leq \gamma$. Thus $N$ contains a subgroup inducing

$$
\left\langle\left(\begin{array}{cc}
A & \\
& \left(A^{-1}\right)^{t}
\end{array}\right): A \in \mathrm{GL}(\gamma, r)\right\rangle
$$

on $R / Z(R)$.

(4) We claim there are elements in $N$ inducing

$$
\left(\begin{array}{cc}
I & X \\
& I
\end{array}\right)
$$

on $R / Z(R)$ for any $X$ such that $X^{t}=X$. By (3) it suffices to show this when

$$
X=\operatorname{diag}\{1,0,0, \ldots, 0\} .
$$

Indeed, let $g$ be the element in $G$ such that

$$
g:\left[j_{1}, j_{2}, \ldots, j_{\gamma}\right] \mapsto w^{-\left(j_{1}+1\right) j_{1} / 2}\left[j_{1}, j_{2}, \ldots, j_{\gamma}\right],
$$

where $w$ is the $r$ th root of unity in $\mathbb{F}_{q^{2}}$ given by (1.4). Then $g^{-1} x_{2} g=x_{1} x_{2}$, and $g^{-1} x_{k} g=x_{k}$ for all other indices. Thus the claim holds.

By (3) and (4) $N$ contains a subgroup inducing a Borel subgroup of $\operatorname{Sp}(2 \gamma, r)$ on $R / Z(R)$. Thus $N$ induces $\operatorname{Sp}(2 \gamma, r)$ on $R / Z(R)$. Suppose $R$ is radical in $G$. If $E$ has exponent $r^{2}$, then $R=E$ and the element $g$ defined by (1.7) lies in $N \backslash R$. Moreover, as shown in the proof of [20, p. 166], $g$ induces an element of $Z(K)$, where $K \simeq \operatorname{Aut}^{0} E / \operatorname{Inn} E$ is given by (1.1). Let $Q=\langle g, E\rangle$, so that $Q \leq N$. We claim that $Q \leq O_{r}(N)$. Indeed for any $h \in N, h$ induces an element of Aut $^{0} E$. Replacing $h$ by $h x$ for some $x \in E$, we may suppose $h$ induces an element of $K$. Thus $[h, g]$ induces a trivial action on $E$ and then $[h, g] \in C=Z(G)$, so that $h g h^{-1}=z g$ for some $z \in C$ and $z \in O_{r}(C)=Z(R)$ since $z g$ and $g$ are $r$-elements. So $h$ normalizes $Q$ and 
the claim holds. It follows that $R$ is nonradical in $G$ and we may suppose $E$ has exponent $r$. This proves (1B).

We now consider the embedding of $R$ into finite classical groups. Let $G=$ $\mathrm{U}(n, q), \operatorname{Sp}(2 n, q), O(2 n+1, q)$, or $\mathrm{O}^{\eta}(2 n, q)$, and let $V$ be the underlying space of $G$, where $\eta= \pm 1$. If $V$ is a symplectic or orthogonal space, we always suppose the characteristic $p$ of $\mathbb{F}_{q}$ is odd. Moreover, we denote by $I(V)$ the group of isometries of $V, I_{0}(V)$ the subgroups of $I(V)$ of determinant 1 , and $\eta(V)$ the type of $V$ if $V$ is orthogonal. For simplicity, we set $\eta(V)=1$ if $V$ is symplectic.

We define the integers $e, a$, and $\operatorname{sign} \varepsilon= \pm 1$ as follows: In the case $G=$ $\mathrm{U}(n, q)$, let $e$ be the order of $-q$ modulo $r$ and $\varepsilon=1$ or -1 according as $e$ is even or odd; in the remaining cases, let $e$ be the order of $q^{2}$ modulo $r$ and $\varepsilon$ the sign chosen so that $r^{a}$ divides $q^{e}-\varepsilon$. In all cases, let $r^{a}$ be the exact power of $r$ dividing $q^{2 e}-1$. In the case $G=\mathrm{U}(n, q)$, our definition of $e$ above is different from that of $\left[11\right.$, p. 125]. In fact, if $r \mid q^{e}+1$, then our $e$ is the same as that of [11]. If $r \mid q^{e}-1$, then our $e$ is the double of that of [11].

We recall that there exists a set $\mathscr{F}$ of polynomials serving as elementary divisors for all semisimple elements of each of these groups. First suppose $G=$ $\mathrm{U}(n, q)$. For each monic polynomial $\Delta(X)=X^{m}+a_{m-1} X^{m-1}+\cdots+a_{1} X+a_{0}$ of $\mathbb{F}_{q^{2}}[X]$ with nonzero roots, let $\widetilde{\Delta}(X)=\left(a_{0}^{-1}\right)^{q} X^{m} \Delta^{q}\left(X^{-1}\right)$. Then define

$$
\begin{aligned}
& \mathscr{F}_{1}=\{\Delta: \Delta \text { is monic, irreducible, } \Delta \neq X, \Delta=\widetilde{\Delta}\}, \\
& \mathscr{F}_{2}=\{\Delta \widetilde{\Delta}: \Delta \text { is monic, irreducible, } \Delta \neq X, \Delta \neq \widetilde{\Delta}\},
\end{aligned}
$$

and $\mathscr{F}=\mathscr{F}_{1} \cup \mathscr{F}_{2}$. Suppose $G$ is a symplectic or orthogonal group. For each monic polynomial $\Delta(X)$ in $\mathbb{F}_{q}[X]$ with nonzero roots, let $\Delta(X)^{*}$ be the monic polynomial in $\mathbb{F}_{q}[X]$ whose roots are the inverses of the roots of $\Delta(X)$. Define

$\mathscr{F}_{0}=\{X-1, X+1\}$,

$\mathscr{F}_{1}=\left\{\Delta: \Delta\right.$ is monic, irreducible, $\Delta \neq X, \Delta \neq X \pm 1$, and $\left.\Delta=\Delta^{*}\right\}$,

$\mathscr{F}_{2}=\left\{\Delta \Delta^{*}: \Delta\right.$ is monic, irreducible, $\Delta \neq X, \Delta \neq X \pm 1$, and $\left.\Delta \neq \Delta^{*}\right\}$,

and $\mathscr{F}=\mathscr{F}_{0} \cup \mathscr{F}_{1} \cup \mathscr{F}_{2}$. Given $\Gamma \in \mathscr{F}$, denote $d_{\Gamma}$ its degree and $\delta_{\Gamma}$ its reduced degree defined by

$$
\delta_{\Gamma}= \begin{cases}d_{\Gamma} & \text { if } G=\mathrm{U}(n, q) \text { and } \Gamma \in \mathscr{F}_{1} \cup \mathscr{F}_{2}, \\ d_{\Gamma} & \text { if } G \neq \mathrm{U}(n, q) \text { and } \Gamma \in \mathscr{F}_{0}, \\ \frac{1}{2} d_{\Gamma} & \text { if } G \neq \mathrm{U}(n, q) \text { and } \Gamma \in \mathscr{F}_{1} \cup \mathscr{F}_{2} .\end{cases}
$$

Thus $\delta_{\Gamma}$ is an integer. We define a sign $\varepsilon_{\Gamma}$ for $\Gamma \in \mathscr{F}$ by

$$
\varepsilon_{\Gamma}= \begin{cases}\varepsilon & \text { if } \Gamma \in \mathscr{F}_{0} \\ -1 & \text { if } \Gamma \in \mathscr{F}_{1}, \\ 1 & \text { if } \Gamma \in \mathscr{F}_{2} .\end{cases}
$$

Given a semisimple element $s \in G$, there exists a unique orthogonal decomposition

$$
V=\sum_{\Gamma} V_{\Gamma}(s), \quad s=\prod_{\Gamma} s(\Gamma),
$$


where the $V_{\Gamma}(s)$ are nondegenerate subspaces of $V, s(\Gamma) \in \mathrm{U}\left(V_{\Gamma}(s)\right)$ or $I\left(V_{\Gamma}(s)\right)$ according as $V$ is or is not a unitary space, and $s(\Gamma)$ has minimal polynomial $\Gamma \in \mathscr{F}$. The decomposition (1.8) will be called the primary decomposition of $s$ in $G$. Let $m_{\Gamma}(s)$ be the multiplicity of $\Gamma$ in $s(\Gamma)$. Then

$$
C_{G}(s)=\prod_{\Gamma} C_{\Gamma}(s)
$$

where $C_{\Gamma}(s)=C_{\mathrm{U}\left(V_{\Gamma}(s)\right)}(s(\Gamma))$ or $C_{I\left(V_{\Gamma}(s)\right)}(s(\Gamma))$. Moreover, by [11, (1A)] or $[12,(1.13)]$

$$
C_{\Gamma}(s)= \begin{cases}I\left(V_{\Gamma}(s)\right) & \text { if } \Gamma \in \mathscr{F}_{0}, \\ \mathrm{GL}\left(m_{\Gamma}(s), \varepsilon_{\Gamma} q^{\left.\delta_{\Gamma}\right)}\right. & \text { if } \Gamma \in \mathscr{F}_{1} \cup \mathscr{F}_{2} .\end{cases}
$$

A semisimple element $s \in G$ is primary if $s=s(\Gamma)$.

Suppose $V$ is a symplectic or orthogonal space and $s$ decomposes as (1.8). Let $\eta_{\Gamma}(s)$ be the type of $V_{\Gamma}(s)$, where $\eta_{\Gamma}(s)=1$ for all $\Gamma \in \mathscr{F}$ if $V$ is symplectic. So $s$ lies in $I_{0}(V)$ if and only if $m_{X+1}(s)$ is even. By [12, (1.12)], the multiplicity and type functions $\Gamma \mapsto m_{\Gamma}(s), \Gamma \mapsto \eta_{\Gamma}(s)$ satisfy the following relations

$$
\begin{aligned}
\operatorname{dim} V & =\sum_{\Gamma} d_{\Gamma} m_{\Gamma}(s) \\
\eta(V) & =(-1)^{(q-1) / 2 m_{X-1}(s) m_{X+1}(s)} \prod_{\Gamma} \eta_{\Gamma}(s), \\
\eta\left(V_{\Gamma}(s)\right) & =\varepsilon_{\Gamma}^{m_{\Gamma}(s)} \quad \text { for } \Gamma \in \mathscr{F}_{1} \cup \mathscr{F}_{2}, \text { and } V \text { orthogonal. }
\end{aligned}
$$

Conversely, if $\Gamma \mapsto \eta_{\Gamma}, \Gamma \mapsto \eta_{\Gamma}$ are functions from $\mathscr{F}$ to $\mathbb{N},\{ \pm 1\}$ respectively satisfying (1.11) with $m_{\Gamma}(s)$ and $\eta_{\Gamma}(s)$ replaced by $n_{\Gamma}$ and $\eta_{\Gamma}$, then there exists a semisimple element $s$ of $I(V)$ with those functions as multiplicity and type functions. Moreover, two semisimple elements $s$ and $s^{\prime}$ of $I(V)$ are conjugate in $I(V)$ if and only if $m_{\Gamma}(s)=m_{\Gamma}\left(s^{\prime}\right)$ and $\eta_{\Gamma}(s)=\eta_{\Gamma}\left(s^{\prime}\right)$.

Let $Z=\langle z\rangle$ be a cyclic $r$-group of order $r^{a+\alpha}$ with $\alpha \geq 0, E$ an extraspecial $r$-group of order $r^{2 \gamma+1}$, and $R=Z E$ a group of symplectic type with $Z(R)=$ $Z$. Moreover, we may suppose $E$ has exponent $r$ if $R>E$.

(1C). Let $G=\mathrm{U}(n, q), \mathrm{Sp}(2 n, q), O(2 n+1, q)$, or $\mathrm{O}^{\eta}(2 n, q)$, where $\eta=$ \pm 1 . Suppose $\mathbf{F}$ and $\mathbf{F}^{\prime}$ are two embeddings of $R$ in $G$ such that $\mathbf{F}(z)$ and $\mathbf{F}^{\prime}(z)$ are primary elements of $G$. Then $n=$ mer $^{\alpha+\gamma}$ for some $m \geq 1, \mathbf{F}(R)$ and $\mathrm{F}^{\prime}(R)$ are conjugate in $G$, and $\eta=\varepsilon^{m}$ if $G=\mathrm{O}^{\eta}(2 n, q)$. Identify $R$ with $\mathbf{F}(R)$ and let $C=C_{G}(R), N=N_{G}(R)$, and $N^{0}=\{g \in N:[g, Z]=1\}$. Then $C \simeq \mathrm{GL}\left(m, \varepsilon q^{e r^{\alpha}}\right)$. Furthermore, suppose $R$ is a radical subgroup of $G$.

(1) $E$ has exponent $r$ and $N^{0}=L C$, where $R \unlhd L, L \cap C=Z(C)=$ $Z\left(C_{G}(z)\right)=Z(L), L / R Z(L) \simeq \operatorname{Sp}(2 \gamma, r)$, and $[C, L]=1$. Moreover, each linear character of $Z(L)$ acting trivially on $O_{r}(Z(L))$ can be extended as a character of $L$ acting trivially on $R$.

(2) $N / N^{0} \simeq N_{G}(Z) / C_{G}(Z)$ is cyclic of order er ${ }^{\alpha}$ or $2 e r^{\alpha}$ according as $G=\mathrm{U}(n, q)$ or $G \neq \mathrm{U}(n, q)$.

Proof. Since both $Z(\mathbf{F}(R))$ and $Z\left(\mathbf{F}^{\prime}(R)\right)$ are cyclic groups of order $r^{a+\alpha}$ generated by primary elements $\mathbf{F}(z)$ and $\mathbf{F}^{\prime}(z)$ respectively, they are conjugate in $G$, so that we may suppose $Z(\mathbf{F}(R))=Z\left(\mathbf{F}^{\prime}(R)\right)$. Thus $\mathbf{F}(E)$ and $\mathbf{F}^{\prime}(E)$ 
are subgroups of $C_{G}(\mathbf{F}(z))$. Let $H=C_{G}(\mathbf{F}(z))$ and $\Gamma$ be the unique elementary divisor of $\mathbf{F}(z)$. Then $H \simeq \mathrm{GL}\left(m_{\Gamma}(\mathbf{F}(z)), \varepsilon q^{e^{\alpha}}\right)$ and the two embeddings $\mathbf{F}(E)$ and $\mathrm{F}^{\prime}(E)$ of $E$ in $H$ can be viewed as embeddings of $E$ in $\mathrm{GL}\left(m_{\Gamma}(\mathbf{F}(z)), \varepsilon q^{e^{\alpha}}\right)$ in which a generator $y$ of $Z(E)$ is represented by scalar multiples of the identity matrix. It then follows by Remark (1) of (1A) that $\mathbf{F}(E)$ and $\mathbf{F}^{\prime}(E)$ are conjugate in $H$ and $m_{\Gamma}(\mathbf{F}(z))=m r^{\gamma}$ for some $m \geq 1$. So $\mathbf{F}(R)$ and $\mathbf{F}^{\prime}(R)$ are conjugate in $G$, and $\eta=\varepsilon^{m r^{\gamma}}=\varepsilon^{m}$ if $G=\mathrm{O}^{\eta}(2 n, q)$.

Identify $H$ with $\mathrm{GL}\left(m r^{\gamma}, \varepsilon q^{e r^{\alpha}}\right)$. Let $\mathbf{W}$ be the faithful and irreducible representation of $E$ in $\mathrm{GL}\left(r^{\gamma}, \varepsilon q^{e r^{\alpha}}\right)$ given by (1A), and let $L_{\gamma}$ be the normalizer of $\mathbf{W}(E)$ in $\mathrm{GL}\left(r^{\gamma}, \varepsilon q^{e r^{\alpha}}\right)$. Then the commuting algebras of $L_{\gamma}$ and $E$ on the underlying space of $\mathrm{GL}\left(r^{\gamma}, \varepsilon q^{e r^{\alpha}}\right)$ are $\mathbb{F}_{q^{e r^{\alpha}}}$ or $\mathbb{F}_{q^{2 e^{\alpha} \alpha}}$ according as $\varepsilon=1$ or -1 . Moreover, if $E$ has exponent $r$, then $L_{\gamma} / Z\left(L_{\gamma}\right) \simeq$ Aut $^{0} E$. By Remark (1) of $(1 \mathrm{~A}) \mathbf{F}(E)$ in $H$ can be viewed as an $m$-fold diagonal embedding of $E$ into $\mathrm{GL}\left(m r^{\gamma}, \varepsilon q^{e r^{\alpha}}\right)$ given by

$$
\left(\begin{array}{llll}
g & & & \\
& g & & \\
& & \ddots & \\
& & & g
\end{array}\right), \quad g \in \mathbf{W}(E) .
$$

In particular, $C=C_{H}(\mathbf{F}(R)) \simeq \mathrm{GL}\left(m, \varepsilon q^{e r^{\alpha}}\right)$. Let $L$ be the image of $L_{\gamma}$ under (1.12), so that $\mathbf{F}(R) \unlhd L, L \leq N^{0}=N_{H}(\mathbf{F}(R)), C_{H}(L)=C_{H}(E)=C$, and $[L, C]=1$. Suppose $\mathbf{F}(R)$ is radical in $G$ and $E$ has exponent $r^{2}$, so that $R=E$. As shown in the proof of (4) of (1B), there exists an $r$-element $x$ of $L_{\gamma}$ such that $x \notin \mathbf{W}(E)$ and $x$ induces an element of $Z\left(\operatorname{Aut}^{0} E / \operatorname{Inn} E\right)$, so that the image $w$ of $x$ under (1.12) is an $r$-element of $L \backslash \mathbf{F}(E)$. If $Q=\langle w, \mathbf{F}(E)\rangle$, then $C_{H}(\mathbf{F}(E))=C_{H}(Q)=C$. Since $N^{0} \unlhd N$ and $\mathbf{F}(E)$ is radical in $G$, it follows that $\mathbf{F}(E)=O_{r}\left(N^{0}\right)$ and each element of $N^{0}$ induces an element of Aut $^{0} E$, so that $w$ induces an element of $Z\left(\operatorname{Aut}^{0} E / \operatorname{Inn} E\right)$. We claim $Q \leq$ $O_{r}\left(N^{0}\right)$. Indeed for each $h \in N^{0}$, we may suppose $h$ induces an element of Aut ${ }^{0} E / \operatorname{Inn} E$ and then $[h, w]$ acts trivially on $E$, so that $[h, w] \in C$. Since $h$ normalizes $C$ and $w$ commutes with $C,[h, w]$ commutes with $C$ and $h w h^{-1}=g w$ for some $g \in Z(C)=Z(H)$. Since $g w$ and $w$ are commutative $r$-elements, $g$ is an $r$-element of $Z(H)$, so that $g \in O_{r}(H) \leq \mathbf{F}(E)$. Thus $h$ normalizes $Q$ and $Q \leq O_{r}\left(N^{0}\right)$. This is a contradiction and $E$ has exponent $r$.

Identify $R$ with $\mathbf{F}(R)$. Since $L / Z(L) \simeq \operatorname{Aut}^{0} R$ and $N^{0}$ induces a subgroup of Aut $^{0} R$, it follows that $N^{0}=L C$. Thus $Z(H) \leq Z\left(N^{0}\right) \leq Z(L) Z(C)$, $Z(L) \leq Z(C)=Z(H)$, and $L \cap C \leq Z(C)$, so that $Z(L)=Z(H)=Z(C)=$ $L \cap C$. The last assertion of (1) follows by (1B) since $L \simeq L_{\gamma}$. Finally, $N_{G}(Z) / C_{G}(Z)$ is cyclic of order $e r^{\alpha}$ or $2 e r^{\alpha}$ according as $G=\mathrm{U}(n, q)$ or $G \neq \mathrm{U}(n, q)$ by [11,(3D)] or [12, (5B)]. Suppose $g$ generates $N_{G}(Z)$ modulo $C_{G}(Z)$. Then $E$ and $g^{-1} E g$ are extraspecial subgroups of $H=C_{G}(Z)$, and they are conjugate in $H$ by Remark (1) of (1A), so that $h^{-1} g^{-1} E g h=E$ for some $h \in H$ and $g h \in N$. On the other hand, $N \leq N_{G}(Z)$ and $N^{0}=$ $N \cap C_{G}(Z)$, so that $N / N^{0} \simeq N_{G}(Z) / C_{G}(Z)$ and (1C) holds.

Remark. In the notation of (1C), let $E=\left\langle x_{1}, x_{2}, \ldots, x_{2 \gamma}\right\rangle, R^{\prime}=\left\langle x_{1}, x_{3}, \ldots\right.$, $\left.x_{2 \gamma-1}, Z\right\rangle$. Identify $R$ with $\mathbf{F}(R)$ and $R^{\prime}$ with $\mathbf{F}\left(R^{\prime}\right)$. Then $R^{\prime} \unlhd R$ and 
$C_{G}\left(R^{\prime}\right)=C_{1} \times C_{2} \times \cdots \times C_{r^{\gamma}}$ is a regular subgroup $G$, where $C_{i} \simeq \mathrm{GL}\left(m, \varepsilon q^{e r^{\alpha}}\right)$ for all $i$. Indeed by Remark (1) of (1A) we may suppose the underlying space of $H=C_{G}(Z)$ has an orthonormal basis $\left\{\left[j_{1}, j_{2}, \ldots, j_{\gamma}\right]_{k}\right\}$, where $1 \leq j_{i} \leq r$ and $1 \leq k \leq m$, such that the actions of $x_{1}, x_{2}, \ldots, x_{2 \gamma}$ on the basis are given by $(1.4)$ or $(1.5)$ with $\left[j_{1}, j_{2}, \ldots, j_{\gamma}\right]$ replaced by $\left[j_{1}, j_{2}, \ldots, j_{\gamma}\right]_{k}$. Thus each $x_{2 i-1}$ is a diagonal matrix with respect to the basis for $1 \leq i \leq \gamma$, so $C_{H}\left(R^{\prime}\right)=C_{G}\left(R^{\prime}\right)=C_{1} \times C_{2} \times \cdots \times C_{r^{\gamma}}$, where $C_{i} \simeq \mathrm{GL}\left(m, \varepsilon q^{e r^{\alpha}}\right)$ for all $i$.

\section{THE RADICAL SUBGROUPS}

In this section we shall give a description of the radical subgroups of classical groups. We first consider the unitary group $G=\mathrm{U}(n, q)$.

For integers $\alpha \geq 0$ and $\gamma \geq 0$, let $Z_{\alpha}$ be a cyclic group of order $r^{a+\alpha}, E_{\gamma}$ an extraspecial group of order $r^{2 \gamma+1}$, and $Z_{\alpha} E_{\gamma}$ a central product over $\Omega_{1}\left(Z_{\alpha}\right)=$ $Z\left(E_{\gamma}\right)$. By (1A) $Z_{\alpha} E_{\gamma}$ can be embedded as a subgroup of $\mathrm{GL}\left(r^{\gamma}, \varepsilon q^{e r^{\alpha}}\right)$ such that $Z_{\alpha}$ is identified with $O_{r}\left(Z\left(\operatorname{GL}\left(r^{\gamma}, \varepsilon q^{e r^{\alpha}}\right)\right)\right)$. Let $\Lambda_{\alpha}$ be a polynomial in $\mathscr{F}$ having a primitive $r^{a+\alpha}$ th root of unity as a root. The degree of $\Lambda_{\alpha}$ is $e r^{\alpha}$ (cf. $\left[11\right.$, p. 126]), so that $\mathrm{U}\left(e r^{\alpha+\gamma}, q\right)$ has a primary element $g$ with $\Lambda_{\alpha}$ as a unique elementary divisor of multiplicity $r^{\gamma}$. By (1.10)

$$
C(g) \simeq \mathrm{GL}\left(r^{\gamma}, \varepsilon q^{e r^{\alpha}}\right) \text {. }
$$

We may identify $\operatorname{GL}\left(r^{\gamma}, \varepsilon q^{e r^{\alpha}}\right)$ with $C(g)$, so that $\operatorname{GL}\left(r^{\gamma}, \varepsilon q^{e r^{\alpha}}\right)$ is embedded as a subgroup of $\mathrm{U}\left(e r^{\alpha+\gamma}, q\right)$ and $Z_{\alpha}=\langle g\rangle$. Let $R_{\alpha, \gamma}$ be the image of $Z_{\alpha} E_{\gamma}$ under the composition

$$
Z_{\alpha} E_{\gamma} \hookrightarrow \mathrm{GL}\left(r^{\gamma}, \varepsilon q^{e r^{\alpha}}\right) \hookrightarrow \mathrm{U}\left(e r^{\alpha+\gamma}, q\right)
$$

Since $Z_{\alpha}=\langle g\rangle$, a generator of $Z\left(R_{\alpha, \gamma}\right)$ is primary, so that by (1C) $R_{\alpha, \gamma}$ is uniquely determined by $Z_{\alpha} E_{\gamma}$ up to conjugacy. For integer $m \geq 1$, let $R_{m, \alpha, \gamma}$ be the image of the $m$-fold diagonal mapping of $R_{\alpha, \gamma}$ in $\mathrm{U}\left(m^{2} r^{\alpha+\gamma}, q\right)$ given by

$$
g \mapsto\left(\begin{array}{cccc}
g & & & \\
& g & & \\
& & \ddots & \\
& & & g
\end{array}\right), \quad g \in R_{\alpha, \gamma} .
$$

Then a generator of $Z\left(R_{m, \alpha, \gamma}\right)$ is the image of a generator of $Z\left(R_{\alpha, \gamma}\right)$ under the embedding above, so that it is primary in $\mathrm{U}\left(m e r^{\alpha+\gamma}, q\right)$ and then $R_{m, \alpha, \gamma}$ is uniquely determined by $m$ and $Z_{\alpha} E_{\gamma}$ up to conjugacy. Let $C_{m, \alpha, \gamma}$ and $N_{m, \alpha, \gamma}$ be the centralizer and normalizer of $R_{m, \alpha, \gamma}$ in $\mathrm{U}\left(m^{2} r^{\alpha+\gamma}, q\right)$, and let $N_{m, \alpha, \gamma}^{0}=\left\{g \in N_{m, \alpha, \gamma}:\left[g, Z\left(R_{m, \alpha, \gamma}\right)\right]=1\right\}$. By $(1 \mathrm{C}) C_{m, \alpha, \gamma} \simeq$ $\mathrm{GL}\left(m, \varepsilon q^{e r^{\alpha}}\right) \otimes I_{\gamma}$, where $I_{\gamma}$ is the identity matrix of order $r^{\gamma}$ and $\mathrm{GL}\left(m, \varepsilon q^{e r^{\alpha}}\right)$ $\otimes I_{\gamma}$ is the group $\left\{g \otimes I_{\gamma}: g \in \mathrm{GL}\left(m, \varepsilon q^{e r^{\alpha}}\right)\right\}$. If $R_{m, \alpha, \gamma}$ is radical, then $E_{\gamma}$ has exponent $r, N_{m, \alpha, \gamma}^{0}=L_{m, \alpha, \gamma} C_{m, \alpha, \gamma}$, and $N_{m, \alpha, \gamma} / N_{m, \alpha, \gamma}^{0}$ is cyclic of order $e r^{\alpha}$, where $L_{m, \alpha, \gamma}$ is a subgroup of $N_{m, \alpha, \gamma}^{0}$ containing $R_{m, \alpha, \gamma}$ such that $L_{m, \alpha, \gamma} \cap C_{m, \alpha, \gamma}=Z\left(L_{m, \alpha, \gamma}\right)=Z\left(C_{m, \alpha, \gamma}\right),\left[L_{m, \alpha, \gamma}, C_{m, \alpha, \gamma}\right]=1$, and $L_{m, \alpha, \gamma} / Z\left(L_{m, \alpha, \gamma}\right) R_{m, \alpha, \gamma} \simeq \operatorname{Sp}(2 \gamma, r)$. In particular, $R_{m, \alpha, \gamma}$ is uniquely determined by $m, \alpha$, and $\gamma$ up to conjugacy. Moreover, each linear character of $Z\left(L_{m, \alpha, \gamma}\right)$ acting trivially on $O_{r}\left(Z\left(L_{m, \alpha, \gamma}\right)\right)$ can be extended as a character of $L_{m, \alpha, \gamma}$ trivial on $R_{m, \alpha, \gamma}$. 
For integer $c \geq 1$, let $A_{c}$ denote the elementary abelian $r$-subgroup of order $r^{c}$ represented by its regular permutation representation. For any sequence $\mathbf{c}=\left(c_{1}, c_{2}, \ldots, c_{l}\right)$ of nonnegative integers, let $A_{\mathbf{c}}=A_{c_{1}} \mid A_{c_{2}}\left\{\cdots \mid A_{c_{l}}\right.$, and let

$$
R_{m, \alpha, \gamma, \mathrm{c}}=R_{m, \alpha, \gamma} \backslash A_{\mathrm{c}}
$$

be the wreath product in $\mathrm{U}(d, q)$, where $d=m e r^{\alpha+\gamma+c_{1}+\cdots+c_{l}}$. Then $R_{m, \alpha, \gamma, \mathrm{c}}$ is determined up to conjugacy in $\mathrm{U}(d, q)$. By [3, (1.4)], which applies to $\mathrm{U}(d, q)$ with some obvious modifications,

$$
C_{\mathrm{U}(d, q)}\left(R_{m, \alpha, \gamma, \mathrm{c}}\right)=C_{m, \alpha, \gamma} \otimes I_{\mathfrak{c}},
$$

where $I_{\mathbf{c}}$ is the identity matrix of order $u=r^{c_{1}+c_{2}+\cdots+c_{l}}$ and $C_{m, \alpha, \gamma} \otimes I_{\mathbf{c}}$ is defined as before. Moreover,

$$
\begin{aligned}
(2.2) \quad N_{\mathrm{U}(d, q)}\left(R_{m, \alpha, \gamma, \mathbf{c}}\right) & =\left(N_{m, \alpha, \gamma} / R_{m, \alpha, \gamma}\right) \otimes N_{\mathbf{S}(u)}\left(A_{\mathbf{c}}\right), \\
N_{\mathrm{U}(d, q)}\left(R_{m, \alpha, \gamma, \mathbf{c})} / R_{m, \alpha, \gamma, \mathbf{c}}\right. & \simeq\left(N_{m, \alpha, \gamma} / R_{m, \alpha, \gamma}\right) \times \mathrm{GL}\left(c_{1}, r\right) \times \cdots \times \mathrm{GL}\left(c_{l}, r\right),
\end{aligned}
$$

where $\left(N_{m, \alpha, \gamma} / R_{m, \alpha, \gamma}\right) \otimes N_{\mathrm{S}(u)}\left(A_{\mathrm{c}}\right)$ is defined as [3, (1.5)]. The proof of (2.2) is the same as that of $[3,(4.1)]$ with GL replaced by $U$ and some obvious modifications. We shall call $R_{m, \alpha, \gamma, \mathrm{c}}$ a basic subgroup of $\mathrm{U}(d, q), d$ the degree $d\left(R_{m, \alpha, \gamma, \mathrm{c}}\right)$ of $R_{m, \alpha, \gamma, \mathrm{c}}$, and $l$ the length $l\left(R_{m, \alpha, \gamma, \mathrm{c}}\right)$ of $R_{m, \alpha, \gamma, \mathrm{c}}$.

Let $V$ be a unitary space over $\mathbb{F}_{q^{2}}$, or a symplectic or orthogonal space over $\mathbb{F}_{q}$ with type $\eta= \pm 1$ if $V$ is orthogonal. Let $G=\mathrm{U}(V)$ or $I(V)$, and let $R$ be an $r$-subgroup of $G$. We shall say that an $R$-submodule $W$ of $V$ is nondegenerate or totally isotropic if $W$ is respectively a nondegenerate or a totally isotropic subspace of $V$.

(2A). Let $R$ be an $r$-subgroup of $G$. Then $V$ has an $R$-module decomposition

$$
V=V_{1} \perp V_{2} \perp \cdots \perp V_{v} \perp\left(U_{v+1} \oplus U_{v+1}^{\prime}\right) \perp \cdots \perp\left(U_{w} \oplus U_{w}^{\prime}\right),
$$

where the $V_{i}$ for $1 \leq i \leq v$ are nondegenerate simple $R$-submodules, the $U_{j}$ and $U_{j}^{\prime}$ for $v+1 \leq j \leq w$ are totally isotropic simple $R$-submodules such that $U_{j} \oplus U_{j}^{\prime}$ is nondegenerate and has no proper nondegenerate $R$-submodule. Moreover, if $R$ is abelian and the set of vectors $[V, R]$ moved by $R$ is $V$, then $v=0$ or $v=w$ according as $\varepsilon=1$ or -1 .

Proof. The first half of (2A) follows by the proof of [5, (1B)]. Suppose $R$ is abelian and $[V, R]=V$. Let $\mathbf{F}_{i}$ be the representation of $R$ on $V_{i}$ or $U_{i} \oplus U_{i}^{\prime}$ according as $i \leq v$ or $i \geq v+1$. If $i \leq v$, then $V_{i}$ is a simple $R$-module and the commuting algebra $D$ of $R$ on $V_{i}$ contains $\mathbf{F}_{i}(R)$. If $i \geq v+1$, then $U_{i}$ is a simple $R$-module and the representation of $R$ on $U_{i}^{\prime}$ is the contragredient of the representation $\mathbf{W}$ of $R$ on $U_{i}$ composed with a field automorphism. Thus the commuting algebra $D$ of $R$ on $U_{i}$ contains $\mathbf{W}(R)$. Since $D$ is a field and $D^{\times}=D \backslash\{0\}$ is a cyclic group, $\mathbf{F}_{i}(R)$ is cyclic generated by $g_{i}$ for some $g_{i} \in I\left(V_{i}\right)$ or $I\left(U_{i} \oplus U_{i}^{\prime}\right)$ according as $i \leq v$ or $i \geq v+1$, so that $V_{i}$ or $U_{i}$ is a simple $\left\langle g_{i}\right\rangle$-module. By $(1.8) g_{i}$ is primary with a unique elementary divisor $\Gamma \in \mathscr{F}_{1} \cup \mathscr{F}_{2}$ of multiplicity 1 . Since $g_{i}$ is an $r$-element, it follows that $\Gamma \in \mathscr{F}_{1}$ or $\mathscr{F}_{2}$ according as $\varepsilon=-1$ or 1 . Thus the underlying space of $\mathrm{F}_{i}(R)$ has the form $V_{i}$ or $U_{i} \oplus U_{i}^{\prime}$ according as $\varepsilon=-1$ or 1 . This proves $(2 \mathrm{~A})$. 
(2B). Let $G=\mathrm{U}(V), R$ a radical $r$-subgroup of $G$, and $N=N_{G}(R)$. Then there exists a corresponding decomposition

$$
V=V_{0} \perp V_{1} \perp \cdots \perp V_{t}, \quad R=R_{0} \times R_{1} \times \cdots \times R_{t}
$$

such that $R_{0}$ is the trivial subgroup of $\mathrm{U}\left(V_{0}\right)$ and $R_{i}$ is a basic subgroup of $\mathrm{U}\left(V_{i}\right)$ for $i \geq 1$. Moreover, the extraspecial components of $R_{i}$ for $i \geq 1$ have exponent $r$.

Proof. Let $V_{0}=C_{V}(R)$ be the set of vectors in $V$ fixed by each element of $R$ and $V_{+}=[V, R]$. Then $V=V_{0} \perp V_{+}$and $R=R_{0} \times R_{+}$, where $R_{0}=\left\langle 1_{V_{0}}\right\rangle$ and $R_{+} \leq \mathrm{U}\left(V_{+}\right)$. So $N=\mathrm{U}\left(V_{0}\right) \times N_{\mathrm{U}\left(V_{+}\right)}\left(R_{+}\right)$and $R_{+}$is necessarily radical in $\mathrm{U}\left(V_{+}\right)$. We may suppose $V=V_{+}$by induction. Let $\mathrm{F}$ be the natural representation of $R$ in $G$. The same proof with some obvious modifications as that of [5, (2B)] shows that $R$ can be reduced to the following case: Every characteristic abelian subgroup of $R$ is cyclic and $V=w V_{1}$ for some $w \geq 1$ such that either $V_{1}$ is a nondegenerate simple $R$-module or $V_{1}$ decomposes as $U_{1} \otimes U_{1}^{\prime}$, where $U_{1}$ and $U_{1}^{\prime}$ are totally isotropic simple $R$-modules and $V_{1}$ has no proper nondegenerate $R$-submodule. In particular, $Z(F(R))$ is cyclic.

By a result of Hall, [14, 5.4.9], $R$ is a group $Z E$ of symplectic type, where $Z$ is a cyclic $r$-group and $E$ is an extraspecial $r$-group of order $r^{2 \gamma+1}$. Thus $Z(\mathbf{F}(R))=\mathbf{F}(Z)$ and we may suppose $\mathbf{F}(Z)=\langle z\rangle$. Let $H=C_{G}(\mathbf{F}(Z))$ and $C=C_{G}(\mathbf{F}(R))$. Then $\mathbf{F}(R) \leq H$ and $C \leq H$, so $Z(H) \unlhd C$. Since $\mathbf{F}(R)$ is radical in $G$ and $C \unlhd N$, it follows $O_{r}(C) \leq Z(\mathbf{F}(R))$, so that $O_{r}(Z(H)) \leq$ $O_{r}(C) \leq Z(\mathbf{F}(R))$ and $O_{r}\left(Z\left(C_{G}(z)\right)\right) \leq \mathbf{F}(Z)$. Thus $O_{r}\left(Z\left(C_{G}(z)\right)\right)$ is cyclic and by $(1.9)$ and $(1.10) z$ is primary with a unique elementary divisor $\Gamma \in$ $\mathscr{F}$. So $H \simeq \mathrm{GL}\left(m_{\Gamma}(z), \varepsilon q^{\delta_{\Gamma}}\right)$. Identify $H$ with $\mathrm{GL}\left(m_{\Gamma}(z), \varepsilon q^{\delta_{\Gamma}}\right)$. Then a generator of $\mathrm{F}(Z(E))$ is represented by a scalar multiple of the identity matrix, so that $m_{\Gamma}(z)=m r^{\gamma}$ for some integer $m \geq 1$ by Remark (1) of (1A). Since $O_{r}(Z(H)) \leq \mathbf{F}(Z)$ and $z \in O_{r}(Z(H)), \mathbf{F}(Z)=O_{r}(Z(H))$, so that $|Z|=r^{a+\alpha}$ for some integer $\alpha \geq 0$. By (1C) $R=R_{m, \alpha, \gamma}$ and $E$ has exponent $r$. This proves $(2 \mathrm{~B})$.

Let $(R, \varphi)$ be a weight of $G=\mathrm{U}(V)$ and let

$$
V=V_{0} \perp V_{1} \perp \cdots \perp V_{t}, \quad R=R_{0} \times R_{1} \times \cdots \times R_{t}
$$

be the corresponding decomposition of (2B). We define

$$
V(m, \alpha, \gamma, \mathrm{c})=\sum_{i} V_{i}, \quad R(m, \alpha, \gamma, \mathrm{c})=\prod_{i} R_{i},
$$

where $i$ runs over all indices such that $R_{i}=R_{m, \alpha, \gamma, \mathrm{c}}$.

(2C). With the preceding notation

$$
\begin{aligned}
N(R) & =\mathrm{U}\left(V_{0}\right) \times \prod_{m, \alpha, \gamma, \mathbf{c}} N_{\mathrm{U}(V(m, \alpha, \gamma, \mathbf{c}))}(R(m, \alpha, \gamma, \mathbf{c})), \\
N(R) / R & =\mathrm{U}\left(V_{0}\right) \times \prod_{m, \alpha, \gamma, \mathbf{c}} N_{\mathrm{U}(V(m, \alpha, \gamma, \mathbf{c}))}(R(m, \alpha, \gamma, \mathbf{c})) / R(m, \alpha, \gamma, \mathbf{c}) .
\end{aligned}
$$

Moreover,

$$
\begin{aligned}
& N_{\mathrm{U}(V(m, \alpha, \gamma, \mathbf{c}))}(R(m, \alpha, \gamma, \mathbf{c}))=N_{\mathrm{U}\left(V_{m, \alpha, \gamma, \mathrm{c}}\right)}\left(R_{m, \alpha, \gamma, \mathbf{c}}\right) \imath \mathbf{S}(u), \\
& N_{\mathrm{U}(V(m, \alpha, \gamma, \mathbf{c}))}(R(m, \alpha, \gamma, \mathbf{c})) / R(m, \alpha, \gamma, \mathbf{c}) \\
& \quad=\left(N_{\mathrm{U}\left(V_{m, \alpha, \gamma, \mathrm{c}}\right)}\left(R_{m, \alpha, \gamma, \mathbf{c}}\right) / R_{m, \alpha, \gamma, \mathbf{c}}\right) \imath \mathbf{S}(u)
\end{aligned}
$$


where $V_{m, \alpha, \gamma, \mathrm{c}}$ is the underlying space of $R_{m, \alpha, \gamma, \mathrm{c}}$ and $u$ is the number of basic components $R_{m, \alpha, \gamma, \mathrm{c}}$ in $R(m, \alpha, \gamma, \mathrm{c})$.

Proof. The proof of [3, (4B)] can be applied here with GL replaced by $U$ and some obvious modifications.

We now consider radical subgroups of classical groups and as before, we suppose $q$ is odd. For integers $\alpha \geq 0$ and $\gamma \geq 0$, let $\Lambda_{\alpha}$ be a polynomial in $\mathscr{F}$ having a primitive $r^{a+\alpha}$ th root of unity as a root. Then the degree of $\Lambda_{\alpha}$ is $2 e r^{\alpha}$ and $\Lambda_{\alpha} \in \mathscr{F}_{1}$ or $\mathscr{F}_{2}$ according as $\varepsilon=-1$ or 1 (see [12, (5.1)]). Let $V_{\alpha, \gamma}$ be a symplectic or orthogonal space over $\mathbb{F}_{q}$ of dimension $2 e r^{\alpha+\gamma}$ and $\eta\left(V_{\alpha, \gamma}\right)=\varepsilon$ if $V_{\alpha, \gamma}$ is orthogonal. Then by (1.11) $I\left(V_{\alpha, \gamma}\right)$ has a primary element $g$ with a unique elementary divisor $\Lambda_{\alpha}$ of multiplicity $r^{\gamma}$. By (1.10) $C_{I\left(V_{\alpha, \gamma}\right)}(g) \simeq \mathrm{GL}\left(r^{\gamma}, \varepsilon q^{e r^{\alpha}}\right)$ and we may identify these two groups. By (1A) $Z_{\alpha} E_{\gamma}$ can be embedded as a subgroup of $\mathrm{GL}\left(r^{\gamma}, \varepsilon q^{e r^{\alpha}}\right)$ such that $Z_{\alpha}=O_{r}\left(Z\left(\mathrm{GL}\left(r^{\gamma}, \varepsilon q^{e r^{\alpha}}\right)\right)\right)$, where $Z_{\alpha} E_{\gamma}$ is defined as before. The image $R_{\alpha, \gamma}$ of $Z_{\alpha} E_{\gamma}$ under the composition

$$
Z_{\alpha} E_{\gamma} \hookrightarrow \mathrm{GL}\left(r^{\gamma}, \varepsilon q^{e r^{\alpha}}\right) \hookrightarrow I\left(V_{\alpha, \gamma}\right)
$$

is then determined up to conjugacy. A generator of $Z\left(R_{\alpha, \gamma}\right)$ is primary, so by (1C) $R_{\alpha, \gamma}$ is uniquely determined by $Z_{\alpha} E_{\gamma}$ up to conjugacy.

For integer $m \geq 1$, let $V_{m, \alpha, \gamma}=V_{\alpha, \gamma} \perp V_{\alpha, \gamma} \perp \cdots \perp V_{\alpha, \gamma}$ ( $m$ terms), and let $R_{m, \alpha, \gamma}$ be the image of the $m$-fold diagonal mapping of $R_{\alpha, \gamma}$ in $I\left(V_{m, \alpha, \gamma}\right)$ given by

$$
g \mapsto\left(\begin{array}{cccc}
g & & & \\
& g & & \\
& & \ddots & \\
& & & g
\end{array}\right), \quad g \in R_{\alpha, \gamma} .
$$

The same proof as the unitary case shows that $R_{m, \alpha, \gamma}$ is also uniquely determined by $m$ and $Z_{\alpha} E_{\gamma}$ up to conjugacy. In addition, $\eta\left(V_{m, \alpha, \gamma}\right)=\varepsilon^{m}$ if $V_{m, \alpha, \gamma}$ is orthogonal.

Let $C_{m, \alpha, \gamma}$ and $N_{m, \alpha, \gamma}$ be the centralizer and normalizer of $R_{m, \alpha, \gamma}$ in $I\left(V_{m, \alpha, \gamma}\right)$ respectively, and let $N_{m, \alpha, \gamma}^{0}=\left\{g \in N_{m, \alpha, \gamma}:\left[g, Z\left(R_{m, \alpha, \gamma}\right)\right]=\right.$ 1\}. Then $N_{m, \alpha, \gamma}^{0} \unlhd N_{m, \alpha, \gamma}$ and by (1C) $C_{m, \alpha, \gamma} \simeq \mathrm{GL}\left(m, \varepsilon q^{e r^{\alpha}}\right) \otimes I_{\gamma}$, where $I_{\gamma}$ is the identity matrix of degree $r^{\gamma}$ and $\mathrm{GL}\left(m, \varepsilon q^{e r^{\alpha}}\right) \otimes I_{\gamma}$ is defined as in the unitary case. In particular, if $R_{m, \alpha, \gamma}$ is radical in $I\left(V_{m, \alpha, \gamma}\right)$, then $R_{m, \alpha, \gamma}$ has exponent $r, N_{m, \alpha, \gamma}^{0}=L_{m, \alpha, \gamma} C_{m, \alpha, \gamma}$, and $N_{m, \alpha, \gamma} / N_{m, \alpha, \gamma}^{0}$ is cyclic of order $2 e r^{\alpha}$, where $L_{m, \alpha, \gamma} \cap C_{m, \alpha, \gamma}=Z\left(L_{m, \alpha, \gamma}\right)=Z\left(C_{m, \alpha, \gamma}\right)$, $\left[L_{m, \alpha, \gamma}, C_{m, \alpha, \gamma}\right]=1, R_{m, \alpha, \gamma} \leq L_{m, \alpha, \gamma}$, and $L_{m, \alpha, \gamma} / Z\left(L_{m, \alpha, \gamma}\right) R_{m, \alpha, \gamma} \simeq$ $\mathrm{Sp}(2 \gamma, r)$. So $R_{m, \alpha, \gamma}$ is uniquely determined by $m, \alpha$, and $\gamma$ up to conjugacy in $I\left(V_{m, \alpha, \gamma}\right)$. Moreover, by (1C) each linear character of $Z\left(L_{m, \alpha, \gamma}\right)$ acting trivially on $O_{r}\left(Z\left(L_{m, \alpha, \gamma}\right)\right)$ can be extended as a character of $L_{m, \alpha, \gamma}$ acting trivially on $R_{m, \alpha, \gamma}$.

For each sequence $\mathbf{c}=\left(c_{1}, c_{2}, \ldots, c_{l}\right)$ of nonnegative integers, let

$$
\begin{aligned}
V_{m, \alpha, \gamma, \mathbf{c}} & =V_{m, \alpha, \gamma} \perp V_{m, \alpha, \gamma} \perp \cdots \perp V_{m, \alpha, \gamma} \quad(u \text { terms }), \\
A_{\mathbf{c}} & =A_{c_{1}} \backslash A_{\mathcal{C}_{2}} l \cdots \succ A_{\mathcal{C}_{l}}, \quad R_{m, \alpha, \gamma, \mathbf{c}}=R_{m, \alpha, \gamma} \backslash A_{\mathbf{c}},
\end{aligned}
$$

where $u=r^{c_{1}+c_{2}+\cdots+c_{l}}$ and each $A_{c_{i}}$ is defined as before. Then $R_{m, \alpha, \gamma, \mathrm{c}}$ is determined up to conjugacy in $I\left(V_{m, \alpha, \gamma, \mathbf{c}}\right)$ and $\eta\left(V_{m, \alpha, \gamma, \mathbf{c}}\right)=\varepsilon^{m}$ if $V_{m, \alpha, \gamma, \mathbf{c}}$ 
is orthogonal. By $[3,(1.4)]$ with some obvious modifications

$$
C_{I\left(V_{m, \alpha, \gamma, \mathrm{c}}\right)}\left(R_{m, \alpha, \gamma, \mathrm{c}}\right)=C_{m, \alpha, \gamma} \otimes I_{\mathbf{c}},
$$

where $I_{c}$ is the identity matrix of order $u$ and the right-hand sides is defined as before. Moreover, the same proof as that of $[3,(4.1)]$ with GL replaced by $I$ shows that

$$
\begin{aligned}
& N_{I\left(V_{m, \alpha, \gamma, \mathrm{c}}\right)}\left(R_{m, \alpha, \gamma, \mathrm{c}}\right)=\left(N_{m, \alpha, \gamma} / R_{m, \alpha, \gamma}\right) \otimes N_{\mathbf{S}(u)}\left(A_{\mathbf{c}}\right), \\
& N_{I\left(V_{m, \alpha, \gamma, \mathrm{c}}\right)}\left(R_{m, \alpha, \gamma, \mathbf{c}}\right) / R_{m, \alpha, \gamma, \mathbf{c}} \\
& \quad=\left(N_{m, \alpha, \gamma} / R_{m, \alpha, \gamma}\right) \times \mathrm{GL}\left(c_{1}, r\right) \times \cdots \times \mathrm{GL}\left(c_{l}, r\right),
\end{aligned}
$$

where $\left(N_{m, \alpha, \gamma} / R_{m, \alpha, \gamma}\right) \otimes N_{\mathbf{S}(u)}\left(A_{\mathbf{c}}\right)$ is defined as [3, (1.5)]. We shall call $R_{m, \alpha, \gamma, \mathrm{c}}$ a basic subgroup of $I\left(V_{m, \alpha, \gamma, \mathrm{c}}\right), \operatorname{dim} V_{m, \alpha, \gamma, \mathrm{c}}$ the degree $d\left(R_{m, \alpha, \gamma, \mathrm{c}}\right)$ of $R_{m, \alpha, \gamma, \mathrm{c}}$, and $l$ the length $l\left(\boldsymbol{R}_{m, \alpha, \gamma, \mathrm{c}}\right)$ of $R_{m, \alpha, \gamma, \mathrm{c}}$.

(2D). Let $V$ be a symplectic or orthogonal space over $\mathbb{F}_{q}, G=I(V)$ the group of all isometries of $V$, and $R$ a radical subgroup of $G$. Then there exists a corresponding decomposition

$$
V=V_{0} \perp V_{1} \perp \cdots \perp V_{t}, \quad R=R_{0} \times R_{1} \times \cdots \times R_{t},
$$

such that $R_{0}$ is the trivial subgroup of $I\left(V_{0}\right)$ and $R_{i}$ is a basic subgroup of $I\left(V_{i}\right)$ for $i \geq 1$. Moreover, the extraspecial components of $R_{i}$ for $i \geq 1$ have exponent $r$.

Proof. Let $V_{0}=C_{V}(R)$ and $V_{+}=[V, R]$. Then $V=V_{0} \perp V_{+}$and $R=$ $R_{0} \times R_{+}$, where $R_{0}=\left\langle 1_{V_{0}}\right\rangle$ and $R_{+} \leq I\left(V_{+}\right)$. In particular, $N(R)=I\left(V_{0}\right) \times$ $N_{I\left(V_{+}\right)}\left(R_{+}\right)$and $R_{+}$is necessarily a radical subgroup of $I\left(V_{+}\right)$. By induction we may suppose $V=V_{+}$. Thus $Z(R)$ is abelian and $[V, Z(R)]=V$. By $(2 \mathrm{~A})$ we may write the $Z(R)$-module $V$ as

$$
V=m_{1} V_{1} \perp m_{2} V_{2} \perp \cdots \perp m_{w} V_{w},
$$

where each $V_{i}$ is either a nondegenerate simple $Z(R)$-submodule or a sum $U_{i} \oplus U_{i}^{\prime}$ of totally isotropic simple $Z(R)$-submodules $U_{i}, U_{i}^{\prime}$ according as $\varepsilon=$ -1 or 1 , and $m_{i}$ is the multiplicity of $V_{i}$ in $V$ for all $i \geq 1$. If $\varepsilon=-1$, then $r \mid q^{e}+1$ and $\mathbb{F}_{q^{2 e} e_{i} a_{i}}$ is the commuting algebra of $Z(R)$ on $V_{i}$ for some $\alpha_{i} \geq 0$ since $\left[V_{i}, Z(R)\right]=V_{i}$ and $Z(R)$ is an $r$-group. Similarly, if $\varepsilon=1$, then $r \mid q^{e}-1, V_{i}=U_{i} \oplus U_{i}^{\prime}$, and $\mathbb{F}_{q^{e} r_{i}}$ is the commuting algebra of $Z(R)$ on $U_{i}$ for some integer $\alpha_{i} \geq 0$. In all cases $\operatorname{dim} V_{i}=2 e r^{\alpha_{i}}$. Let $N^{0}=\{g \in$ $N(R):[g, Z(R)]=1\}$, and let $H=C_{G}(Z(R))$. Then $h\left(m_{i} V_{i}\right)=m_{i} V_{i}$ for $h \in H$ and all $i \geq 1$. Thus there exists a corresponding decomposition

$$
H=H_{1} \times H_{2} \times \cdots \times H_{w}
$$

such that $H_{i} \simeq \mathrm{GL}\left(m_{i}, \varepsilon q^{e r^{\alpha_{i}}}\right) \leq I\left(m_{i} V_{i}\right)$ for all $i \geq 1$. Since $R$ is radical and $N^{0} \unlhd N$, it follows $O_{r}\left(N^{0}\right) \leq O_{r}(N)=R$. On the other hand, $R \leq N^{0}$ and $N^{0}=N_{H}(R)$, so $R=O_{r}\left(N^{0}\right)$ and $R$ is radical in $H$.

Let $R_{i}$ be the group of linear operators which agree with an element of $R$ on $m_{i} V_{i}$ and are the identity on $m_{j} V_{j}$ for $j \neq i$. Then $N^{0}$ permutes the pairs $\left(m_{i} V_{i}, R_{i}\right)$ for $1 \leq i \leq w$, so that $R \leq N^{0} \cap R_{1} \times R_{2} \times \cdots \times R_{w} \unlhd N^{0}$. It follows that $R=R_{1} \times R_{2} \times \cdots \times R_{w}$ and $R_{i}=O_{r}\left(N_{i}\right)$, where $N_{i}=N_{H_{i}}\left(R_{i}\right)$. Thus $R_{i}$ is radical in $H_{i}$ for all $i$. By induction on $\operatorname{dim} V$, we may suppose $w=1$, so that $V=m_{1} V_{1}, R=R_{1}, H=H_{1}$, and $Z(R)=Z\left(R_{1}\right)$ is cyclic generated by some 
$x \in I(V)$. But $H=C_{G}(x)$ and $O_{r}(Z(H)) \leq O_{r}(H)$, so $O_{r}(Z(H)) \leq Z(R)$. By (1.9) and (1.10) $x$ is primary in $G$. Apply [3, (4A)] or (2B) to $H \simeq$ $\mathrm{GL}\left(m_{1}, \varepsilon q^{e^{\alpha_{1}}}\right)$. So $R$ is a basic subgroup $R_{m, \alpha, \gamma, \mathrm{c}}$ of $H$, where $m, \gamma, \alpha$ are integers, and $\mathbf{c}=\left(c_{1}, \ldots, c_{l}\right)$ is a sequence of nonnegative integers such that $\alpha \geq \alpha_{1}$, and $m e r^{\alpha+\gamma+c_{1}+\cdots+c_{l}}=m_{1} e r^{\alpha_{1}}$. Moreover, the extraspecial components of $R_{m, \alpha, \gamma, c}$ have exponent $r$. In particular, $\operatorname{dim} V=2 m e r^{\alpha+\gamma+c_{1}+\cdots+c_{l}}$ and $\eta(V)=\varepsilon^{m}=\varepsilon^{m_{1}}$ if $V$ is orthogonal. Thus $I(V)$ has a basic subgroup $R^{\prime}$ of the form $R_{m, \alpha, \gamma, c}$ defined by (2.4), where the extraspecial components of $R^{\prime}$ have exponent $r$. So $Z(R)$ and $Z\left(R^{\prime}\right)$ are cyclic generated by primary elements of order $r^{a+\alpha}$ in $I(V)$, and they are conjugate in $I(V)$. Thus we may suppose $Z(R)=Z\left(R^{\prime}\right)$, so that $R^{\prime} \leq H$. By definition $R^{\prime}$ still has the type $R_{m, \alpha, \gamma, \mathrm{c}}$ as a subgroup of $H$, so that $R^{\prime}$ and $R$ are conjugate in $H$. Thus $R=R_{m, \alpha, \gamma, \mathrm{c}}$ is a basic subgroup of $I(V)$ and (2D) follows.

Remark. In the notation of (2B) or (2D), suppose $t \neq 0$. Then there exists an element $\rho$ of $Z(R)$ such that (1) $|\rho|=r^{a} ;(2)[V, \rho]=\sum_{i=1}^{t} V_{i} ;(3)$ the restriction of $\rho$ on $[V, \rho]$ is primary. Such an element exists by $(2 B)$ or (2D) and will be called a primary element of $R$. If $\rho$ is a primary element of $R$, then $\langle\rho\rangle$ is uniquely determined by $R$ up to conjugacy and $C_{G}(\rho) \simeq$ $\mathrm{U}\left(V_{0}\right) \times \mathrm{GL}\left(m, \varepsilon q^{e}\right)$ or $C_{G}(\rho) \simeq I\left(V_{0}\right) \times \mathrm{GL}\left(m, \varepsilon q^{e}\right)$ for some $m \geq 1$ according as $G=\mathrm{U}(V)$ or $I(V)$.

Let $(R, \varphi)$ be a weight of $G=I(V)$ and let

$$
V=V_{0} \perp V_{1} \perp \cdots \perp V_{t}, \quad R=R_{0} \times R_{1} \times \cdots \times R_{t},
$$

be the corresponding decomposition of (2D). We define

$$
V(m, \alpha, \gamma, \mathbf{c})=\sum_{i} V_{i}, \quad R(m, \alpha, \gamma, \mathbf{c})=\prod_{i} R_{i},
$$

where $i$ runs over all indices such that $R_{i}=R_{m, \alpha, \gamma, \mathrm{c}}$.

(2E). With the preceding notation

$$
\begin{aligned}
N(R) & =I\left(V_{0}\right) \times \prod_{m, \alpha, \gamma, \mathrm{c}} N_{I(V(m, \alpha, \gamma, \mathbf{c}))}(R(m, \alpha, \gamma, \mathrm{c})), \\
N(R) / R & =I\left(V_{0}\right) \times \prod_{m, \alpha, \gamma, \mathbf{c}} N_{I(V(m, \alpha, \gamma, \mathbf{c}))}(R(m, \alpha, \gamma, \mathbf{c})) / R(m, \alpha, \gamma, \mathbf{c}) .
\end{aligned}
$$

Moreover,

$$
\begin{aligned}
& N_{I(V(m, \alpha, \gamma, \mathbf{c}))}(R(m, \alpha, \gamma, \mathbf{c}))=N_{I\left(V_{m, \alpha, \gamma, \mathrm{c}}\right.}\left(R_{m, \alpha, \gamma, \mathrm{c}}\right) l \mathbf{S}(u), \\
& N_{I(V(m, \alpha, \gamma, \mathrm{c}))}(R(m, \alpha, \gamma, \mathbf{c})) / R(m, \alpha, \gamma, \mathbf{c}) \\
& \quad=\left(N_{I\left(V_{m, \alpha, \gamma, \mathrm{c}}\right)}\left(R_{m, \alpha, \gamma, \mathrm{c}}\right) / R_{m, \alpha, \gamma, \mathrm{c}}\right) \mathbf{S}(u),
\end{aligned}
$$

where $V_{m, \alpha, \gamma, \mathrm{c}}$ is the underlying space of $R_{m, \alpha, \gamma, \mathrm{c}}$ and $u$ is the number of basic components $R_{m, \alpha, \gamma, \mathrm{c}}$ in $R(m, \alpha, \gamma, \mathrm{c})$.

Proof. The proof is essentially the same as that of [3, (4B)] with GL replaced by $I$ and some obvious modifications, except the minimal elements of $\mathscr{E}_{i}$ have dimension $2 m e r^{\alpha+\gamma}$ when $R_{i}=R_{m, \alpha, \gamma, \mathrm{c}}$, where $\mathscr{E}_{i}$ is defined there.

\section{MORE ON BASIC SUBGROUPS}

Let $R$ be a radical subgroup of a finite group $G, N=N(R), C=C(R)$. The stabilizer in $N$ of an irreducible character $\theta$ of $C R$ will be denoted by 
$N(\theta)$. We denote the sets of irreducible characters of $N(\theta)$ and $N$ which cover $\theta$ and which have defect 0 as characters of $N(\theta) / R$ and $N / R$ respectively by $\operatorname{Irr}^{0}(N(\theta), \theta)$ and $\operatorname{Irr}^{0}(N, \theta)$. By Clifford theory the induction mapping $\psi \mapsto I(\psi)=\operatorname{Ind}_{N(\theta)}^{N}(\psi)$ induces a bijection from $\operatorname{Irr}^{0}(N(\theta), \theta)$ to $\operatorname{Irr}^{0}(N, \theta)$. Since $\psi(1)=d(\psi) \theta(1)$ for some integral divisor $d(\psi)$ of $(N(\theta): C R)$, it follows that $(R, I(\psi))$ is a weight of $G$ if and only if

$$
d(\psi)_{r}=(N(\theta): C R)_{r}, \quad \theta(1)_{r}=(C R: R)_{r},
$$

and in particular, $\theta$ then has defect 0 as a character of $C R / R$. In this case the block $b$ of $C R$ containing $\theta$ has a defect group $R$ and the canonical character $\theta$. Moreover, for any $\psi$ of $\operatorname{Irr}^{0}(N(\theta), \theta), I(\psi)$ is a character of $b^{N}$ and $(R, I(\psi))$ is a $b^{G}$-weight of $G$. Following [3, p. 3] all $B$-weights for a block $B$ of $G$ have the form $(R, I(\psi))$, where $R$ runs over representatives for the conjugacy $G$-classes of radical subgroups, $b$ runs over representatives for the conjugacy $N(R)$-classes of blocks of $C(R) R$ such that $b$ has defect group $R$ and $b^{G}=B$, and $\psi$ runs over $\operatorname{Irr}^{0}(N(\theta), \theta)$. Here $\theta$ is the canonical character of $b$. A pair $(R, b)$ of an $r$-subgroup $R$ of $G$ and a block $b$ of $C$ is called a Brauer pair of $G$. In particular, pairs $(1, B)$ correspond to blocks $B$ of $G$.

Now we consider the unitary group $G=\mathrm{U}(n, q)$. Given $\Gamma \in \mathscr{F}$, let $e_{\Gamma}, \alpha_{\Gamma}, m_{\Gamma}$ be integers defined as follows: $e_{\Gamma}$ is the multiplicative order of $\varepsilon_{\Gamma} q^{d_{\Gamma}}$ modulo $r, r^{\alpha_{\Gamma}}=\left(d_{\Gamma}\right)_{r}$, and $m_{\Gamma} e^{\alpha_{\Gamma}}=d_{\Gamma} e_{\Gamma}$. By [7, (3.2)] the Brauer pairs $(R, b)$ of $G$ are labeled by ordered triples $(R, s, \kappa)$, where $s$ is a semisimple $r^{\prime}$-element of a dual group $G^{*}$ of $G$, and $\kappa=\prod_{\Gamma \in \mathscr{F}} \kappa_{\Gamma}$ is a product of partitions $\kappa_{\Gamma}$ such that each $\kappa_{\Gamma}$ is an $e_{\Gamma}$-core of a partition of the multiplicity $m_{\Gamma}(s)$ of $\Gamma$ in $s$. This labeling extends the labeling [11, (5D)] by Fong and Srinivasan for blocks $B$ of $G$ by ordered pairs $(s, \kappa)$. Since $G^{*} \simeq G$, we may identify $G^{*}$ with $G$.

Let $\mathscr{F}^{\prime}$ be the subset of $\mathscr{F}$ consisting of polynomials whose roots have $r^{\prime}$ order. In $[11,(5 \mathrm{~A})]$ each $\Gamma$ in $\mathscr{F}$ ' determines a block $B_{\Gamma}$ of $G_{\Gamma}=\mathrm{U}\left(e_{\Gamma} d_{\Gamma}, q\right)$ with defect group $R_{\Gamma}=R_{m_{\Gamma}, \alpha_{\Gamma}, 0}$ as follows: Let $C_{\Gamma}=C_{G_{\Gamma}}\left(R_{\Gamma}\right), N_{\Gamma}=$ $N_{G_{\Gamma}}\left(R_{\Gamma}\right)$, so that $C_{\Gamma} \simeq \mathrm{GL}\left(m_{\Gamma}, \varepsilon q^{e r_{\Gamma}{ }_{\Gamma}}\right)$ and $N_{\Gamma} / C_{\Gamma}$ is cyclic of order $e r^{\alpha_{\Gamma}}$. Then $C_{\Gamma}$ has a block $b_{\Gamma}$ with defect group $R_{\Gamma}$ and label $\left(s_{\Gamma},-\right)$ in $C_{\Gamma}^{*}$ such that as an element of $G_{\Gamma}^{*}, s_{\Gamma}$ is primary with a unique elementary divisor $\Gamma$ of multiplicity $e_{\Gamma}$. If $\theta_{\Gamma}$ is the canonical character of $b_{\Gamma}$ and $N\left(\theta_{\Gamma}\right)$ is its stabilizer in $N_{\Gamma}$, then $\left(N\left(\theta_{\Gamma}\right): C_{\Gamma}\right)=e_{\Gamma}$. The block $b_{\Gamma}$ induces a block $b_{\Gamma}^{G_{\Gamma}}$ of $G_{\Gamma}$ which will be denoted by $B_{\Gamma}$. Since $\left(e_{\Gamma}, r\right)=1, B_{\Gamma}$ has a defect group $R_{\Gamma}$ and the label $\left(s_{\Gamma},-\right)$ (see [7, 3.2]). We shall also write $s_{\Gamma}$ as $e_{\Gamma} \Gamma$. Conversely, let $G=\mathrm{U}\left(m e r^{\alpha}, q\right)$, and $B$ a block of $G$ with defect group $R=R_{m, \alpha, 0}$. By $[11,(4 \mathrm{~B})$ and $(5 \mathrm{~A})](m, r)=1$ and there exists a unique $\Gamma \in \mathscr{F}^{\prime}$ such that $\Gamma$ and $B$ correspond in the preceding manner. In particular, $m=m_{\Gamma}$ and $\alpha=\alpha_{\Gamma}$.

The proofs of the following two lemmas are similar to that of $[4,(3 \mathrm{~A})$ and (3B)].

(3A). Given $\Gamma \in \mathscr{F}^{\prime}$, let $G=\mathrm{U}\left(r^{\gamma} e_{\Gamma} d_{\Gamma}, q\right), R=R_{m_{\Gamma}, \alpha_{\Gamma}, \gamma}$ a basic subgroup of $G$, and $C=C_{G}(R)$. Then $C=C_{\Gamma} \otimes I_{\gamma}$, where $I_{\gamma}$ is the identity matrix of order $r^{\gamma}$. The irreducible character $\theta=\theta_{\Gamma} \otimes I_{\gamma}$ of $C$ defined by $\theta\left(c \otimes I_{\gamma}\right)=\theta_{\Gamma}(c)$ for $c \in C_{\Gamma}$ is then a character of defect 0 of $C R / R$, and $\left|\operatorname{Irr}^{0}(N(\theta), \theta)\right|=e_{\Gamma}$. 
Proof. All statements but the last are clear. Let $N=N_{G}(R)$, and $N^{0}$ the subgroup $\{g \in N:[g, Z(R)]=1\}$ of $N$. By $(1 \mathrm{C}) N^{0}=L C$ and $N / N^{0}$ is cyclic of order $e r^{\alpha_{\Gamma}}$, where $R \leq L, L \cap C=Z(L)=Z(C),[L, C]=1$, and $L / Z(L) R \simeq \operatorname{Sp}(2 \gamma, r)$. Moreover, each linear character of $Z(L)$ acting trivially on $O_{r}(Z(L))$ can be extended as a character of $L$ trivial on $R$. Thus $N^{0} \leq N(\theta)$, and $N(\theta) / N^{0}$ is cyclic. An irreducible constituent of the restriction of $\theta$ to $Z(C)$ is a linear character trivial on $O_{r}(Z(C))$ and so has an extension $\xi$ to $L$ trivial on $R$. Thus $\xi \theta$ is an extension of $\theta$ to $N^{0}$. Since $N^{0} / R C \simeq$ $L / Z(L) R \simeq \operatorname{Sp}(2 \gamma, r)$, the Steinberg character St of $N^{0} / R C$ can be regarded as a character of $N^{0}$ trivial on $C R$. Thus $\vartheta=\mathrm{St} \xi \theta$ is irreducible since its restriction to $C$ is irreducible. By (3.1) $\vartheta \in \operatorname{Irr}^{0}\left(N^{0}, \theta\right)$. Suppose $\psi$ is a character of $\operatorname{Irr}^{0}\left(N^{0}, \theta\right)$. Then by Clifford theory $\psi=\chi \xi \theta$ for some irreducible character $\chi$ of $N^{0}$ trivial on $C$. Since $\psi$ and $\xi \theta$ act trivially on $R, \chi$ acts trivially on $R$, so that $\chi$ is an irreducible character of $N^{0} / C R$. Since $\psi$ has defect 0 as a character of $N^{0} / R, \chi$ has defect 0 as a character of $N^{0} / R C \simeq \operatorname{Sp}(2 \gamma, r)$. Thus $\chi=\mathrm{St}$ and $\operatorname{Irr}^{0}\left(N^{0}, \theta\right)=\{\vartheta\}$. If $N(\vartheta)$ is the stabilizer of $\vartheta$ in $N$, then $N(\theta)=N(\vartheta)$ and $\operatorname{Irr}^{0}(N(\theta), \theta)=\operatorname{Irr}^{0}(N(\vartheta), \vartheta)$.

By (1C) a generator $\sigma$ of $N / N^{0}$ induces a field automorphism of order $e r^{\alpha_{\Gamma}}$ on $C(Z(R)) \simeq \mathrm{GL}\left(m_{\Gamma} r^{\gamma}, \varepsilon q^{e r_{\Gamma}}\right)$. Since $C=C_{\Gamma} \otimes I_{\gamma}$ is a subgroup of $C(Z(R))$ invariant under $\sigma, \sigma$ also induces a field automorphism of order $e r^{\alpha_{\Gamma}}$ on $C$. But a generator $\sigma_{1}$ of $N_{\Gamma} / C_{\Gamma}$ also induces a field automorphism of order $e r^{\alpha_{T}}$ on $C_{\Gamma} \simeq \mathrm{GL}\left(m_{\Gamma}, \varepsilon q^{e^{r_{\Gamma}}}\right)$. By replacing generators, we may suppose $\sigma$ induces $\sigma_{1}$ on $C_{\Gamma}$. It follows that $N(\theta) / N^{0} \simeq N\left(\theta_{\Gamma}\right) / C_{\Gamma}$ and $\left|N(\theta) / N^{0}\right|=\left|N\left(\theta_{\Gamma}\right) / C_{\Gamma}\right|=e_{\Gamma}$. Since $N(\theta) / N^{0}$ is cyclic, $\vartheta$ extends in $e_{\Gamma}$ ways to irreducible characters of $N(\vartheta)$ which cover $\vartheta$, and since $e_{\Gamma}$ is prime to $r$, these extensions are in $\operatorname{Irr}^{0}(N(\vartheta), \vartheta)$.

Remark. The weights $(R, I(\psi))$ of $G$ for $\psi \in \operatorname{Irr}^{0}(N(\theta), \theta)$ are $B$-weights, where $B$ is the block of $G$ labeled by $\left(r^{\gamma} e_{\Gamma} \Gamma,-\right), I$ is the induction operator from $N(\theta)$ to $N$, and $r^{\gamma} e_{\Gamma} \Gamma$ represents an element of $\mathrm{U}\left(r^{\gamma} e_{\Gamma} d_{\Gamma}, q\right)$ with a unique elementary divisor $\Gamma$ of multiplicity $r^{\gamma} e_{\Gamma}$. Indeed, if $b$ is the block of $C$ containing $\theta$, then $(R, b)$ is labeled by $\left(R, r^{\gamma} e_{\Gamma} \Gamma,-\right)$ and the weights are $b^{G}$-weights. Moreover, by $[7,3.2] b^{G}$ is labeled by $\left(r^{\gamma} e_{\Gamma} \Gamma,-\right)$.

Given $\Gamma \in \mathscr{F}^{\prime}$, let $G=\mathrm{U}\left(r^{d} e_{\Gamma} d_{\Gamma}, q\right)$ and $R=R_{m_{\Gamma}, \alpha_{\Gamma}, \gamma, \mathrm{c}}$ a basic subgroup of $G$, where $d$ and $\gamma$ are nonnegative integers, $\mathbf{c}=\left(c_{1}, c_{2}, \ldots, c_{l}\right)$ such that $\gamma+c_{1}+c_{2}+\cdots+c_{l}=d$. Then $C=C_{G}(R)=C_{\Gamma} \otimes I_{\gamma} \otimes I_{\mathbf{c}}$, where $I_{\gamma}, I_{\mathrm{c}}$ are identity matrices of orders $r^{\gamma}$ and $r^{c_{1}+c_{2}+\cdots+c_{l}}$ respectively. The irreducible character of $C$ defined by

$$
\theta\left(c \otimes I_{\gamma} \otimes I_{\mathbf{c}}\right)=\theta_{\Gamma}(c)
$$

for $c \in C_{\Gamma}$ is then a character of defect 0 of $C R / R$. We shall say the pair $(R, \theta)$ is of type $\Gamma$. If $b$ is the block of $C$ containing $\theta$, then $(R, b)$ has label $\left(R, r^{d} e_{\Gamma} \Gamma,-\right)$.

(3B). Let $G=\mathrm{U}(n, q), R$ a basic subgroup of $G, b$ a block of $C(R) R$ with defect group $R$, and $\theta$ the canonical character of $b$. Then $(R, \theta)$ has type $\Gamma$ for some $\Gamma \in \mathscr{F}^{\prime}$.

Proof. Suppose $R=R_{m, \alpha, \gamma, \mathrm{c}}$. Set $G_{1}=\mathrm{U}\left(m e r^{\alpha}, q\right), R_{1}=R_{m, \alpha, 0}, C_{1}=$ $C_{G_{1}}\left(R_{1}\right)$. So $C_{1} \simeq \mathrm{GL}\left(m, \varepsilon q^{e^{\alpha}}\right)$ and $C=C_{1} \otimes I_{\gamma} \otimes I_{\mathrm{c}}$. Then $\theta$ has the form 
$\theta_{1} \otimes I_{\gamma} \otimes I_{\mathbf{c}}$, where $\theta_{1}$ is a character of $C_{1}$. Since $\theta$ has defect 0 as a character of $C R / R$ and $C R / R \simeq C_{1} / R_{1}, \theta_{1}$ also has defect 0 as a character of $C_{1} / R_{1}$. The block $b_{1}$ of $C_{1}$ containing $\theta_{1}$ then has defect group $R_{1}$. By [11, (5A)] there is a unique $\Gamma \in \mathscr{F}^{\prime}$ such that $R_{1}=R_{\Gamma}$ and $\theta_{1}=\theta_{\Gamma}$. Thus $m=m_{\Gamma}$, $\alpha=\alpha_{\Gamma}$, and $(R, \theta)$ has type $\Gamma$.

Following the notation of [12], we denote $V$ and $V^{*}$ finite-dimensional symplectic or orthogonal spaces over $\mathbb{F}_{q}$ related as follows:

$\begin{array}{cccc}V & \operatorname{dim} V & V^{*} & \operatorname{dim} V^{*} \\ \text { symplectic } & 2 n & \text { orthogonal } & 2 n+1 \\ \text { orthogonal } & 2 n+1 & \text { symplectic } & 2 n \\ \text { orthogonal } & 2 n & \text { orthogonal } & 2 n\end{array}$

where $\eta(V)=\eta\left(V^{*}\right)=1$ in the first two cases and $\eta(V)=\eta\left(V^{*}\right)$ in the third case. Here $\eta(V)=1$ for a symplectic space as before. Moreover, $I(V)$ and $I\left(V^{*}\right)$ are the groups of isometries of $V$ and $V^{*}, I_{0}(V)$ and $I_{0}\left(V^{*}\right)$ the subgroups of $I(V)$ and $I\left(V^{*}\right)$ of determinant 1 . We shall call $I_{0}\left(V^{*}\right)$ the dual group of $I_{0}(V)$. Let $G=I_{0}(V)$ and $G^{*}=I_{0}\left(V^{*}\right)$. Given a semisimple element $s$ of $G^{*}$, let $(s)$ be the conjugacy class of $s$ in $G^{*}$, and let $\mathscr{E}(G,(s))$ be defined by [8, p. 57]. Namely $\mathscr{E}(G,(s))$ is the set of the irreducible constituents of Deligne-Lusztig generalized characters associated with $(s)$. Given a semisimple $r^{\prime}$-element $s$ of $G^{*}$, let

$$
\mathscr{E}_{r}(G,(s))=\bigcup_{u} \mathscr{E}(G,(s u)),
$$

where $u$ runs over all the $r$-elements of $C_{G^{*}}(s)$. By $[8,2.2], \mathscr{E}_{r}(G,(s))$ is a union of $r$-blocks.

The following lemma is due to Fong and Olsson.

(3C). Let $\rho$ be an r-element of $G, b$ a block of $H=C_{G}(\rho)$, and $B$ a block of $G$. Suppose $H$ is regular subgroup of $G, B \subseteq \mathscr{E}_{r}(G,(s))$, and $b \subseteq \mathscr{E}_{r}(H,(t))$. If $b^{G}=B$, then $s$ and $t$ are conjugate in $G^{*}$.

Proof. By Brauer's Second Main Theorem there exists a nonzero generalized decomposition number $d_{\chi, \varphi}^{\rho}$ for some irreducible character $\chi \in B$ and irreducible modular character $\varphi \in b$. Let $\chi^{\left(b^{\prime}\right)}(\rho \tau)=\sum_{\varphi^{\prime} \in b^{\prime}} d_{\chi, \varphi^{\prime}}^{\rho} \varphi^{\prime}(\tau)$, where $b^{\prime}$ is a block of $H, \tau$ runs over the $r^{\prime}$-elements in $H$, and $\varphi^{\prime}$ runs over the irreducible modular characters in $b^{\prime}$. Then $\chi(\rho \tau)=\sum_{b^{\prime}} \chi^{\left(b^{\prime}\right)}(\rho \tau)$. On the other hand, by the theorem of Curtis type [9, (3.7)],

$$
\chi(\rho \tau)=\sum_{b^{\prime}} \sum_{\zeta \in b^{\prime}}\left(\chi, R_{H}^{G}(\zeta)\right) \zeta(\rho \tau),
$$

where $R_{H}^{G}(\zeta)$ is the generalized Deligne-Lusztig character, $b^{\prime}$ runs over blocks of $H$, and $\zeta$ runs over the irreducible characters of $b^{\prime}$. Since the $\zeta(\rho \tau)$ for $\zeta \in b^{\prime}$ are linear combinations of the Brauer characters $\varphi(\tau)$ for $\varphi \in b^{\prime}$ and the $\varphi$ are linear independent, it follows that

$$
\chi^{(b)}(\rho \tau)=\sum_{\zeta \in b}\left(\chi, R_{H}^{G}(\zeta)\right) \zeta(\rho \tau),
$$

and $\chi^{(b)}(\rho \tau) \neq 0$ for some $r^{\prime}$-element $\tau$. So $\left(\chi, R_{H}^{G}(\zeta)\right) \neq 0$ for some $\zeta \in b$. Suppose $\chi \in \mathscr{E}(G,(s u))$ and $\zeta \in \mathscr{E}(H,(t v))$, where $u$ is an $r$-element in 
$C_{G^{*}}(s), v$ is an $r$-element in $C_{H^{*}}(t)$. Then $s u$ and $t v$ are conjugate in $G^{*}$. Since $s$ and $t$ are the $r^{\prime}$-parts of $s u$ and $t v$ respectively, $s$ and $t$ are conjugate in $G^{*}$.

Let $R$ be a radical $r$-subgroup of $G, b$ a block of $C_{G}(R) R$ with defect group $R, V_{0}=C_{V}(R)$, and $V_{+}=[V, R]$. Then $b^{G}$ is well defined and $b^{G} \subseteq$ $\mathscr{E}_{r}(G,(s))$ for some $s \in G^{*}$. We shall give a decomposition of $s$ corresponding to the decomposition $V_{0} \perp V_{+}$of $V$ and give a label to the Brauer pair $(R, b)$ when $V=V_{+}$, where $b$ is regarded as a block of $C_{G}(R)$. Let $\rho$ be a primary element of $R$ given by the remark of (2D), and let $K=C_{G}(\rho)$. Then $K=$ $K_{0} \times K_{+}$, where $K_{0}=I_{0}\left(V_{0}\right)$ and $K_{+} \simeq \mathrm{GL}\left(m, \varepsilon q^{e}\right)$ for some $m \geq 0$. Since $\langle\rho\rangle \unlhd R$, there exists a unique block $B_{\rho}$ of $K$ such that

$$
\left(1, b^{G}\right) \leq\left(\langle\rho\rangle, B_{\rho}\right) \leq(R, b) .
$$

Let $B_{\rho}=B_{\rho, 0} \times B_{\rho,+}$, where $B_{\rho, 0}, B_{\rho,+}$ are blocks of $K_{0}, K_{+}$respectively. Then $B_{\rho, 0} \subseteq \mathscr{E}_{r}\left(K_{0},\left(s_{0}\right)\right)$ and $B_{\rho,+} \subseteq \mathscr{E}_{r}\left(K_{+},\left(s_{+}\right)\right)$for some $s_{0} \in K_{0}^{*}$ and $s_{+} \in K_{+}^{*}$. By $(3 \mathrm{C}) s_{0} \times s_{+}$and $s$ are conjugate in $G^{*}$ and we may suppose $s=$ $s_{0} \times s_{+}$, so that this gives a decomposition of $s$. Moreover, the decomposition depends only on $b^{G}$ not on the choice of $R$. Indeed there exists a defect group $D$ of $b^{G}$ such that $Z(D) \leq Z(R) \leq R \leq D$, so that $V_{0}=C_{V}(D)$ and $V_{+}=[V, D]$ and a primary element of $D$ is a primary element of $R$. Thus we may suppose $\rho \in Z(D)$ is a primary element of $D$ and then the decomposition $s=s_{0} \times s_{+}$is determined by $b^{G}$. Suppose now $V=V_{+}$. Then $B_{\rho}=B_{\rho,+}$ and $B_{\rho} \subseteq \mathscr{E}_{r}(K,(s))$. Since $C_{G}(R)=C_{K}(R)$, we may view $(R, b)$ as a Brauer pair of $K$ and then $(R, b)$ has a Broue labeling $(R, t,-)$, where $t \in K^{*}$. Here, the third component of the label is empty since $K \simeq \mathrm{GL}\left(m, \varepsilon q^{e}\right)$ and $R$ acts fixed-point freely on the underlying space of $K$. By definition of normal inclusion of Brauer pairs, $\left(1, B_{\rho}\right) \leq(R, b)$ holds in $K$ and by [7, (3.2)], $t$ and $s$ are conjugate in $K^{*}$. In particular, $t$ determines a unique conjugacy class of $G^{*}$. We then give $(R, b)$ the label $(R, t,-)$.

Given $\Gamma$ in $\mathscr{F}$, let $e_{\Gamma}, \alpha_{\Gamma}$, and $m_{\Gamma}$ be the following integers: $e_{\Gamma}$ is the multiplicative order of $q^{2 \delta_{\Gamma}}$ or $\varepsilon_{\Gamma} q^{\delta_{\Gamma}}$ modulo $r$ according as $\Gamma \in \mathscr{F}_{0}$ or $\Gamma \epsilon$ $\mathscr{F}_{1} \cup \mathscr{F}_{2}, r^{\alpha_{\Gamma}}=\left(d_{\Gamma}\right)_{r}$, and $m_{\Gamma} e r^{\alpha_{\Gamma}}=\delta_{\Gamma} e_{\Gamma}$. In addition, let $\beta_{\Gamma}=1$ or 2 according as $\Gamma \in \mathscr{F}_{1} \cup \mathscr{F}_{2}$ or $\Gamma \in \mathscr{F}_{0}$.

Suppose $\operatorname{dim} V$ is even and $s$ is a semisimple element of $I_{0}\left(V^{*}\right)$ with primary decomposition

$$
V^{*}=\sum_{\Gamma} V_{\Gamma}^{*}(s), \quad s=\prod_{\Gamma} s(\Gamma) .
$$

We define a semisimple element $s^{*}$ of $I_{0}(V)$, which is determined uniquely up to conjugacy in $I(V)$, as follows: If $V$ is orthogonal, then $V$ and $V^{*}$ have the same dimension and type, so that $m_{\Gamma}(s)$ and $\eta_{\Gamma}(s)$ satisfy the relations (1.11). Thus a semisimple element, denoted by $s^{*}$, exists in $I(V)$ such that $m_{\Gamma}\left(s^{*}\right)=m_{\Gamma}(s)$ and $\eta_{\Gamma}\left(s^{*}\right)=\eta_{\Gamma}(s)$. Since $s \in G^{*}$, it follows that $s^{*} \in G$. If $V$ is symplectic, then $V^{*}$ is an odd dimensional orthogonal space. Let $\eta_{\Gamma}=1$ for all $\Gamma \in \mathscr{F}$, and $n_{\Gamma}=m_{\Gamma}(s)$ except when $\Gamma=X-1$, in which case, $n_{\Gamma}=m_{\Gamma}(s)-1$. Then $n_{\Gamma}$ and $\eta_{\Gamma}$ satisfy the relations (1.11) with $m_{\Gamma}(s)$ and $\eta_{\Gamma}(s)$ replaced by $n_{\Gamma}$ and $\eta_{\Gamma}$ respectively. So a semisimple element, denote by $s^{*}$, exists in $G$ such that $m_{\Gamma}\left(s^{*}\right)=n_{\Gamma}$ and $\eta_{\Gamma}\left(s^{*}\right)=\eta_{\Gamma}=1$. Thus $s^{*}$ is 
uniquely determined up to conjugacy in $I(V)$ and $\operatorname{det} s^{*}=1$. We shall call $s^{*}$ a dual of $s$.

The following proposition is due to Fong and Olsson.

(3D). The dual mapping $s \mapsto s^{*}$ induces a bijection $f:(s) \mapsto\left(s^{*}\right)$ from the conjugacy classes of r-elements of $I_{0}\left(V^{*}\right)$ onto the conjugacy classes of r-elements of $I_{0}(V)$ such that

$$
C_{I_{0}(V)}\left(s^{*}\right) \simeq C_{I_{0}\left(V^{*}\right)}(s)^{*}
$$

Proof. Suppose $s$ is an $r$-element and decomposes as (3.3). Then -1 is not an eigenvalue of $s$, so that $\operatorname{dim} V_{\Gamma}^{*}(s)=m_{\Gamma}(s) d_{\Gamma}$ and $\eta_{\Gamma}(s)=\varepsilon^{m_{\Gamma}(s)}$ for $\Gamma \neq X-1$. Thus

$$
m_{X-1}(s)=\operatorname{dim} V^{*}-\sum_{\Gamma \neq X-1} \operatorname{dim} V_{\Gamma}^{*}(s)
$$

and

$$
\eta_{X-1}(s)=(-1)^{(q-1) / 2 m_{X-1}(s) m_{X+1}(s)} \eta\left(V^{*}\right) \prod_{\Gamma \neq X-1} \eta_{\Gamma}(s),
$$

so that $s$ is determined uniquely up to conjugacy in $I\left(V^{*}\right)$ by its multiplicity function $m_{\Gamma}(s)$. Moreover, $s \in I_{0}\left(V^{*}\right)$ and the $I\left(V^{*}\right)$-class of $s$ decomposes into one or two conjugacy classes of $I_{0}\left(V^{*}\right)$ according as 1 is or is not an eigenvalue of $s$. Similar statements hold for $r$-elements of $I(V)$. If $V$ is symplectic, then the dual mapping induces a bijection of the conjugacy classes of $r$-elements of $I_{0}\left(V^{*}\right)$ onto the conjugacy classes of $r$-elements of $I_{0}(V)$. If $V$ and $V^{*}$ are even dimensional orthogonal spaces, then the dual mapping induces a bijection of the conjugacy classes of $r$-elements of $I\left(V^{*}\right)$ onto the conjugacy classes of $r$-elements of $I(V)$. Moreover, the $I\left(V^{*}\right)$-class of $s$ is a single $I_{0}\left(V^{*}\right)$-class if and only if the $I(V)$-class of $s^{*}$ is a single $I_{0}(V)$-class. So the dual mapping induces a bijection of the conjugacy classes of $r$-elements of $I_{0}\left(V^{*}\right)$ and $I_{0}(V)$. The isomorphism (3.4) follows by [12, (3A)].

Given $m \geq 1$, let $V$ be a symplectic or orthogonal space of dimension $2 \mathrm{em}$ and type $\varepsilon^{m}$ if $V$ is orthogonal. Let $G=I_{0}(V)$ and $G^{*}=I_{0}\left(V^{*}\right)$. By [12, (5.2)] $G$ has a basic subgroup $R$ of the form $R_{m, 0,0}$, and we denote by $u^{*}$ a primary element of $R$ and $u$ a dual of $u^{*}$ given by (3D), so that $\left|u^{*}\right|=r^{a}$, $u^{*}=u^{*}(\Gamma)$ for a unique $\Gamma \in \mathscr{F}_{1} \cup \mathscr{F}_{2}$, and $C_{G}\left(u^{*}\right)=C_{I(V)}\left(u^{*}\right) \simeq \mathrm{GL}\left(m, \varepsilon q^{e}\right)$. Moreover, the subgroup $\left\langle u^{*}\right\rangle$ is uniquely determined up to conjugacy in $I(V)$. Namely, if $v^{*} \in G$ is an element of order $r^{a}$ and $v^{*}=v^{*}\left(\Gamma^{\prime}\right)$ for a unique $\Gamma^{\prime} \in \mathscr{F}_{1} \cup \mathscr{F}_{2}$, then $\left\langle v^{*}\right\rangle$ and $\left\langle u^{*}\right\rangle$ are conjugate in $I(V)$. Let $\mathscr{S}$ and $\mathscr{S}^{*}$ be the sets of conjugacy classes of $G$ and $G^{*}$ of semisimple elements in the $r$-sections containing $u^{*}$ and $u$ respectively. Here the $r$-section containing $u^{*}$ in $G$, by definition, is the set of all elements in $G$ whose $r$-part is conjugate with $u^{*}$ in $G$. Thus each class of $\mathscr{S}$ has the form $\left(h^{*} u^{*}\right)$ for some semisimple $r^{\prime}$-element $h^{*} \in C_{G}\left(u^{*}\right)$. Define

$$
\mathscr{S}^{\prime}=\left\{\left[h^{*}\right]:\left(h^{*} u^{*}\right) \in \mathscr{S}\right\}, \quad \mathscr{S}^{* \prime}=\left\{[s]:(s u) \in \mathscr{S}^{*}\right\},
$$

where $\left[h^{*}\right]$ and $[s]$ are conjugacy classes of $h^{*}$ and $s$ in $I(V)$ and $I\left(V^{*}\right)$ respectively. 
(3E). The dual mapping $s \mapsto s^{*}$ from the semisimple elements of $I_{0}\left(V^{*}\right)$ to the semisimple elements of $I_{0}(V)$ induces a bijection $f:[s] \mapsto\left[s^{*}\right]$ from $\mathscr{S}^{* 1}$ onto $\mathscr{S}^{\prime}$ such that

$$
C_{I_{0}(V)}\left(u^{*}, s^{*}\right) \simeq C_{I_{0}\left(V^{*}\right)}(u, s) .
$$

Proof. Let $[s] \in \mathscr{S}^{* \prime}, s^{*}$ a dual of $s$ in $G, K=C_{G}\left(u^{*}\right)$, and $K^{*}=C_{G^{*}}(u)$, so that $K^{*}$ is a dual of $K$. Then $s$ and $s^{*}$ have primary decompositions

$$
V=\sum_{\Gamma} V_{\Gamma}\left(s^{*}\right), \quad s^{*}=\prod_{\Gamma} s^{*}(\Gamma), \quad V^{*}=\sum_{\Gamma} V_{\Gamma}^{*}(s), \quad s=\prod_{\Gamma} s(\Gamma) .
$$

Thus $C_{I\left(V^{*}\right)}(s)=\prod_{\Gamma} C_{\Gamma}(s)$, where $C_{\Gamma}(s)=C_{I\left(V_{\Gamma}^{*}(s)\right)}(s(\Gamma)$. Moreover, by $(1.10)$

$$
C_{\Gamma}(s) \simeq \begin{cases}I\left(V_{\Gamma}^{*}(s)\right) & \text { if } \Gamma \in \mathscr{F}_{0}, \\ \mathrm{GL}\left(m_{\Gamma}(s), \varepsilon_{\Gamma} q_{\Gamma}^{\delta_{\Gamma}}\right) & \text { if } \Gamma \in \mathscr{F}_{1} \cup \mathscr{F}_{2} .\end{cases}
$$

Let $u_{\Gamma}$ be the restriction of $u$ to $V_{\Gamma}^{*}(s)$. Then $\left[V_{\Gamma}^{*}(s), u_{\Gamma}\right]=V_{\Gamma}^{*}(s)$ for $\Gamma \neq$ $X-1$ and $u_{\Gamma} \in C_{\Gamma}(s)$. Thus

$$
m_{\Gamma}(s)= \begin{cases}e_{\Gamma} w_{\Gamma}(s) & \text { if } \Gamma \in \mathscr{F}_{1} \cup \mathscr{F}_{2}, \\ 2 e w_{\Gamma}(s) & \text { if } \Gamma \in \mathscr{F}_{0} \text { and } \operatorname{dim} V^{*} \text { is even, } \\ 2 e w_{\Gamma}(s) & \text { if } \Gamma=X+1 \text { and } \operatorname{dim} V^{*} \text { is odd } \\ 2 e w_{\Gamma}(s)+1 & \text { if } \Gamma=X-1 \text { and } \operatorname{dim} V^{*} \text { is odd, }\end{cases}
$$

for some integer $w_{\Gamma}(s)$, and $\eta_{X+1}(s)=\varepsilon^{w_{X+1}(s)}, \eta_{\Gamma}(s)=\varepsilon_{\Gamma}^{m_{\Gamma}(s)}$ for $\Gamma \in \mathscr{F}_{1} \cup \mathscr{F}_{2}$. Moreover, $\eta_{X-1}(s)$ is determined by the equation

$$
\eta\left(V^{*}\right)=(-1)^{(q-1) / 2 m_{X-1}(s) m_{X+1}(s)} \prod_{\Gamma} \eta_{\Gamma}(s) .
$$

Thus the type function $\eta_{\Gamma}(s)$ is uniquely determined by the multiplicity function $m_{\Gamma}(s)$, so that $[s]=\left[s^{\prime}\right]$ for $[s],\left[s^{\prime}\right] \in \mathscr{S}^{* \prime}$ if and only if $m_{\Gamma}(s)=$ $m_{\Gamma}\left(s^{\prime}\right)$ for all $\Gamma \in \mathscr{F}$. It is clear that $C_{K^{*}}(s)=C_{G^{*}}(u, s)=C_{C_{G^{*}}(s)}(u)$ and $C_{K^{*}}(s)=\prod_{\Gamma} C_{\Gamma}(u, s)$, where $C_{\Gamma}(u, s)=C_{C_{\Gamma}(s)}\left(u_{\Gamma}\right)$ for $\Gamma \in \mathscr{F}_{1} \cup \mathscr{F}_{2}$ and $C_{\Gamma}(u, s)=C_{I_{0}\left(V_{\Gamma}^{*}(s)\right)}\left(u_{\Gamma}\right)$ for $\Gamma \in \mathscr{F}$. By (3.7) and (3.8)

$$
C_{\Gamma}(u, s) \simeq \mathrm{GL}\left(w_{\Gamma}(s), \varepsilon_{\Gamma} q^{e_{\Gamma} \delta_{\Gamma}}\right)
$$

for all $\Gamma \in \mathscr{F}$. Similarly, $C_{I(V)}\left(s^{*}\right)=\prod_{\Gamma} C_{\Gamma}\left(s^{*}\right)$, where $C_{\Gamma}\left(s^{*}\right)=C_{I\left(V_{\Gamma}\left(s^{*}\right)\right)}\left(s^{*}(\Gamma)\right)$. Moreover,

$$
C_{\Gamma}\left(s^{*}\right)= \begin{cases}I\left(V_{\Gamma}\left(s^{*}\right)\right) & \text { if } \Gamma \in \mathscr{F}_{0}, \\ \operatorname{GL}\left(m_{\Gamma}\left(s^{*}\right), \varepsilon_{\Gamma} q^{\delta_{\Gamma}}\right) & \text { if } \Gamma \in \mathscr{F}_{1} \cup \mathscr{F}_{2} .\end{cases}
$$

By definition of $s^{*}, m_{\Gamma}\left(s^{*}\right)=m_{\Gamma}(s)$ except when $\Gamma=X-1$ and $V$ is symplectic, in which case, $m_{\Gamma}\left(s^{*}\right)=m_{\Gamma}(s)-1$. Thus $m_{\Gamma}\left(s^{*}\right)=\beta_{\Gamma} e_{\Gamma} w_{\Gamma}(s)$, where $\beta_{\Gamma}=1$ or 2 according as $\Gamma \in \mathscr{F}_{1} \cup \mathscr{F}_{2}$ or $\Gamma \in \mathscr{F}_{0}$. Let $w_{\Gamma}(s)=\sum_{\beta} n_{\beta} r^{\beta}$ be the $r$-adic expansion of $w_{\Gamma}(s)$, and $\mathbf{c}_{\beta}=(1,1, \ldots, 1)$ ( $\beta$ terms). Then a Sylow $r$-subgroup $D(\Gamma)$ of $C_{\Gamma}\left(s^{*}\right)$ is of the form $\prod_{\beta}\left(R_{m_{\Gamma}, \alpha_{\Gamma}, 0, \varepsilon_{\beta}}\right)^{n_{\beta}}$. Thus a Sylow $r$-subgroup $P$ of $C_{I(V)}\left(s^{*}\right)$ is of the form $\prod_{\Gamma} D(\Gamma)$ as a subgroup of $I(V)$, so that $P$ has a primary element $v^{*}$ and $\left\langle v^{*}\right\rangle$ is conjugate with $\left\langle u^{*}\right\rangle$ in $I(V)$. Thus a conjugate of $s^{*}$ in $I(V)$ lies in $K$. Replacing $s^{*}$ by its conjugate, we may suppose $s^{*} \in K$. So $C_{K}\left(s^{*}\right)=C_{G}\left(u^{*}, s^{*}\right)=C_{C_{G}\left(s^{*}\right)}\left(u^{*}\right)$ and 
if $u_{\Gamma}^{*}$ is the restriction of $u^{*}$ to $V_{\Gamma}\left(s^{*}\right)$, then $C_{K}\left(s^{*}\right)=\prod_{\Gamma} C_{\Gamma}\left(u^{*}, s^{*}\right)$, where $C_{\Gamma}\left(u^{*}, s^{*}\right)=C_{C_{\Gamma}\left(s^{*}\right)}\left(u_{\Gamma}^{*}\right)$. Moreover,

$$
C_{\Gamma}\left(u^{*}, s^{*}\right) \simeq \mathrm{GL}\left(w_{\Gamma}(s), \varepsilon_{\Gamma} q^{e_{\Gamma} \delta_{\Gamma}}\right),
$$

for all $\Gamma \in \mathscr{F}$. Since $s^{*}$ is an $r^{\prime}$-element and $s^{*} \in K$, it follows $\left(s^{*} u^{*}\right) \in \mathscr{S}$ and $\left[s^{*}\right] \in \mathscr{S}^{\prime}$.

Conversely, given $\left[s^{*}\right] \in \mathscr{S}^{\prime}$, suppose $s^{*}$ decomposes as (3.6). Since $u^{*} \in$ $C_{G}\left(s^{*}\right)$ and the restriction $u_{\Gamma}^{*}$ of $u^{*}$ to $V_{\Gamma}\left(s^{*}\right)$ lies in $C_{\Gamma}\left(s^{*}\right)$, it follows $m_{\Gamma}\left(s^{*}\right)=\beta_{\Gamma} e_{\Gamma} w_{\Gamma}\left(s^{*}\right)$. Define $n_{\Gamma}=m_{\Gamma}\left(s^{*}\right)$ except when $\Gamma=X-1$ and $V$ is symplectic, in which case, $n_{\Gamma}=m_{\Gamma}\left(s^{*}\right)+1$. In addition, define $\eta_{\Gamma}=\varepsilon_{\Gamma}^{m_{\Gamma}\left(s^{*}\right)}$ for $\Gamma \in \mathscr{F}_{1} \cup \mathscr{F}_{2}, \eta_{X+1}=\varepsilon^{w_{X+1}\left(s^{*}\right)}$, and $\eta_{X-1}$ is chosen so that (3.9) holds with $\eta_{\Gamma}(s)$ and $m_{\Gamma}(s)$ replaced by $\eta_{\Gamma}$ and $n_{\Gamma}$ respectively. Thus $n_{\Gamma}$ and $\eta_{\Gamma}$ satisfy the relation (1.11) for $V^{*}$ with $m_{\Gamma}(s)$ and $\eta_{\Gamma}(s)$ replaced by $n_{\Gamma}$ and $\eta_{\Gamma}$, so that a semisimple element, denote by $s$, exists in $I_{0}\left(V^{*}\right)$ such that $m_{\Gamma}(s)=n_{\Gamma}$ and $\eta_{\Gamma}(s)=\eta_{\Gamma}$. Such an element is determined uniquely up to conjugacy in $I\left(V^{*}\right)$. Thus $m_{\Gamma}(s)$ satisfy equation (3.8) with $w_{\Gamma}(s)$ replaced by $w_{\Gamma}\left(s^{*}\right)$. A similar proof to above shows that a Sylow $r$-subgroup of $C_{I\left(V^{*}\right)}(s)$ has a primary element conjugate with $u$ in $I\left(V^{*}\right)$. We may suppose $u \in C_{I\left(V^{*}\right)}(s)$ and $(s u) \in \mathscr{S}^{*}$, so that $[s] \in \mathscr{S}^{* \prime}$. But $[s]=\left[s^{\prime}\right]$ for $[s],\left[s^{\prime}\right] \in \mathscr{S}^{*}$ if and only if $m_{\Gamma}(s)=m_{\Gamma}\left(s^{\prime}\right)$ for all $\Gamma \in \mathscr{F}$, so the two maps induced by $s \mapsto s^{*}$ and $s^{*} \mapsto s$ are inverse each other and both are bijections. The isomorphism (3.5) follows by (3.10) and (3.11).

Remark. As shown in the proof of $(3 \mathrm{E})$, if $s^{*}$ is a semisimple $r^{\prime}$-element of $I_{0}(V)$ such that a Sylow $r$-subgroup of $C_{I(V)}\left(s^{*}\right)$ acts fixed-point freely on $V$, then $m_{\Gamma}\left(s^{*}\right)=\beta_{\Gamma} e_{\Gamma} w_{\Gamma}\left(s^{*}\right)$, so that a dual $s$ of $s^{*}$ is a well-defined semisimple $r^{\prime}$-element of $I_{0}\left(V^{*}\right)$. Moreover, if $u^{*}$ is a primary element of a Sylow $r$ subgroup of $C_{I(V)}\left(s^{*}\right)$ and $u$ is its dual, then we may suppose $u$ is a primary element of a Sylow $r$-subgroup of $C_{I\left(V^{*}\right)}(s)$ and $C_{I_{0}(V)}\left(u^{*}, s^{*}\right) \simeq C_{I_{0}\left(V^{*}\right)}(u, s)$.

(3F). Given integer $m \geq 1$, let $V$ be a symplectic or orthogonal space over $\mathbb{F}_{q}$ of dimension $2 e m$ and $\eta(V)=\varepsilon^{m}$ if $V$ is orthogonal. Let $G=I_{0}(V)$, and $B$ a block of $G$ contained in $\mathscr{E}_{r}(G,(s))$ for some semisimple $r^{\prime}$-element $s$ of $G^{*}$. If a defect group $R$ of $B$ acts fixed-point freely on $V$, then $R$ is conjugate in $I(V)$ with a Sylow r-subgroup of $C_{G}\left(s^{*}\right)$, where $s^{*}$ is a dual of $s$ in $G$.

Proof. Since $R$ is radical in $I(V)$, it has a primary element $z^{*}$. Let $K=$ $C_{G}\left(z^{*}\right)$ and $K^{*}$ its dual. Then $z^{*}=z^{*}(\Gamma)$ for a unique $\Gamma \in \mathscr{F}_{1} \cup \mathscr{F}_{2}, K=$ $C_{I(V)}\left(z^{*}\right) \simeq \mathrm{GL}\left(m, \varepsilon q^{e}\right)$, and $K^{*}$ is embedded as a regular subgroup in $G^{*}$. Suppose $\left(z^{*}, B_{z^{*}}\right)$ is a major subsection associated with $B$, in the sense of [6], and $B_{z^{*}} \subseteq \mathscr{E}_{r}(K,(t))$. Then $s$ and $t$ are conjugate in $G^{*}$ by (3C) and $R$ is a defect group of $B_{z^{*}}$. Replace $s$ by a conjugate we may suppose $s=t$, so that $R$ is conjugate with a Sylow $r$-subgroup of $C_{K^{*}}(s)^{*}$ in $K$ by a result of $[11, \S 5]$. Let $s^{*}$ be a dual of $s$ and $\rho$ an element of order $r^{a}$ in $Z\left(K^{*}\right)$. Such an element $\rho$ exists since $K \simeq K^{*}$. Thus $K^{*} \leq C_{G^{*}}(\rho)$ and $\delta_{\Gamma}=e$ for all $\Gamma \in \mathscr{F}_{1} \cup \mathscr{F}_{2}$ with $m_{\Gamma}(\rho) \neq 0$. By (1.9) and (1.10) $C_{G^{*}}(\rho)=K^{*}$, so that $\rho$ is a primary element of $O_{r}\left(Z\left(K^{*}\right)\right)$. Thus $\langle\rho\rangle$ is conjugate in $I\left(V^{*}\right)$ with the subgroup generated by a dual of $z^{*}$ given by (3D). Replacing $\rho$ by $\rho^{k}$ for some integer $k$, we may suppose $\rho$ is a dual of $z^{*}$. Since $s$ lies in the $r$-section containing $\rho$, we may suppose $s^{*}$ lies in the $r$-section containing $z^{*}$ 
and $C_{K}\left(s^{*}\right) \simeq C_{K^{*}}(s)$ by $(3 \mathrm{E})$. By (3.10) and (3.11) $C_{K}\left(s^{*}\right)$ and $C_{K^{*}}(s)^{*}$ are conjugate in $K$. Thus $R$ is conjugate with a Sylow $r$-subgroup of $C_{K}\left(s^{*}\right)$.

We may suppose $R$ is a Sylow $r$-subgroup of $C_{K}\left(s^{*}\right)$. Let $P$ be a Sylow $r$ subgroup of $C_{I(V)}\left(s^{*}\right)$ containing $R$ and $u^{*}$ a primary element of $P$. So $u^{*} \in$ $Z(P), R \leq C_{P}\left(z^{*}\right) \leq C_{K}\left(s^{*}\right)$, and $R=C_{P}\left(z^{*}\right)$ since $R$ is Sylow in $C_{K}\left(s^{*}\right)$. Thus $u^{*} \in Z(R)$ and $u^{*}$ is a primary element of $R$. So $\left\langle z^{*}\right\rangle=\left\langle u^{*}\right\rangle \leq Z(P)$, $P=C_{P}\left(z^{*}\right)=R$, and $(3 \mathrm{~F})$ holds.

Let $\mathscr{F}^{\prime}$ be the subsets of polynomials in $\mathscr{F}$ whose roots have $r^{\prime}$-order. Given $\Gamma \in \mathscr{F}^{\prime}$, we shall define $G_{\Gamma}, R_{\Gamma}, C_{\Gamma}, \theta_{\Gamma}$, and $s_{\Gamma}$ as follows: Let $V_{\Gamma}$ denote a symplectic or orthogonal space of dimension $2 e_{\Gamma} \delta_{\Gamma}$ over $\mathbb{F}_{q}$ and of type $\varepsilon_{\Gamma}^{e_{\Gamma}}$ or $\varepsilon$ according as $\Gamma \in \mathscr{F}_{1} \cup \mathscr{F}_{2}$ or $\Gamma \in \mathscr{F}_{0}$ if $V_{\Gamma}$ is orthogonal. Thus $I\left(V_{\Gamma}\right)$ has a primary element $s_{\Gamma}^{*}$ with a unique elementary divisor $\Gamma$ of multiplicity of $\beta_{\Gamma} e_{\Gamma}$ and $I\left(V_{\Gamma}\right)$ has a basic subgroup $R_{\Gamma}$ of form $R_{m_{\Gamma}, \alpha_{\Gamma}, 0}$ by $[12,(1.12)$ and $(5.2)]$. Let $G_{\Gamma}=I\left(V_{\Gamma}\right), G_{\Gamma}^{0}=I_{0}\left(V_{\Gamma}\right)$, and $C_{\Gamma}=C_{G_{\Gamma}}\left(R_{\Gamma}\right)$. Then $s_{\Gamma}^{*} \in G_{\Gamma}^{0}$ and $C_{\Gamma} \simeq \mathrm{GL}\left(m_{\Gamma}, \varepsilon q^{e^{a_{\Gamma}}}\right)$, so that a Coxeter torus $T_{\Gamma}$ of $C_{\Gamma}$ has order $q^{m_{\Gamma} e^{a_{\Gamma}}}-\varepsilon^{m_{\Gamma}}$. The dual $T_{\Gamma}^{*}$ is embedded as a regular subgroup of $C_{\Gamma}^{*}$, and in turn, $C_{\Gamma}^{*}$ is embedded as a regular subgroup of $G_{\Gamma}^{0 *}$. We claim that there exists an element $s_{\Gamma}$ in $T_{\Gamma}^{*}$ such that $C_{C_{\Gamma}^{*}}\left(s_{\Gamma}\right)=T_{\Gamma}^{*}$ and as an element of $G_{\Gamma}^{0 *}, s_{\Gamma}$ and $s_{\Gamma}^{*}$ are dual each other in the sense of $(3 \mathrm{E})$. Indeed

$$
C_{G_{\Gamma}}\left(s_{\Gamma}^{*}\right)= \begin{cases}I\left(V_{\Gamma}\right) & \text { if } \Gamma=X \pm 1, \\ G L\left(e_{\Gamma}, \varepsilon_{\Gamma} q^{\delta_{\Gamma}}\right) & \text { if } \Gamma \neq X \pm 1,\end{cases}
$$

so that a Sylow $r$-subgroup of $C_{G_{\Gamma}}\left(s_{\Gamma}^{*}\right)$ acts fixed-point freely on $V_{\Gamma}$. By the remark of $(3 \mathrm{E})$ a dual $s_{\Gamma}$ of $s_{\Gamma}^{*}$ exists in $G_{\Gamma}^{0 *}$ and

$$
C_{G_{\Gamma}^{0 *}}\left(s_{\Gamma}\right)= \begin{cases}\mathrm{GL}\left(e_{\Gamma}, \varepsilon_{\Gamma} q^{\delta_{\Gamma}}\right) & \text { if } \Gamma \neq X \pm 1, \\ \operatorname{SO}^{\varepsilon}(2 e, q) & \text { if } \Gamma=X \pm 1 \text { and } V_{\Gamma} \text { is orthogonal, } \\ \left\langle w, 1 \times \mathrm{SO}^{\varepsilon}(2 e, q)\right\rangle & \text { if } \Gamma=X+1 \text { and } V_{\Gamma} \text { is symplectic, } \\ \mathrm{SO}(2 e+1, q) & \text { if } \Gamma=X-1 \text { and } V_{\Gamma} \text { is symplectic, }\end{cases}
$$

where $w$ is an element in $\operatorname{SO}\left(V_{\Gamma}^{*}\right)$ such that $w^{2} \in 1 \times \operatorname{SO}^{\varepsilon}(2 e, q)$, and 1 is the identity matrix of size 1 . Let $R_{\Gamma}^{\prime *}$ be a Sylow $r$-subgroup of $C_{G_{\Gamma}^{0 *}}\left(s_{\Gamma}\right), C_{\Gamma}^{\prime *}=$ $C_{G_{\Gamma}^{0 *}}\left(R_{\Gamma}^{\prime *}\right)$, and $T_{\Gamma}^{\prime *}=C_{C_{\Gamma}^{\prime *}}\left(s_{\Gamma}\right)$. Then $s_{\Gamma} \in T_{\Gamma}^{\prime *}$ and $T_{\Gamma}^{\prime *}=C_{C_{G_{\Gamma}^{0 *}}\left(s_{\Gamma}\right)}\left(R_{\Gamma}^{\prime *}\right)$. Thus $T_{\Gamma}^{\prime *}$ has order $q^{e_{\Gamma} \delta_{\Gamma}}-\varepsilon_{\Gamma}^{e_{\Gamma}}$. But $e_{\Gamma} \delta_{\Gamma}=m_{\Gamma} e r^{\alpha_{\Gamma}}, r$ divides both $q^{m_{\Gamma} e^{a_{\Gamma}}}-\varepsilon^{m_{\Gamma}}$ and $q^{e_{\Gamma} \delta_{\Gamma}}-\varepsilon_{\Gamma}^{e_{\Gamma}}$, so $\varepsilon_{\Gamma}^{e_{\Gamma}}=\varepsilon^{m_{\Gamma}}$ and $R_{\Gamma}^{\prime *}$ is a Sylow $r$-subgroup of $G_{\Gamma}^{0 *}$. In particular, $R_{\Gamma}^{\prime *}$ is cyclic of order $r^{a+\alpha_{\Gamma}}$ and has type $R_{m_{\Gamma}, \alpha_{\Gamma}, 0}$ as a subgroup of $I\left(V_{\Gamma}^{*}\right)$. Let $R_{\Gamma}^{*}$ be the Sylow $r$-subgroup of $T_{\Gamma}^{*}$. Then $R_{\Gamma}^{*}$ is cyclic of order $r^{a+\alpha_{\Gamma}}$ and there exists $g \in I\left(V_{\Gamma}^{*}\right)$ such that $\left(R_{\Gamma}^{*}\right)^{g}=R_{\Gamma}^{\prime *}$, so that $\left(C_{\Gamma}^{*}\right)^{g}=C_{\Gamma}^{\prime *}$. Thus $\left(T_{\Gamma}^{*}\right)^{g h}=T_{\Gamma}^{\prime *}$, and $s_{\Gamma}^{h^{-1}} g^{-1} \in T_{\Gamma}^{*}$ for some $h \in C_{\Gamma}^{\prime *}$. Thus $s_{\Gamma}^{h^{-1}} g^{-1}$ is a dual of $s_{\Gamma}^{*}$ in $G_{\Gamma}^{0 *}$ and $C_{C_{\Gamma}^{*}}\left(s_{\Gamma}^{h^{-1} g^{-1}}\right)=T_{\Gamma}^{*}$. We may denote $s_{\Gamma}^{h^{-1} g^{-1}}$ by $s_{\Gamma}$ and then the claim holds. By $(3 \mathrm{E}) s_{\Gamma}$ is uniquely determined by $\Gamma$ up to conjugacy in $I\left(V_{\Gamma}^{*}\right)$.

Let $\phi_{\Gamma}$ be the character of $T_{\Gamma}$ corresponding to $s_{\Gamma}$, and let

$$
\theta_{\Gamma}= \pm R_{T_{\Gamma}}^{C_{\Gamma}}\left(\phi_{\Gamma}\right)=\grave{ \pm} R_{T_{\Gamma}^{*}}^{C_{\Gamma}^{*}}\left(s_{\Gamma}\right)
$$

where the sign is chosen so that $\theta_{\Gamma}$ is an irreducible character of $C_{\Gamma}$. The 
block $b_{\Gamma}$ of $C_{\Gamma}$ containing $\theta_{\Gamma}$ then has defect group $R_{\Gamma}$ by $[11,(4 \mathrm{C})]$ and the Brauer pair $\left(R_{\Gamma}, b_{\Gamma}\right)$ of $G_{\Gamma}^{0}$ has the label $\left(R_{\Gamma}, s_{\Gamma},-\right)$.

(3G). Let $N_{\Gamma}=N_{G_{\Gamma}}\left(R_{\Gamma}\right)$, and $N\left(\theta_{\Gamma}\right)$ the stabilizer of $\theta_{\Gamma}$ in $N_{\Gamma}$.

(a) $\left(N\left(\theta_{\Gamma}\right): C_{\Gamma}\right)=\beta_{\Gamma} e_{\Gamma}$. In particular, $\left|\operatorname{Irr}^{0}\left(N\left(\theta_{\Gamma}\right), \theta_{\Gamma}\right)\right|=\beta_{\Gamma} e_{\Gamma}$ and $R_{\Gamma}$ is a defect group of $b_{\Gamma}^{G_{\Gamma}}$.

(b) Let $\Gamma, \Gamma^{\prime} \in \mathscr{F}^{\prime}$ such that $G_{\Gamma}=G_{\Gamma^{\prime}}$ and $R_{\Gamma}=R_{\Gamma^{\prime}}$, so that $C_{\Gamma}=C_{\Gamma^{\prime}}$ and $N_{\Gamma}=N_{\Gamma^{\prime}}$. Let $\theta_{\Gamma}$ and $\theta_{\Gamma^{\prime}}$ be the canonical characters of $b_{\Gamma}$ and $b_{\Gamma^{\prime}}$ respectively. Then $b_{\Gamma}^{\tau}=b_{\Gamma^{\prime}}$ for some $\tau \in N_{\Gamma}$ if and only if $s_{\Gamma}$ and $s_{\Gamma^{\prime}}$ are conjugate in $I\left(V_{\Gamma}^{*}\right)$, where $V_{\Gamma}^{*}$ is the underlying space of $G_{\Gamma}^{0 *}$.

Proof. (a) It suffices to show $\left(N\left(\theta_{\Gamma}\right): C_{\Gamma}\right)=\beta_{\Gamma} e_{\Gamma}$ since $N_{\Gamma} / C_{\Gamma}$ is cyclic of order $2 e r^{\alpha_{\Gamma}}$. If $\Gamma \in \mathscr{F}_{0}$, then $C_{\Gamma}=T_{\Gamma}, \theta_{\Gamma}=\phi_{\Gamma}$, and $\theta_{\Gamma}$ is either the identity character or the character of order 2 of $T_{\Gamma}$. Thus $N\left(\theta_{\Gamma}\right)=N_{\Gamma}$ and $\left(N\left(\theta_{\Gamma}\right): C_{\Gamma}\right)=2 e_{\Gamma}$.

Suppose $\Gamma \in \mathscr{F}_{1} \cup \mathscr{F}_{2}$, so that $T_{\Gamma}=C_{C_{\Gamma}}(\rho)$ for some $\rho \in T_{\Gamma}$ and $T_{\Gamma}=$ $C_{G_{\Gamma}}(\mu \rho)$ for any generator $\mu$ of $R_{\Gamma}$. Let $\Delta$ be the unique elementary divisor of $\mu \rho$ and $N\left(T_{\Gamma}\right)=N_{G_{\Gamma}}\left(T_{\Gamma}\right)$. Following [12, p. 149], if $\Delta \in \mathscr{T}_{1}$, we have $N\left(T_{\Gamma}\right)=\left\langle\sigma, T_{\Gamma}\right\rangle$, where $\sigma: t \mapsto t^{q}$ for $t \in T_{\Gamma}$. Here $\sigma$ has order $2 m_{\Gamma} e r^{\alpha_{\Gamma}}$ in $N\left(T_{\Gamma}\right) / T_{\Gamma}$ and $\sigma^{m_{\Gamma} e r_{\Gamma}{ }_{\Gamma}}$ inverts $T_{\Gamma}$. If $\Delta \in \mathscr{F}_{2}$, we have $N\left(T_{\Gamma}\right)=\left\langle\beta, \gamma, T_{\Gamma}\right\rangle$, where $\beta: t \mapsto t^{q}, \gamma: t \mapsto t^{-1}$ for $t \in T_{\Gamma}$. Here $\beta$ and $\gamma$ have order $m_{\Gamma} e r^{\alpha_{\Gamma}}$ and 2 respectively in $N\left(T_{\Gamma}\right) / T_{\Gamma}$. Moreover, $N_{\Gamma}=N\left(T_{\Gamma}\right) C_{\Gamma}$.

Let $N_{\Gamma}$ act on the pairs $(T, \phi)$ by conjugation and let $[T, \phi]$ be the $C_{\Gamma^{-}}$ orbit of the pair $(T, \phi)$, where $T$ is a Coxeter torus of $C_{\Gamma}$ and $\phi$ is an irreducible character of $T$. Then $N_{\Gamma}$ induces an action on the $C_{\Gamma}$-orbits and the $N_{\Gamma}$-orbit of $\left[T_{\Gamma}, \phi_{\Gamma}\right]$ consists of $\left\{\left[T_{\Gamma}, \phi_{\Gamma}^{ \pm q^{l}}\right]\right\}$, where $1 \leq l \leq m_{\Gamma} e r^{\alpha_{\Gamma}}$. Moreover, we claim that for $\tau \in N_{\Gamma}, \varrho \in N\left(T_{\Gamma}\right),\left[T_{\Gamma}, \phi_{\Gamma}\right]^{\tau}=\left[T_{\Gamma}, \phi_{\Gamma}^{\varrho}\right]$ if and only if $\left(R_{T_{\Gamma}}^{C_{\Gamma}}\left(\phi_{\Gamma}\right)\right)^{\tau}=R_{T_{\Gamma}}^{C_{\Gamma}}\left(\phi_{\Gamma}^{\varrho}\right)$. Indeed given $\tau \in N_{\Gamma}$, then $T_{\Gamma}^{\tau \varpi}=T_{\Gamma}$ for some $\varpi \epsilon$ $C_{\Gamma}$ and $\left(R_{T_{\Gamma}}^{C_{\Gamma}}\left(\phi_{\Gamma}\right)\right)^{\tau}=\left(R_{T_{\Gamma}}^{C_{\Gamma}}\left(\phi_{\Gamma}\right)\right)^{\tau \varpi}=R_{T_{\Gamma}}^{C_{\Gamma}}\left(\phi_{\Gamma}^{\tau \varpi}\right)$. Thus $\left[T_{\Gamma}, \phi_{\Gamma}\right]^{\tau}=\left[T_{\Gamma}, \phi_{\Gamma}^{\varrho}\right]$ if and only if $\left[T_{\Gamma}, \phi_{\Gamma}^{\tau \varpi}\right]=\left[T_{\Gamma}, \phi_{\Gamma}^{\varrho}\right]$ if and only if $\left(R_{T_{\Gamma}}^{C_{\Gamma}}\left(\phi_{\Gamma}\right)\right)^{\tau}=R_{T_{\Gamma}}^{C_{\Gamma}}\left(\phi_{\Gamma}^{\varrho}\right)$. Thus the claim holds. In particular, $N\left(\theta_{\Gamma}\right)$ is the stabilizer of $\left[T_{\Gamma}, \phi_{\Gamma}\right]$ in $N_{\Gamma}$.

The group $C_{\Gamma}^{*}$ acts on the pairs $\left(T^{*}, s\right)$ of Coxeter torus $T^{*}$ and $s \in T^{*}$ by conjugation. Let $\left[T^{*}, s\right]$ be the conjugacy $C_{\Gamma}^{*}$-class of $\left(T^{*}, s\right)$. By $[18,(7.5)]$ the $C_{\Gamma}$-classes $[T, \phi]$ are in bijection with the $C_{\Gamma}^{*}$-classes $\left[T^{*}, s\right]$ and if $[T, \phi]$ corresponds to $\left[T^{*}, s\right]$, then $R_{T}^{C_{\Gamma}}(\phi)=R_{T^{*}}^{C_{\Gamma}^{*}}(s)$ and $\left[T, \phi^{k}\right]$ corresponds to $\left[T^{*}, s^{k}\right]$ for any integer $k$. Let $R_{\Gamma}^{*}$ be the Sylow $r$-subgroup of $T_{\Gamma}^{*}$ and $N_{\Gamma}^{*}=$ $N_{I\left(V_{\Gamma}^{*}\right)}\left(R_{\Gamma}^{*}\right)$. Then $\left|R_{\Gamma}^{*}\right|=r^{a+\alpha_{\Gamma}}, R_{\Gamma}^{*} \leq Z\left(C_{\Gamma}^{*}\right)$, and $R_{\Gamma}^{*}$ has form $R_{m_{\Gamma}, \alpha_{\Gamma}, 0}$ as a subgroup of $I\left(V_{\Gamma}^{*}\right)$. So $C_{\Gamma}^{*}=C_{I_{0}\left(V_{\Gamma}^{*}\right)}\left(R_{\Gamma}^{*}\right)$. Let $N\left(T_{\Gamma}^{*}\right)=N_{I\left(V_{\Gamma}^{*}\right)}\left(T_{\Gamma}^{*}\right)$. Then $N_{\Gamma}^{*}=N\left(T_{\Gamma}^{*}\right) C_{\Gamma}^{*}$ and $N_{\Gamma}^{*}$ acts on the pairs $\left(T^{*}, s\right)$ by conjugation, so that $N_{\Gamma}^{*}$ induces an action on classes $\left[T^{*}, s\right]$. If $G_{\Gamma}$ is a symplectic group, then $I\left(V_{\Gamma}^{*}\right) \simeq \mathrm{O}\left(\beta_{\Gamma} e_{\Gamma} d_{\Gamma}+1, q\right)$, and the action of $N\left(T_{\Gamma}^{*}\right)$ on $T_{\Gamma}^{*}$ is similar to that of $N\left(T_{\Gamma}\right)$ on $T_{\Gamma}$, namely for $g \in N\left(T_{\Gamma}^{*}\right), g$ acts on $T_{\Gamma}^{*}$ by $g: t \mapsto t^{ \pm q^{l}}$, where $t \in T_{\Gamma}^{*}$ and $1 \leq l \leq m_{\Gamma} e r^{\alpha_{\Gamma}}$. If $G_{\Gamma}$ is an orthogonal group, then $I\left(V_{\Gamma}^{*}\right) \simeq$ $I\left(V_{\Gamma}\right) \simeq \mathrm{O}^{ \pm}\left(\beta_{\Gamma} e_{\Gamma} d_{\Gamma}, q\right)$ and the action of $N\left(T_{\Gamma}^{*}\right)$ on $T_{\Gamma}^{*}$ is similar to that of $N\left(T_{\Gamma}\right)$ on $T_{\Gamma}$. Thus the $N_{\Gamma}^{*}$-orbit of $\left[T_{\Gamma}^{*}, s_{\Gamma}\right]$ consists of $\left\{\left[T_{\Gamma}^{*}, s_{\Gamma}^{ \pm q^{l}}\right]\right\}$, where $1 \leq l \leq m_{\Gamma} e^{\alpha_{\Gamma}}$ and the elements in this orbit are in bijection with that in the $N_{\Gamma}$-orbit of $\left[T_{\Gamma}, \phi_{\Gamma}\right]$. So $\left(N_{\Gamma}: N\left(\theta_{\Gamma}\right)\right)=\left(N_{\Gamma}^{*}: N\left(\left[T_{\Gamma}^{*}, s_{\Gamma}\right]\right)\right)$, where $N\left(\left[T_{\Gamma}^{*}, s_{\Gamma}\right]\right)$ 
is the stabilizer of $\left[T_{\Gamma}^{*}, s_{\Gamma}\right]$ in $N_{\Gamma}^{*}$. Let $H^{*}=N\left(\left[T_{\Gamma}^{*}, s_{\Gamma}\right]\right)$ or $N\left(\left[T_{\Gamma}^{*}, s_{\Gamma}\right]\right) \cap$ $I_{0}\left(V_{\Gamma}^{*}\right)$ according as $V_{\Gamma}$ is orthogonal or symplectic. Then $H^{*} \geq C_{\Gamma}^{*}$ and $\left|N\left(\theta_{\Gamma}\right)\right|=\left|H^{*}\right|$ since $\left|N_{\Gamma}\right|=\left|N_{\Gamma}^{*}\right|$ or $\frac{1}{2}\left|N_{\Gamma}^{*}\right|$ according as $V_{\Gamma}$ is orthogonal or symplectic. Moreover, $\left(N\left(\theta_{\Gamma}\right): C_{\Gamma}\right)=\left(H^{*}: C_{\Gamma}^{*}\right)$.

Now fix the $C_{\Gamma}^{*}$-classes $\left[T_{\Gamma}^{*}, s_{\Gamma}\right]$. Then it is clear that $C_{\Gamma}^{*}$ and $H^{*}$ act transitively on the class and so $\left(H^{*}: N_{H^{*}}\left(T_{\Gamma}^{*}, s_{\Gamma}\right)\right)=\left(C_{\Gamma}^{*}: N_{C_{\Gamma}^{*}}\left(T_{\Gamma}^{*}, s_{\Gamma}\right)\right)$, where $N_{H^{*}}\left(T_{\Gamma}^{*}, s_{\Gamma}\right)$ and $N_{C_{\Gamma}^{*}}\left(T_{\Gamma}^{*}, s_{\Gamma}\right)$ are the stabilizers of the pair $\left(T_{\Gamma}^{*}, s_{\Gamma}\right)$ in $H^{*}$ and $C_{\Gamma}^{*}$ respectively. But $H^{*} \geq C_{\Gamma}^{*}, N_{C_{\Gamma}^{*}}\left(T_{\Gamma}^{*}, s_{\Gamma}\right)=T_{\Gamma}^{*}$, and

$$
\left(H^{*}: T_{\Gamma}^{*}\right)=\left(H^{*}: C_{\Gamma}^{*}\right)\left(C_{\Gamma}^{*}: T_{\Gamma}^{*}\right)=\left(H^{*}: N_{H^{*}}\left(T_{\Gamma}^{*}, s_{\Gamma}\right)\right)\left(N_{H^{*}}\left(T_{\Gamma}^{*}, s_{\Gamma}\right): T_{\Gamma}^{*}\right),
$$

so $\left(H^{*}: C_{\Gamma}^{*}\right)=\left(N_{H^{*}}\left(T_{\Gamma}^{*}, s_{\Gamma}\right): T_{\Gamma}^{*}\right)$. If $V_{\Gamma}$ is orthogonal, then $C_{I\left(V_{\Gamma}^{*}\right)}\left(s_{\Gamma}\right)=$ $C_{I_{0}\left(V_{\Gamma}^{*}\right)}\left(s_{\Gamma}\right)$ by $\Gamma \in \mathscr{F}_{1} \cup \mathscr{F}_{2}$. Thus in any case $N_{H^{*}}\left(T_{\Gamma}^{*}, s_{\Gamma}\right) \leq I_{0}\left(V_{\Gamma}^{*}\right)$. Let $K^{*}=$ $C_{I_{0}\left(V_{\Gamma}^{*}\right)}\left(s_{\Gamma}\right)$. Then $K^{*} \simeq \mathrm{GL}\left(e_{\Gamma}, \varepsilon_{\Gamma} q^{\delta_{\Gamma}}\right)$ and $N_{H^{*}}\left(T_{\Gamma}^{*}, s_{\Gamma}\right)=N_{K^{*}}\left(T_{\Gamma}^{*}\right)$. Since $T_{\Gamma}^{*}$ is a Coxeter torus of $K^{*},\left(N_{K^{*}}\left(T_{\Gamma}^{*}\right): T_{\Gamma}^{*}\right)=e_{\Gamma}$ and then $\left(N\left(\theta_{\Gamma}\right): C_{\Gamma}\right)=e_{\Gamma}$.

(b) Let $\theta_{\Gamma^{\prime}}= \pm R_{\Gamma_{\Gamma^{\prime}}^{*}}^{C_{\Gamma^{\prime}}^{*}}\left(s_{\Gamma^{\prime}}\right)$. Suppose $\theta_{\Gamma}^{\tau}=\theta_{\Gamma^{\prime}}$ for some $\tau \in N_{\Gamma}$. Then $\left[T_{\Gamma}, \phi_{\Gamma}\right]^{\tau}$ corresponds to $\left[T_{\Gamma}^{*}, s_{\Gamma}^{n}\right]$ for some $n \in N\left(T_{\Gamma}^{*}\right)$ since the elements in the $N_{\Gamma}$-orbit of $\left[T_{\Gamma}, \phi_{\Gamma}\right]$ are in bijection with elements in the $N_{\Gamma}^{*}$-orbit of $\left[T_{\Gamma}^{*}, s_{\Gamma}\right]$ and $N_{\Gamma}^{*}=N\left(T_{\Gamma}^{*}\right) C_{\Gamma}^{*}$. Thus $\theta_{\Gamma}^{\tau}= \pm R_{T_{\Gamma}^{*}}^{C_{\Gamma}^{*}}\left(s_{\Gamma}^{n}\right)$ and $\left[T_{\Gamma}^{*}, s_{\Gamma}^{n}\right]=\left[T_{\Gamma^{\prime}}^{*}, s_{\Gamma^{\prime}}\right]$. So $s_{\Gamma}$ is conjugate with $s_{\Gamma^{\prime}}$ in $I\left(V^{*}\right)$. Conversely, suppose $s_{\Gamma}$ and $s_{\Gamma^{\prime}}$ are conjugate in $I\left(V_{\Gamma}^{*}\right)$. Since $T_{\Gamma}^{*}$ and $T_{\Gamma^{\prime}}^{*}$ are Coxeter tori of $C_{\Gamma}^{*}, T_{\Gamma^{\prime}}^{* c}=T_{\Gamma}^{*}$ and $s_{\Gamma^{\prime}}^{c}=s_{\Gamma}^{w}$ for some $c \in C_{\Gamma}^{*}$ and $w \in I\left(V_{\Gamma}^{*}\right)$. If $\Gamma \in \mathscr{F}_{0}$, then $C_{\Gamma}^{*}=T_{\Gamma}^{*}=T_{\Gamma^{\prime}}^{*}$ and $s_{\Gamma^{\prime}}=s_{\Gamma}^{w}$, so that both $s_{\Gamma^{\prime}}$ and $s_{\Gamma}$ are elements of $T_{\Gamma}^{*}$ of order 1 or 2 according as $\Gamma=X-1$ or $\Gamma=X+1$. Thus $s_{\Gamma^{\prime}}=s_{\Gamma}$ and $\theta_{\Gamma^{\prime}}=\theta_{\Gamma}$. Suppose $\Gamma \in \mathscr{F}_{1} \cup \mathscr{F}_{2}$, so that $K^{*}=C_{I_{0}\left(V_{\Gamma}^{*}\right)}\left(s_{\Gamma^{\prime}}\right)^{c w^{-1}}$ and hence $T_{\Gamma}^{*}, T_{\Gamma^{\prime}}^{* c w^{-1}}$ are Coxeter tori of $K^{*}$. So $T_{\Gamma}^{* g}=T_{\Gamma^{\prime}}^{* c w^{-1}}$ for some $g \in K^{*}, T_{\Gamma}^{* g w}=T_{\Gamma^{\prime}}^{* c}=T_{\Gamma}^{*}$, and $g w \in N_{\Gamma}^{*}$. It follows that

$$
\left[T_{\Gamma}^{*}, s_{\Gamma}\right]^{g w}=\left[T_{\Gamma}^{*}, s_{\Gamma}^{w}\right]=\left[T_{\Gamma}^{* c^{-1}}, s_{\Gamma}^{w c^{-1}}\right]=\left[T_{\Gamma^{\prime}}^{*}, s_{\Gamma^{\prime}}\right] .
$$

Since $g w \in N_{\Gamma}^{*},\left[T_{\Gamma}^{*}, s_{\Gamma}\right]^{g w}$ corresponds to $\left[T_{\Gamma}, \phi_{\Gamma}\right]^{\tau}$ for some $\tau \in N_{\Gamma}$ and then $\theta_{\Gamma^{\prime}}=\theta_{\Gamma}^{\tau}$. This completes the proof.

Remark. Let $G_{\Gamma}$ be an orthogonal group, and $N_{0}\left(\theta_{\Gamma}\right)=N\left(\theta_{\Gamma}\right) \cap G_{\Gamma}^{0}$. By [12, (6B)] $\left(N\left(\theta_{\Gamma}\right): N_{0}\left(\theta_{\Gamma}\right)\right)=\beta_{\Gamma}$.

For each $\alpha \geq 0$ and $m \geq 0$, let $V_{m, \alpha, 0}$ denote a symplectic or orthogonal space over $\mathbb{F}_{q}$ of dimension $2 m e r^{\alpha}$ and type $\varepsilon^{m}$ if $V_{m, \alpha, 0}$ is orthogonal. Thus $I\left(V_{m, \alpha, 0}\right)$ has a basic subgroup of form $R_{m, \alpha, 0}$ (see $\left.\S 2\right)$.

(3H). Let $G=I\left(V_{m, \alpha, 0}\right), R=R_{m, \alpha, 0}$ a basic subgroup of $G, b$ a block of $C_{G}(R) R$ with defect group $R$, and $\theta$ the canonical character of $b$. If $N(\theta)$ is the stabilizer of $\theta$ in $N_{G}(R)$ and $\left(N(\theta): C_{G}(R) R\right)_{r}=1$, then $G=G_{\Gamma}$, $R=R_{\Gamma}$, and $\theta=\theta_{\Gamma}$ for some $\Gamma \in \mathscr{F}^{\prime}$.

Proof. Let $C=C_{G}(R), N=N_{G}(R)$, and $G_{0}=I_{0}\left(V_{m, \alpha, 0}\right)$. Then $C=C_{G_{0}}(R)$ and $N / C$ is cyclic of order $2 e r^{\alpha}$.

Since $C \simeq \mathrm{GL}\left(m, \varepsilon q^{e r^{\alpha}}\right)$, it follows by $[11,(4 \mathrm{~B})$ and $(4 \mathrm{C})]$ that

$$
\theta=\varepsilon_{T} R_{T}^{C}(\phi)
$$

where $\varepsilon_{T}= \pm 1, T$ is a Coxeter torus of $C$ and $\phi$ is an $r$-rational irreducible character of $T$. Moreover, the dual $T^{*}$ is embedded as a regular subgroup of 
$C^{*}$, and $C^{*}$ is embedded as a regular subgroup of $G_{0}^{*}$. There is an element $s$ of $T^{*}$ such that $s$ corresponds to $\phi$ and $T^{*}=C_{C^{*}}(s)$. In particular, if $\phi^{2}=1$, then $s^{2}=1, T^{*}=C^{*}, m=1$, and $\theta=\phi$. Thus $N=N(\theta)$ and $(N(\theta): C)_{r}=(N: C)_{r}=1$, so that $\alpha=0$. In this case $R=R_{X \pm 1}$, and $\theta=\theta_{X \pm 1} \quad$ (see $[12$, p. 148]).

Suppose $\phi^{2} \neq 1$. Then as an element of $C^{*}, s$ has a unique elementary divisor $\Delta$ with multiplicity 1 since $T^{*}=C_{C^{*}}(s)$ is the Coxeter torus of $C^{*}$. Regard $s$ as an element of $G_{0}^{*}$. By [12, (9A) and (9.2)] there is a unique $\Gamma \in \mathscr{F}_{1} \cup \mathscr{F}_{2}$ such that the multiplicity of $\Gamma$ in $s$ is $e_{\Gamma} r^{l}$ and $e_{\Gamma} r^{l} d_{\Gamma}=2 m e r^{\alpha}$ for some $l \geq 0$. So $C_{G_{0}^{*}}(s) \simeq \mathrm{GL}\left(e_{\Gamma} r^{l}, \varepsilon_{\Gamma} q^{\delta_{\Gamma}}\right)$. A similar proof to that of (3G)(a) shows that $(N(\theta): C)=\left(N_{C_{G_{0}^{*}(s)}}\left(T^{*}\right): T^{*}\right)=e_{\Gamma} r^{l}$. Thus $l=0$ and $e_{\Gamma} d_{\Gamma}=2 m e r^{\alpha}$ since $(N(\theta): C)_{r}=1$. But $(m, r)=1$ by [11, (4B)]. It follows that $m=m_{\Gamma}, \alpha=\alpha_{\Gamma}$, and $G=G_{\Gamma}, R=R_{\Gamma}, \theta=\theta_{\Gamma}$. This completes the proof.

Given $\Gamma \in \mathscr{F}^{\prime}$ and $\gamma \geq 0$. Let

$$
V_{\Gamma, \gamma}=V_{\Gamma} \perp V_{\Gamma} \perp \cdots \perp V_{\Gamma},
$$

where there are $r^{\gamma}$ terms $V_{\Gamma}$ on the right-hand side. Then if $V_{\Gamma}$ is orthogonal, $V_{\Gamma, \gamma}$ has type $\left(\varepsilon_{\Gamma}\right)^{e_{\Gamma} r^{\gamma}}=\varepsilon_{\Gamma}^{e_{\Gamma}}$ or $\varepsilon^{\gamma^{\gamma}}=\varepsilon$ according as $\Gamma \in \mathscr{F}_{1} \cup \mathscr{F}_{2}$ or $\Gamma \in \mathscr{F}_{0}$.

(3I). Let $G=I\left(V_{\Gamma, \gamma}\right), R=R_{m_{\Gamma}, \alpha_{\Gamma}, \gamma}$ a basic subgroup of $G$, and $C=C_{G}(R)$. Then $C=C_{\Gamma} \otimes I_{\gamma}$, where $I_{\gamma}$ is the identity matrix of order $r^{\gamma}$. The irreducible character $\theta=\theta_{\Gamma} \otimes I_{\gamma}$, of $C$ defined by $\theta\left(c \otimes I_{\gamma}\right)=\theta_{\Gamma}(c)$ for $c \in C_{\Gamma}$ is then a character of defect 0 of $C R / R$, and $\left|\operatorname{Irr}^{0}(N(\theta), \theta)\right|=\beta_{\Gamma} e_{\Gamma}$.

Proof. The proof is essentially the same as that of $(3 \mathrm{~A})$, except that the automorphisms on $C=C_{\Gamma} \otimes I_{\gamma}$ induced by $N(R)$ have order $2 e r^{\alpha_{\Gamma}}$, and their actions are the same as the automorphisms on $C_{\Gamma}$ induced by $N_{\Gamma} / C_{\Gamma}$.

Remark. Suppose $G=I\left(V_{\Gamma, \gamma}\right)$ is an orthogonal group. Let $G_{0}=I_{0}\left(V_{\Gamma, \gamma}\right)$ and $N_{0}(\theta)=N(\theta) \cap G_{0}$. Then $\left|N(\theta): N_{0}(\theta)\right|=\beta_{\Gamma}$ and for each $\psi \in \operatorname{Irr}^{0}(N(\theta), \theta)$ the restriction $\left.\psi\right|_{N_{0}(\theta)}$ of $\psi$ to $N_{0}(\theta)$ is irreducible. Indeed let $N^{0}=$ $\{g \in N:[g, Z(R)]=1\}$. Then $N^{0} \leq N_{0}(\theta)$ and in the notation of (3A), $N(\theta)=N(\vartheta)$ and $N(\vartheta) / N^{0} \simeq N\left(\theta_{\Gamma}\right) / C_{\Gamma}$, where $\vartheta$ is the unique irreducible character of $N^{0}$ covering $\theta$ and having defect 0 as a character of $N^{0} / R$. The remark of $(3 \mathrm{G})$ implies $\left|N(\theta): N_{0}(\theta)\right|=\beta_{\Gamma}$. Since $\psi$ covers $\vartheta$ and $N(\vartheta) / N^{0}$ is cyclic, $\left.\psi\right|_{N^{0}}=\vartheta$ is irreducible, so that $\left.\psi\right|_{N_{0}(\theta)}$ is irreducible. This completes the proof.

Given $\Gamma \in \mathscr{F}^{\prime}$, and $d \geq 0$. Let $G=I\left(V_{\Gamma, d}\right)$, and $R=R_{m_{\Gamma}, \alpha_{\Gamma}, \gamma, \mathrm{c}}$ be a basic subgroup of $G$, where $\mathbf{c}=\left(c_{1}, c_{2}, \ldots, c_{l}\right)$, and $\gamma+c_{1}+c_{2}+\cdots+c_{l}=d$. Then $C=C_{G}(R)=C_{\Gamma} \otimes I_{\gamma} \otimes I_{\mathrm{c}}$, where $I_{\gamma}$ and $I_{\mathrm{c}}$ are the identity matrices of order $r^{\gamma}$ and $r^{c_{1}+c_{2}+\cdots+c_{l}}$ respectively. The irreducible character of $C$ defined by

$$
\theta\left(c \otimes I_{\gamma} \otimes I_{\mathbf{c}}\right)=\theta_{\Gamma}(c)
$$

for $c \in C_{\Gamma}$ is a character of defect 0 of $C R / R$. We shall say that the pair $(R, \theta)$ is of type $\Gamma$. If $(R, \theta)$ is of type $\Gamma$, then $\theta$ is a canonical character of a block $b$ of $C$ with defect group $Z(R)$, and the Brauer pair $(R, b)$ of $G$ is also a Brauer pair of $G_{0}=I_{0}\left(V_{\Gamma, d}\right)$ since $C=C_{G_{0}}(R)$. Let $D$ be 
the base subgroup of $R=R_{m_{\Gamma}, \alpha_{\Gamma}, \gamma}\left\{A_{\mathbf{c}}\right.$. Then each component $Q$ of $D$ is of the form $R_{m_{\Gamma}, \alpha_{\Gamma}, \gamma}$, so that by the remark of (1C) $Q$ contains a normal subgroup $Q^{\prime}$ such that $C_{I_{0}\left(V_{m_{\Gamma}, a_{\Gamma}, \gamma}\right)}\left(Q^{\prime}\right)=C_{I\left(V_{\left.m_{\Gamma}, a_{\Gamma}, \gamma\right)}\right.}\left(Q^{\prime}\right)=\prod_{i=1}^{r^{\gamma}} C_{i}$ is a regular subgroup of $I_{0}\left(V_{m_{\Gamma}, \alpha_{\Gamma}, \gamma}\right)$, where $V_{m_{\Gamma}, \alpha_{\Gamma}, \gamma}$ is the underlying space of $Q$ and $C_{i} \simeq \mathrm{GL}\left(m_{\Gamma}, \varepsilon q^{e r^{\alpha} \Gamma}\right)$ for all $i$. Let $R^{\prime}$ be the subgroup of $D$ with each component $Q$ of $D$ replaced by $Q^{\prime}$. Then $R^{\prime}$ is a normal subgroup of $R$ and $C^{\prime}=C_{G}\left(R^{\prime}\right)=\prod_{i=1}^{r^{d}} C_{i}$, where $C_{i} \simeq \mathrm{GL}\left(m_{\Gamma}, \varepsilon q^{e r^{a \Gamma}}\right)$ for all $1 \leq i \leq r^{d}$. Thus $C^{\prime}$ is a regular subgroup of $I_{0}\left(V_{\Gamma, d}\right)$ and $C \leq C^{\prime}$, so that $C^{\prime *}$ is embedded as a regular subgroup of $I_{0}\left(V_{\Gamma, d}\right)^{*}$. Now we may suppose $C_{i}=C_{\Gamma}$ and $s_{\Gamma} \in C_{i}^{*}$ for all $i$. Let

$$
x_{\Gamma}=s_{\Gamma} \times s_{\Gamma} \times \cdots \times s_{\Gamma} \quad\left(r^{d} \text { times }\right)
$$

be an element of $C^{\prime *}$ and $x_{\Gamma}^{*}$ a dual of $x_{\Gamma}$ in $G$. Then as an element of $G, x_{\Gamma}^{*}$ has a unique elementary divisor $\Gamma$ of multiplicity $\beta_{\Gamma} e_{\Gamma} r^{d}$ and type $\eta_{\Gamma}\left(x_{\Gamma}^{*}\right)=\eta\left(V_{\Gamma, d}\right)$. The subgroup $C_{\Gamma}^{*} \otimes I_{\gamma} \otimes I_{\mathrm{c}}$ can be regarded as a diagonal subgroup of $C^{\prime *}$, so that $s_{\Gamma} \otimes I_{\gamma} \otimes I_{\mathrm{c}} \in C^{\prime *}$ and $x_{\Gamma}$ is conjugate with $s_{\Gamma} \otimes I_{\gamma} \otimes I_{\mathrm{c}}$ in $I\left(V^{*}\right)$. Thus $(R, b)$ is labeled by $\left(R, x_{\Gamma},-\right)$. The Brauer pair $(R, b)$ of $G$ will also be denoted by $(R, \theta)$.

(3J). (a) Let $G=I(V), R$ a basic subgroup of $G,(R, \varphi)$ a weight of $G$, and $\theta$ an irreducible character of $C_{G}(R)$ covered by $\varphi$. Then $(R, \theta)$ is of type $\Gamma$ for some $\Gamma \in \mathscr{F}^{\prime}$.

(b) The pair $(R, \theta)$ of $G$ with type $\Gamma$ is uniquely determined by $\Gamma$ up to conjugacy in $N=N_{G}(R)$, that is, if $\left(R, \theta^{\prime}\right)$ is another pair with type $\Gamma$, then $\theta^{\prime}=\theta^{n}$ for some $n \in N$.

Proof. (a) Suppose $V=V_{m, \alpha, \gamma, \mathbf{c}}$ and $R=R_{m, \alpha, \gamma, \mathbf{c}}$, where $\mathbf{c}=\left(c_{1}, \ldots, c_{l}\right)$. Let $G_{1}=I\left(V_{m, \alpha, 0}\right), R_{1}=R_{m, \alpha, 0}$ a basic subgroup of $G_{1}, C_{1}=C_{G_{1}}\left(R_{1}\right)$, and $N_{1}=N_{G_{1}}\left(R_{1}\right)$. Then $C_{1} \simeq \mathrm{GL}\left(m, \varepsilon q^{e r^{\alpha}}\right)$ and $C=C_{G}(R)=C_{1} \otimes I_{\gamma} \otimes I_{\mathrm{c}}$. Thus $\theta$ has the form $\theta_{1} \otimes I_{y} \otimes I_{\mathrm{c}}$, where $\theta_{1}$ is a character of $C_{1}$. Since $\theta$ has defect 0 as a character of $C / Z(R), \theta_{1}$ has defect 0 as a character on $C_{1} / R_{1}$. The block of $C_{1}$ containing $\theta_{1}$ has defect group $R_{1}$.

Let $R_{m, \alpha, \gamma}$ a basic subgroup of $I\left(V_{m, \alpha, \gamma}\right), N_{m, \alpha, \gamma}$ and $C_{m, \alpha, \gamma}$ the normalizer and centralizer of $R_{m, \alpha, \gamma}$ in $I\left(V_{m, \alpha, \gamma}\right)$. Then $C_{m, \alpha, \gamma}=C_{1} \otimes I_{\gamma}$ and $\left(\theta_{1} \otimes I_{\gamma}\right)\left(c \otimes I_{\gamma}\right)=\theta_{1}(c)$ for $c \in C_{1}$ is an irreducible character of $C_{m, \alpha, \gamma}$. By $(2.5)$

$$
\begin{aligned}
N & =\left(N_{m, \alpha, \gamma} / R_{m, \alpha, \gamma}\right) \otimes N_{\mathbf{S}(u)}\left(A_{\mathbf{c}}\right), \\
N / R & \simeq\left(N_{m, \alpha, \gamma} / R_{m, \alpha, \gamma}\right) \times \operatorname{GL}\left(c_{1}, r\right) \times \cdots \times \mathrm{GL}\left(c_{l}, r\right),
\end{aligned}
$$

where $u=r^{c_{1}+\cdots+c_{l}}$. If $N_{m, \alpha, \gamma}^{0}=\left\{g \in N_{m, \alpha, \gamma}:\left[g, Z\left(R_{m, \alpha, \gamma}\right)\right]=1\right\}$, then $N_{m, \alpha, \gamma} / N_{m, \alpha, \gamma}^{0} \simeq N_{1} / C_{1}$. Let $\varphi=I(\psi)$ for some $\psi \in \operatorname{Irr}^{0}(N(\theta) ; \theta)$, and $N\left(\theta_{1} \otimes I_{\gamma}\right)$ be the stabilizer of $\theta_{1} \otimes I_{\gamma}$ in $N_{m, \alpha, \gamma}$. Then

$$
N(\theta) / R \simeq\left(N\left(\theta_{1} \otimes I_{\gamma}\right) / R_{m, \alpha, \gamma}\right) \times \mathrm{GL}\left(c_{1}, r\right) \times \cdots \times \mathrm{GL}\left(c_{l}, r\right) .
$$

But $\psi$ is a character of defect 0 of $N(\theta) / R$, so it covers an irreducible character $\psi_{0}$ in $\operatorname{Irr}^{0}\left(N\left(\theta_{1} \otimes I_{\gamma}\right), \theta_{1} \otimes I_{\gamma}\right)$. Same proof as that of $(3 \mathrm{~A})$ shows that $N_{m, \alpha, \gamma}^{0} \leq$ $N\left(\theta_{1} \otimes I_{\gamma}\right)$ and $N_{m, \alpha, \gamma}^{0}$ has a unique irreducible character $\vartheta$ covering $\theta_{1} \otimes I_{\gamma}$ and having defect 0 as a character of $N_{m, \alpha, \gamma}^{0} / R_{m, \alpha, \gamma}$. Moreover, $N\left(\theta_{1} \otimes I_{\gamma}\right)=$ 
$N(\vartheta)$ and $N(\vartheta) / N_{m, \alpha, \gamma}^{0} \simeq N\left(\theta_{1}\right) / C_{1}$, where $N\left(\theta_{1}\right)$ is the stabilizer of $\theta_{1}$ in $N_{1}$. Thus $\psi_{0} \in \operatorname{Irr}^{0}(N(\vartheta), \vartheta)$ and $\psi_{0}(1)=\vartheta(1)$ since $N_{m, \alpha, \gamma} / N_{m, \alpha, \gamma}^{0}$ is cyclic. By $(3.1)\left(N(\vartheta): N_{m, \alpha, \gamma}^{0}\right)_{r}=1$ and hence $\left(N\left(\theta_{1}\right): C_{1}\right)_{r}=1$. It follows by $(3 \mathrm{H})$ that $G_{1}=G_{\Gamma}, R_{1}=R_{\Gamma}$, and $\theta_{1}=\theta_{\Gamma}$ for some $\Gamma \in \mathscr{F} \prime$. Thus $\left(R_{1}, \theta_{1}\right)$ is labeled by $\left(R_{1}, s_{\Gamma},-\right)$ and $(R, \theta)$ has type $\Gamma$, so (a) holds.

(b) Let $G=I\left(V_{\Gamma, d}\right), R=R_{m_{\Gamma}, \alpha_{\Gamma}, \gamma, \mathrm{c}}$ a basic subgroup of $G, C=C_{G}(R)$, $N=N_{G}(R), \theta=\theta_{\Gamma} \otimes I_{\gamma} \otimes I_{\mathrm{c}}$, and $\theta^{\prime}=\theta_{\Gamma}^{\prime} \otimes I_{\gamma} \otimes I_{\mathrm{c}}$, where $\theta_{\Gamma}, \theta_{\Gamma}^{\prime}$ are irreducible characters of $C_{\Gamma}$, and $\theta, \theta^{\prime}$ are defined as (3.13). If $\left(R_{\Gamma}, t_{\Gamma},-\right)$ and $\left(R_{\Gamma}, t_{\Gamma}^{\prime},-\right)$ are the labels of $\left(R_{\Gamma}, \theta_{\Gamma}\right)$ and $\left(R_{\Gamma}, \theta_{\Gamma}^{\prime}\right)$ respectively, then $t_{\Gamma}, t_{\Gamma}^{\prime}$ are conjugate in $G_{\Gamma}$ since both Brauer pairs $(R, \theta)$ and $\left(R, \theta^{\prime}\right)$ are labeled by $\left(R, x_{\Gamma},-\right)$. It follows by $(3 \mathrm{G})(\mathrm{b})$ that $\theta_{\Gamma}^{w}=\theta_{\Gamma}^{\prime}$ for some $w \in N_{\Gamma}$.

Let $C_{m_{\Gamma}, \alpha_{\Gamma}, \gamma}=C_{I\left(V_{\Gamma, \gamma}\right)}\left(R_{m_{\Gamma}, \alpha_{\Gamma}, \gamma}\right)$, so that $C_{m_{\Gamma}, \alpha_{\Gamma}, \gamma}=C_{\Gamma} \otimes I_{\gamma}$. Let $\theta_{\Gamma} \otimes$ $I_{\gamma}$ and $\theta_{\Gamma}^{\prime} \otimes I_{\gamma}$ be irreducible characters of $C_{m_{\Gamma}, \alpha_{\Gamma}, \gamma}$ defined as (3I). Since $N_{m_{\Gamma}, \alpha_{\Gamma}, \gamma} / N_{m_{\Gamma}, \alpha_{\Gamma}, \gamma}^{0} \simeq N_{\Gamma} / C_{\Gamma}$, it follows $\left(\theta \otimes I_{\gamma}\right)^{h}=\theta_{\Gamma}^{\prime} \otimes I_{\gamma}$ for some $h \in$ $N_{m_{\Gamma}, \alpha_{\Gamma}, \gamma}$ and so $\theta^{n}=\theta^{\prime}$ for some $n \in N$, where the structure of $N$ is given above with $m$ and $\alpha$ replaced by $m_{\Gamma}$ and $\alpha_{\Gamma}$ respectively.

Remark. Suppose $R$ is a basic subgroup of $G=I(V), b$ a block of $C_{G}(R) R$ with defect group $R$, and $\theta$ the canonical character of $b$. If $\left(N(\theta): C_{G}(R) R\right)_{r}$ $=1$, then $(R, \theta)$ is of type $\Gamma$ for some $\Gamma \in \mathscr{F}^{\prime}$. In particular, this occurs when $b$ is a root block of a block $B$ and $R$ is a defect group of $B$. Here a root block $b$ of a block $B$, in the sense of Brauer, is a block of $C_{G}(R) R$ with defect group $R$ such that $b^{G}=B$, where $R$ is a defect group of $B$. Thus if $b$ is a root block of $B$ and $\theta$ is the canonical character of $b$, then $(R, b)$ is a maximal Brauer pair containing $(1, B)$ and $\left(N(\theta): C_{G}(R) R\right)_{r}=1$, where $b$ is regarded as a block of $C_{G}(R)$. The proof of the remark is similar to that of $(3 \mathrm{~J})(\mathrm{a})$. Indeed in the notation of $(3 \mathrm{~J})(\mathrm{a}) N\left(\theta_{1} \otimes I_{\gamma}\right) / N_{m, \alpha, \gamma}^{0} \simeq N\left(\theta_{1}\right) / C_{1}$ and

$$
N(\theta) / C_{G}(R) R \simeq\left(N\left(\theta_{1} \otimes I_{\gamma}\right) / C_{m, \alpha, \gamma} R_{m, \alpha, \gamma}\right) \times \mathrm{GL}\left(c_{1}, r\right) \times \cdots \times \mathrm{GL}\left(c_{l}, r\right) .
$$

Thus $\left(N\left(\theta_{1} \otimes I_{\gamma}\right): C_{m, \alpha, \gamma} R_{m, \alpha, \gamma}\right)_{r}=1$ and $\left(N\left(\theta_{1} \otimes I_{\gamma}\right): N_{m, \alpha, \gamma}^{0}\right)_{r}=1$ since $(N(\theta): C(R) R)_{r}=1$. So $\left(N\left(\theta_{1}\right): C_{1}\right)_{r}=1$ and the block of $C_{1}$ containing $\theta_{1}$ has defect group $R_{1}$. By $(3 \mathrm{H}) G_{1}=G_{\Gamma}, R_{1}=R_{\Gamma}, \theta_{1}=\theta_{\Gamma}$, and $(R, \theta)$ has type $\Gamma$.

Following the remark above we can get a corollary.

(3K). Let $V$ be a symplectic or even dimensional orthogonal space, $G=I(V)$, $G_{0}=I_{0}(V)$, and let $B$ and $B^{\prime}$ be blocks of $G$ with defect $D$ and $D^{\prime}$ respectively such that $[V, D]=V=\left[V, D^{\prime}\right]$. Let $b$ and $b^{\prime}$ be root blocks of $B$ and $B^{\prime}$ respectively, $b^{G_{0}} \subseteq \mathscr{E}_{r}\left(G_{0},(s)\right)$, and $b^{\prime G_{0}} \subseteq \mathscr{E}_{r}\left(G_{0},\left(s^{\prime}\right)\right)$, where $s$ and $s^{\prime}$ are semisimple $r^{\prime}$-elements of $G_{0}^{*}$. Then $B=B^{\prime}$ if and only if $s$ and $s^{\prime}$ are conjugate in $I\left(V^{*}\right)$, where $V^{*}$ is the underlying space of $G_{0}^{*}$.

Proof. Since $D$ is radical in $G$, a primary element of $D$ exists and then $G$ has an $r$-subgroup of form $R_{m, 0,0}$ for some $m \geq 1$. By [12, (1.12) and (5.2)], $V$ has dimension 2em and type $\varepsilon^{m}$ if $V$ is orthogonal.

Suppose $s$ and $s^{\prime}$ are conjugate in $I\left(V^{*}\right)$, so that $s^{*}$ and $s^{\prime *}$ are conjugate in $G$ by definition. By (3F) $D$ and $D^{\prime}$ are conjugate with Sylow $r$-subgroups of $C_{G}\left(s^{*}\right)$ and $C_{G}\left(s^{\prime *}\right)$ respectively, so that they are conjugate in $G$. We may suppose $D=D^{\prime}$. 
By (2D) $V$ and $D$ have a corresponding decomposition,

$$
V=V_{1} \perp V_{2} \perp \cdots \perp V_{t}, \quad D=D_{1} \times D_{2} \times \cdots \times D_{t},
$$

where $D_{i}$ is a basic subgroup of $I\left(V_{i}\right)$. Let $\theta$ and $\theta^{\prime}$ be the canonical characters of $b$ and $b^{\prime}$ respectively. Thus $C=C_{G}(D)=\prod_{i} C_{i}, \theta=\prod_{i} \theta_{i}$, and $\theta^{\prime}=\prod_{i} \theta_{i}^{\prime}$, where $\theta, \theta^{\prime}$ are regarded as characters of $C$, and $\theta_{i}, \theta_{i}^{\prime}$ are characters of $C_{i}=C_{I\left(V_{i}\right)}\left(D_{i}\right)=C_{I_{0}\left(V_{i}\right)}\left(D_{i}\right)$. Since $b$ and $b^{\prime}$ are root blocks, it follows $(N(\theta): C D)_{r}=\left(N\left(\theta^{\prime}\right): C D\right)_{r}=1$. Let $N\left(\theta_{i}\right)$ and $N\left(\theta_{i}^{\prime}\right)$ be the stabilizers of $\theta_{i}$ and $\theta_{i}^{\prime}$ in $N_{I\left(V_{i}\right)}\left(D_{i}\right)$ respectively. Then $\left(N\left(\theta_{i}\right): C_{i} D_{i}\right)_{r}=$ $\left(N\left(\theta_{i}^{\prime}\right): C_{i} D_{i}\right)_{r}=1$ for all $i$. By the remark above, $\left(D_{i}, \theta_{i}\right)$ and $\left(D_{i}, \theta_{i}^{\prime}\right)$ are of type $\Gamma$ and $\Gamma^{\prime}$ respectively, where $\Gamma, \Gamma^{\prime} \in \mathscr{F}^{\prime}$.

Suppose $\left(D_{i}, t_{i},-\right)$ and $\left(D_{i}, t_{i}^{\prime},-\right)$ are labels of Brauer pairs $\left(D_{i}, \theta_{i}\right)$ and $\left(D_{i}, \theta_{i}^{\prime}\right)$ of $I\left(V_{i}\right)$ respectively. Let $z \in Z(D)$ be primary. Then we may suppose $t_{i}$ and $t_{i}^{\prime}$ are elements of $K_{i}^{*}$, where $K_{i}=C_{I\left(V_{i}\right)}\left(z_{i}\right)$ and $z_{i}$ is the restriction of $z$ to $V_{i}$. So $\left(D, \prod_{i} t_{i},-\right)$ is the label of $(D, b)$ and $\left(D, \prod_{i} t_{i}^{\prime},-\right)$ is the label of $\left(D, b^{\prime}\right)$. By $(3 C), s$ and $\prod_{i} t_{i}$ are conjugate in $G_{0}^{*}$, so are $s^{\prime}$ and $\prod_{i} t_{i}^{\prime}$. Thus these three elements $s, \prod_{i} t_{i}$, and $\prod_{i} t_{i}^{\prime}$ are conjugate in $I\left(V^{*}\right)$. Let $D(\Gamma)=\prod_{i} D_{i}, s(\Gamma)=\prod_{i} t_{i}, V(\Gamma)=\sum_{i} V_{i}, D^{\prime}\left(\Gamma^{\prime}\right)=\prod_{j} D_{j}$, and $s^{\prime}\left(\Gamma^{\prime}\right)=$ $\prod_{j} t_{j}^{\prime}$, where $i$ and $j$ runs over indices such that $\left(D_{i}, \theta_{i}\right)$ and $\left(D_{j}, \theta_{j}^{\prime}\right)$ have type $\Gamma$ and $\Gamma^{\prime}$ respectively. Then $\Pi_{\Gamma} s(\Gamma), \Pi_{\Gamma^{\prime}} s\left(\Gamma^{\prime}\right)$, and $s$ are conjugate in $I\left(V^{*}\right)$. Let $z_{\Gamma}$ be the restriction of $z$ to $V(\Gamma)$ and $K_{\Gamma}=C_{I(V(\Gamma))}\left(z_{\Gamma}\right)$. Then $K_{\Gamma}^{*}$ can be embedded as a subgroup of $I_{0}(V(\Gamma))^{*}$ and $s(\Gamma) \in K_{\Gamma}^{*}$. If $s(\Gamma)^{*}$ is a dual of $s(\Gamma)$ in $I_{0}(V(\Gamma))$, then $\prod_{\Gamma} s(\Gamma)^{*}$ is a primary decomposition of $s^{*}$. Similarly, $\prod_{\Gamma} s^{\prime}(\Gamma)^{*}$ is a primary decomposition of $s^{*}$. So $D(\Gamma)$ is a Sylow $r$-subgroup of $H_{\Gamma}=C_{I(V(\Gamma))}\left(s(\Gamma)^{*}\right)$, and $D(\Gamma), D^{\prime}(\Gamma)$ are conjugate in $G$.

If $\Gamma=X \pm 1$, then $\operatorname{dim} V(\Gamma)=2 e w_{\Gamma}$ for some $w_{\Gamma} \geq 0$ and $D(\Gamma)=$ $\prod_{\beta}\left(R_{1,0,0, \mathrm{c}_{\beta}}\right)^{n_{\beta}}$, where $n_{\beta} \geq 0$ such that $w_{\Gamma}=\sum_{\beta} n_{\beta} r^{\beta}$ is the $r$-adic expansion of $w_{\Gamma}$, and $\mathbf{c}_{\beta}=(1,1, \ldots, 1) \quad(\beta$ terms $)$. If $\Gamma \neq X \pm 1$, then $H_{\Gamma} \simeq \mathrm{GL}\left(e_{\Gamma} w_{\Gamma}, \varepsilon_{\Gamma} q^{\delta_{\Gamma}}\right)$ and $D(\Gamma)=\prod_{\beta}\left(R_{m_{\Gamma}, \alpha_{\Gamma}, 0, \mathbf{c}_{\beta}}\right)^{n_{\beta}}$, where $n_{\beta}$ and $\mathbf{c}_{\beta}$ defined as before.

Fix $1 \leq i \leq t$. If $\left(D_{i}, \theta_{i}\right)$ is of type $\Gamma$, then $D_{i}$ is a component of $D(\Gamma)$, and so $D_{i}=R_{m_{\Gamma}, \alpha_{\Gamma}, 0, c_{\beta}}$. Suppose for the same $i,\left(D_{i}, \theta_{i}^{\prime}\right)$ is of type $\Gamma^{\prime}$. Thus $D_{i}$ is also a component of $D^{\prime}\left(\Gamma^{\prime}\right)$, and hence $D_{i}=R_{m_{\Gamma^{\prime}}, \alpha_{\Gamma^{\prime}}, 0, \mathrm{c}_{\beta^{\prime}}}$. So $m_{\Gamma}=m_{\Gamma^{\prime}}, \alpha_{\Gamma}=\alpha_{\Gamma^{\prime}}$, and $\beta=\beta^{\prime}$. Since $D\left(\Gamma^{\prime}\right)$ and $D^{\prime}\left(\Gamma^{\prime}\right)$ are conjugate in $G$, there exists a component $D_{j}$ of $D\left(\Gamma^{\prime}\right)$, for $1 \leq j \leq t$, such that $\left(D_{j}, \theta_{j}\right)$ is of type $\Gamma^{\prime}$ and $D_{j}=R_{m_{\Gamma^{\prime}}, \alpha_{\Gamma^{\prime}}, 0, \mathrm{c}_{\beta^{\prime}}}$. So $D_{j}$ and $D_{i}$ have the same form $R_{m_{\Gamma}, \alpha_{\Gamma}, 0, \mathrm{c}_{\beta}}$. By $(2 \mathrm{E})$, there exists $h \in N_{G}(D)$ permuting $\left(V_{i}, D_{i}\right),\left(V_{j}, D_{j}\right)$ and holding $\left(V_{k}, D_{k}\right)$ fixed for $k \neq i, j$. Thus $h$ permutes $\left(D_{i}, \theta_{i}\right),\left(D_{j}, \theta_{j}\right)$ and holds $\left(D_{k}, \theta_{k}\right)$ fixed for $k \neq i, j$. Replacing $\left(D_{i}, \theta_{i}\right)$ by $\left(D_{i}, \theta_{i}\right)^{h}$, we may suppose both $\left(D_{i}, \theta_{i}\right)$ and $\left(D_{i}, \theta_{i}^{\prime}\right)$ are of the same type $\Gamma^{\prime}$, and we may suppose this for all $i \geq 1$. By $(3 \mathrm{~J})(\mathrm{b})$, for each $i, \theta_{i}^{g_{i}}=\theta_{i}^{\prime}$ for some $g_{i} \in N_{I\left(V_{i}\right)}\left(D_{i}\right)$ and then $\theta^{g}=\theta^{\prime}$ for some $g \in N_{G}(D)$. It follows that $B=b^{G}=b^{\prime G}=B^{\prime}$.

Conversely, suppose $B=B^{\prime}$. If $b^{G_{0}}=b^{\prime G_{0}}$, which occurs when $G$ is a symplectic group, then $s$ and $s^{\prime}$ are conjugate in $G_{0}^{*}$ by $(3 \mathrm{C})$.

Suppose $b^{G_{0}} \neq b^{\prime G_{0}}$. Then $G$ is an orthogonal group, and $\left(b^{G_{0}}\right)^{g}=b^{\prime G_{0}}$ for some $g \in G$ of determinant -1 . So $B$ covers exactly two blocks $b^{G_{0}}$ and $b^{\prime G_{0}}$ of $G_{0}$. Let $N_{0}(\theta)$ be the stabilizer of $\theta$ in $N_{G_{0}}(D)$. Then $\left(N(\theta): N_{0}(\theta)\right)=1$ 
or 2. If $\left(N(\theta): N_{0}(\theta)\right)=2$, then $\theta^{x}=\theta$ for some $x \in G$ of determinant -1 . So $\left(b^{G_{0}}\right)^{x}=b^{G_{0}}$ and thus $\left(b^{G_{0}}\right)^{g}=b^{G_{0}}$ for all $g \in G$. This is impossible. Thus $N(\theta)=N_{0}(\theta)$ and then $m_{X \pm 1}(s)=0$ by [12, (7B) and (7C)]. It follows that $C_{I\left(V^{*}\right)}(s)=C_{G_{0}^{*}}(s)$, so there exists $x \in I\left(V^{*}\right)$ of determinant -1 such that $s^{x}$ and $s$ are not conjugate in $G_{0}^{*}$. Let $D_{x}$ be a Sylow $r$-subgroup of $C_{G_{0}}\left(s^{x *}\right)$, and $y^{*} \in Z\left(D_{x}\right)$ primary. Thus $D_{x}$ and $D$ are conjugate in $G$, and $s^{x *} \in C_{G}\left(y^{*}\right) \simeq \mathrm{GL}\left(m, \varepsilon q^{e}\right)$ for some $m \geq 1$. Let $y$ be a primary element of a Sylow $r$-subgroup of $Z\left(C_{G}\left(y^{*}\right)^{*}\right)$. Then $C_{G_{0}^{*}}(y)=C_{G}\left(y^{*}\right)^{*}$ and $\langle y\rangle$ is conjugate in $I\left(V^{*}\right)$ with the subgroup generated by a dual of $y^{*}$, so $y^{k}$ is a dual of $y^{*}$ for some integer $k \geq 1$ and $\langle y\rangle=\left\langle y^{k}\right\rangle$ by $\left|y^{k}\right|=r^{a}$. By the remark of (3E) we may suppose $s^{x}$ lies in the $r$-section containing $y^{k}$ and $s^{x} \in C_{G}\left(y^{*}\right)^{*}$. There exists a block $b_{x}$ of $C_{G_{0}}\left(y^{*}\right)$ labeled by $\left(s^{x},-\right)$, so that $\left(\left\langle y^{*}\right\rangle, b_{x}\right)$ is a Brauer pair of $G_{0}$ labeled by $\left(\left\langle y^{*}\right\rangle, s^{x},-\right)$ and $b_{x}^{G_{0}} \subseteq \mathscr{E}_{r}\left(G_{0},\left(s^{x}\right)\right)$ by $(3 \mathrm{C})$. Since $s$ and $s^{x}$ are conjugate in $I\left(V^{*}\right)$, it follows that $b^{G}=B=b_{x}^{G}$ by the first half of the proof and so $B$ covers $b_{x}^{G_{0}}$. Since $s$ and $s^{x}$ are not conjugate in $G_{0}^{*}$, it follows that $b^{G_{0}} \neq b_{x}^{G_{0}}$ and so $b_{x}^{G_{0}}=b^{\prime G_{0}}$. Thus $s^{x}$ and $s^{\prime}$ are conjugate in $G_{0}^{*}$. This completes the proof.

\section{WEIGHTS FOR CLASSICAL GROUPS}

In this section we count the number of $B$-weights for a block $B$ of finite classical groups. Given $\Gamma \in \mathscr{F}^{\prime}$ and integer $d \geq 0$, let $V_{\Gamma, d}$ be a unitary space of dimension $r^{d} e_{\Gamma} d_{\Gamma}$ over $\mathbb{F}_{q^{2}}$, or a symplectic or orthogonal space over $\mathbb{F}_{q}$ given by (3.12). Denote $G=G_{0}=\mathrm{U}\left(V_{\Gamma, d}\right)$ in the case $V_{\Gamma, d}$ is unitary, and $G=I\left(V_{\Gamma, d}\right), G_{0}=I_{0}\left(V_{\Gamma, d}\right)$ in the remaining cases. Let $0 \leq \gamma \leq d$, and $\mathbf{c}=\left(c_{1}, c_{2}, \ldots, c_{l}\right)$ a sequence of nonnegative integers such that $d-\gamma=$ $c_{1}+c_{2}+\cdots+c_{l}$. In addition, let

$$
R=R_{m_{\Gamma}, \alpha_{\Gamma}, \gamma}\left\{A_{c_{1}} \mid A_{c_{2}} \nmid \cdots\right\} A_{c_{l}},
$$

be a basic subgroup of $G, C=C_{G}(R)$, and $N=N_{G}(R)$. Then $C=C_{\Gamma} \otimes I_{\gamma} \otimes I_{\mathfrak{c}}$, where $I_{\gamma}$ and $I_{\mathrm{c}}$ are identity matrices of orders $r^{\gamma}$ and $r^{c_{1}+c_{2}+\cdots+c_{l}}$ respectively. Define $\theta$ on $C$ by $\theta\left(c \otimes I_{\gamma} \otimes I_{\mathrm{c}}\right)=\theta_{\Gamma}(c)$ for $c \in C_{\Gamma}$. Then $\theta$ is an irreducible character of $C$ and $(R, \theta)$ is of type $\Gamma$. Regard $\theta$ as a character of $C R$ trivial on $R$. Then the block $b$ of $C R$ containing $\theta$ has defect group $R$ and the Brauer pair $(R, b)$ of $G_{0}$ has label $\left(R, x_{\Gamma},-\right)$, where $b$ is regarded as a block of $C$, and $x_{\Gamma}=r^{d} e_{\Gamma} \Gamma$ in the case $G$ is unitary and $x_{\Gamma}$ is given by (3.14) in the remaining cases. Let $V_{m_{\Gamma}, \alpha_{\Gamma}, \gamma}$ be the underlying space of $R_{m_{\Gamma}, \alpha_{\Gamma}, \gamma}$, $G_{m_{\Gamma}, \alpha_{\Gamma}, \gamma}=\mathrm{U}\left(V_{m_{\Gamma}, \alpha_{\Gamma}, \gamma}\right)$ in the case $V_{m_{\Gamma}, \alpha_{\Gamma}, \gamma}$ is unitary, or $I\left(V_{m_{\Gamma}, \alpha_{\Gamma}, \gamma}\right)$ in the remaining case. If $\theta_{\Gamma} \otimes I_{\gamma}$ is the character of $C_{G_{m_{\Gamma}, \alpha_{\Gamma}, \gamma}}\left(R_{m_{\Gamma}, \alpha_{\Gamma}, \gamma}\right)=C_{\Gamma} \otimes I_{\gamma}$ defined by $\left(\theta_{\Gamma} \otimes I_{\gamma}\right)\left(c \otimes I_{\gamma}\right)=\theta_{\Gamma}(c)$ for $c \in C_{\Gamma}$ and $N\left(\theta_{\Gamma} \otimes I_{\gamma}\right)$ is its stabilizer in $N_{G_{m_{\Gamma}, \alpha_{\Gamma}, \gamma}}\left(R_{m_{\Gamma}, \alpha_{\Gamma}, \gamma}\right)$, then by (2.2) or (2.5)

$$
\begin{aligned}
N(\theta) & =\left(N\left(\theta_{\Gamma} \otimes I_{\gamma}\right) / R_{m_{\Gamma}, \alpha_{\Gamma}, \gamma}\right) \otimes N_{\mathbf{S}(u)}\left(A_{\mathbf{c}}\right), \\
N(\theta) / R & \simeq\left(N\left(\theta_{\Gamma} \otimes I_{\gamma}\right) / R_{m_{\Gamma}, \alpha_{\Gamma}, \gamma}\right) \times \operatorname{GL}\left(c_{1}, r\right) \times \cdots \times \mathrm{GL}\left(c_{l}, r\right) .
\end{aligned}
$$

Thus the characters $\psi$ in $\operatorname{Irr}^{0}(N(\theta), \theta)$ are parametrized by $(l+1)$-tuples $\left(\psi_{0}, \psi_{1}, \ldots, \psi_{l}\right)$, where $\psi_{0} \in \operatorname{Irr}^{0}\left(N\left(\theta_{\Gamma} \otimes I_{\gamma}\right), \theta_{\Gamma} \otimes I_{\gamma}\right)$ and $\psi_{i}$ is an irreducible character of $\operatorname{GL}\left(c_{i}, r\right)$ of defect 0 for $i \geq 1$. Necessarily, $\psi_{i}$ are one of the $r-1$ Steinberg characters of $\mathrm{GL}\left(c_{i}, r\right)$ for $i \geq 1$. By (3A) or (3I) there are 
$\beta_{\Gamma} e_{\Gamma}$ such characters $\psi_{0}$, so that there are $\beta_{\Gamma} e_{\Gamma}(r-1)^{l}$ such characters $\psi$, where $\beta_{\Gamma}=1$ or 2 according as $\Gamma \in \mathscr{F}_{1} \cup \mathscr{F}_{2}$ or $\Gamma \in \mathscr{F}_{0}$. Thus there are $\beta_{\Gamma} e_{\Gamma}(r-1)^{l} b^{G}$-weights of the form $(R, I(\psi))$.

(4A). Let $V=V_{\Gamma, d}, B$ a block of $G$ with defect group $D$ and root block b such that $[V, D]=V$ and $\mathbf{b}^{G_{0}} \subseteq \mathscr{E}_{r}\left(G_{0},\left(x_{\Gamma}\right)\right)$. Then there are exactly $\beta_{\Gamma} e_{\Gamma} r^{d}$ $B$-weights $(R, \varphi)$, where $R$ runs over the basic subgroups of $G$ with degree $\beta_{\Gamma} e_{\Gamma} d_{\Gamma} r^{d}$.

Proof. (1) Suppose $R=R_{m, \alpha, \gamma, \mathrm{c}}$ is a basic subgroup of $G,(R, \varphi)$ is a $B$ weight, and $\varphi$ covers the irreducible character $\theta$ of $C_{G}(R) R$. Then the block $b$ of $C_{G}(R) R$ containing $\theta$ has a defect group $R$ and $b^{G}=B$. By (3B) or (3J)(a) $(R, \theta)$ has type $\Delta$ for some $\Delta \in \mathscr{F}^{\prime}$ and $(R, b)$ has label $\left(R, x_{\Delta},-\right)$, where $b$ is regarded as a block of $C_{G}(R)$. If $V$ is unitary, then $\Delta=\Gamma$ by [7, (3.2)]. Suppose $V$ is a symplectic or orthogonal space. Let $\left(D^{\prime}, \mathbf{b}^{\prime}\right)$ be a maximal pair containing $(R, b)$, so that $\mathbf{b}^{\prime} G=B$. As a block of $C_{G}\left(D^{\prime}\right) D^{\prime}, \mathbf{b}^{\prime}$ is also a root block of $B$ and $b^{G_{0}}=\mathbf{b}^{\prime G_{0}} \subseteq \mathscr{E}_{r}\left(G_{0},\left(x_{\Delta}\right)\right)$ by (3C). Since $D^{\prime}$ is a defect group of $B, D^{\prime}$ and $D$ are conjugate in $G$ and so $\left[V, D^{\prime}\right]=V$. By (3K) $x_{\Delta}$ and $x_{\Gamma}$ are conjugate in $I\left(V^{*}\right)$, where $V^{*}$ is the underlying space of $G_{0}^{*}$. Thus $\Delta=\Gamma$ and $m=m_{\Gamma}, \alpha=\alpha_{\Gamma}, \gamma+c_{1}+c_{2}+\cdots+c_{l}=d$.

The number of different sequences $\mathbf{c}=\left(c_{1}, c_{2}, \ldots, c_{l}\right)$ such that

$$
d\left(R_{m_{\Gamma}, \alpha_{\Gamma}, \gamma, \mathrm{c}}\right)=\beta_{\Gamma} e_{\Gamma} d_{\Gamma} r^{d} \text { and } l\left(R_{m_{\Gamma}, \alpha_{\Gamma}, \gamma, \mathrm{c}}\right)=l
$$

is $\left(\begin{array}{c}d-\gamma-1 \\ l-1\end{array}\right)$. Here $1 \leq l \leq d-\gamma$ when $d-\gamma \geq 1 ; l=0$ when $d=\gamma$, and $\left(\begin{array}{c}-1 \\ -1\end{array}\right)$ is interpreted as 1 . There are $\beta_{\Gamma} e_{\Gamma}(r-1)^{l}$ characters $\varphi$ associated with $R_{m_{\Gamma}, \alpha_{\Gamma}, \gamma, \mathrm{c}}$, so that there are

$$
\beta_{\Gamma} e_{\Gamma} \sum_{\gamma=0}^{d} \sum_{l=0}^{d-\gamma}\left(\begin{array}{c}
d-\gamma-1 \\
l-1
\end{array}\right)(r-1)^{l}=\beta_{\Gamma} e_{\Gamma} r^{d},
$$

characters associated with $R_{m_{\Gamma}, \alpha_{\Gamma}, \gamma, \mathrm{c}}$ 's.

(2) Suppose $V$ is a symplectic or orthogonal space. By $(3 \mathrm{~J})(\mathrm{b})$ the pair $(R, \theta)$ of type $\Gamma$ is determined uniquely up to conjugacy in $N_{G}(R)$, so that there are $\beta_{\Gamma} e_{\Gamma} r^{d} B$-weights $(R, \varphi)$. Suppose $V$ is a unitary space and $\left(R, b^{\prime}\right)$ is another Brauer pair of $G$ such that $b^{\prime G}=B$, and $\theta^{\prime}$ is the canonical character of $b^{\prime}$, where $R=R_{m_{\Gamma}, \alpha_{\Gamma}, \gamma, \mathrm{c}}$. Then $\left(R, \theta^{\prime}\right)$ has type $\Gamma, C=C_{G}(R)=$ $C_{\Gamma} \otimes I_{\gamma} \otimes I_{\mathbf{c}}$, and $\theta^{\prime}$ has the form $\theta_{\Gamma}^{\prime} \otimes I_{\gamma} \otimes I_{\mathbf{c}}$, where $\theta_{\Gamma}^{\prime}$ is an irreducible character of $C_{\Gamma}$. If $b_{\Gamma}^{\prime}$ is the block of $C_{\Gamma}$ containing $\theta_{\Gamma}^{\prime}$, then $b_{\Gamma}^{\prime G_{\Gamma}}=B_{\Gamma}$ and both $B_{\Gamma}$ and $b_{\Gamma}^{\prime}$ have a defect group $R_{\Gamma}$. By definition $b_{\Gamma}^{G_{\Gamma}}=B_{\Gamma}$ and $b_{\Gamma}$ has a defect group $R_{\Gamma}$. Thus $b_{\Gamma}^{w}=b_{\Gamma}^{\prime}$ for some $w \in N_{\Gamma}$ by Brauer First Main Theorem. A similar proof to that of $(3 \mathrm{~J})(\mathrm{b})$ shows that $\theta^{\prime}=\theta^{n}$ for some $n \in N_{G}(R)$. Thus (4A) follows in this case.

Remark. In the notation of (4A), suppose $V$ is orthogonal, $G=I(V)$, and $G_{0}=I_{0}(V)$. If $(R, \theta)$ has type $\Gamma$, then $\left|N(\theta): N_{0}(\theta)\right|=\beta_{\Gamma}$ and for each $\psi \in \operatorname{Irr}^{0}(N(\theta), \theta)$, the restriction $\left.\psi\right|_{N_{0}(\theta)}$ of $\psi$ to $N_{0}(\theta)$ is irreducible, where $N_{0}(\theta)=N(\theta) \cap G_{0}$. Indeed in the notation above $\psi=\left(\psi_{0}, \psi_{1}, \ldots, \psi_{l}\right)$ as a character of $N(\theta) / R$, where $\psi_{0} \in \operatorname{Irr}^{0}\left(N\left(\theta_{\Gamma} \otimes I_{\gamma}\right), \theta_{\Gamma} \otimes I_{\gamma}\right)$, and $\psi_{i}$ is an irreducible character of $\mathrm{GL}\left(c_{i}, r\right)$ of defect 0 for $i \geq 1$. Let $N_{0}\left(\theta_{\Gamma} \otimes I_{\gamma}\right)$ be the subgroup of $N\left(\theta_{\Gamma} \otimes I_{\gamma}\right)$ of determinant 1. Then $\left|N\left(\theta_{\Gamma} \otimes I_{\gamma}\right): N_{0}\left(\theta_{\Gamma} \otimes I_{\gamma}\right)\right|=\beta_{\Gamma}$ 
and the restriction of $\psi_{0}$ to $N_{0}\left(\theta_{\Gamma} \otimes I_{\gamma}\right)$ is irreducible by the remark of (3I). Thus by (4.1) $\left|N(\theta): N_{0}(\theta)\right|=\beta_{\Gamma}$. Now the restriction of $\psi$ to

$$
H=\left(N_{0}\left(\theta_{\Gamma} \otimes I_{\gamma}\right) / R_{m_{\Gamma}, \alpha_{\Gamma}, \gamma}\right) \times \operatorname{GL}\left(c_{1}, r\right) \times \operatorname{GL}\left(c_{2}, r\right) \times \cdots \times \operatorname{GL}\left(c_{l}, r\right)
$$

is irreducible. Since $N_{0}(\theta) / R \geq H,\left.\psi\right|_{N_{0}(\theta) / R}$ is irreducible, and so $\left.\psi\right|_{N_{0}(\theta)}$ is irreducible.

Given $\Gamma \in \mathscr{F}^{\prime}$ and integer $w_{\Gamma} \geq 1$, let $G=\mathrm{U}(V)$ or $I(V)$ and $G_{0}=G$ or $I_{0}(V)$, where in the former case $V$ is a unitary space of dimension $w_{\Gamma} e_{\Gamma} d_{\Gamma}$ over $\mathbb{F}_{q^{2}}$, in the latter case $V$ is a symplectic or orthogonal space over $\mathbb{F}_{q}$ such that $\operatorname{dim} V=w_{\Gamma} \beta_{\Gamma} e_{\Gamma} d_{\Gamma}$ and if $V$ is orthogonal, then $\eta(V)=\varepsilon^{w_{\Gamma}}$ or $\varepsilon_{\Gamma}^{w_{\Gamma} e_{\Gamma}}$ according as $\Gamma \in \mathscr{F}_{0}$ or $\Gamma \in \mathscr{F}_{1} \cup \mathscr{F}_{2}$. Thus if $V$ is unitary, then $s=w_{\Gamma} e_{\Gamma} \Gamma$ is a semisimple element of $G$ and $C_{G}(s) \simeq \mathrm{GL}\left(w_{\Gamma} e_{\Gamma}, \varepsilon_{\Gamma} q^{\delta_{\Gamma}}\right)$, so that $G$ has a block $B$ labeled by $(s,-)$ and a defect group $D$ of $B$ acts fixed-point freely on $V$ since we may suppose $D$ is a Sylow $r$-subgroup of $C_{G}(s)$. In the remaining case, a semisimple element $s^{*}$ in $G_{0}$ exists such that $m_{\Gamma}\left(s^{*}\right)=\beta_{\Gamma} e_{\Gamma} w_{\Gamma}$ and $\eta_{\Gamma}\left(s^{*}\right)=\eta(V)$, so that a primary element of a Sylow $r$-subgroup of $C_{G}\left(s^{*}\right)$ exists and by the remark of (3E) a dual $s$ of $s^{*}$ exists in $G_{0}^{*}$ which is uniquely determined in $I\left(V^{*}\right)$ up to conjugacy, where $V^{*}$ is the underlying space of $G_{0}^{*}$. Moreover, by (3K) $s$ uniquely determines a block $B$ of $G$ which covers a block in $\mathscr{E}_{r}\left(G_{0},(s)\right)$ and whose defect group acts fixed-point freely on $V$.

For each $\Gamma \in \mathscr{F}^{\prime}$ and integer $d \geq 0$, let $\mathscr{C}_{\Gamma, d}=\left\{\varphi_{\Gamma, d, i, j}: 1 \leq i \leq\right.$ $\left.\beta_{\Gamma} e_{\Gamma}, 1 \leq j \leq r^{d}\right\}$ be the set of characters associated with basic subgroups of $G=\mathrm{U}\left(V_{\Gamma, d}\right)$ or $I\left(V_{\Gamma, d}\right)$ in $(4 \mathrm{~A})$.

(4B). With the preceding notation, let $B$ be a block of $G$ with defect group $D$ and root block b such that $[V, D]=V$ and $\mathbf{b}^{G_{0}} \subseteq \mathscr{E}_{r}\left(G_{0},(s)\right)$. Then the number of $B$-weights is the number $f_{\Gamma}$ of assignments

$$
\coprod_{d \geq 0} \mathscr{C}_{\Gamma, d} \rightarrow\{r \text {-cores }\}, \quad \varphi_{\Gamma, d, i, j} \mapsto \kappa_{\Gamma, d, i, j}
$$

such that

$$
\sum_{d \geq 0} r^{d} \sum_{i=1}^{\beta_{\Gamma} e_{\Gamma}} \sum_{j=1}^{r^{d}}\left|\kappa_{\Gamma, d, i, j}\right|=w_{\Gamma}
$$

Proof. Let $(R, \varphi)$ be a $B$-weight of $G, C=C_{G}(R)$, and $N=N_{G}(R)$. Then there exists a block $b$ of $C R$ with defect group $R$ such that $b^{G}=B$ and $\varphi \in b^{N}$. We may suppose $Z(D) \leq Z(R) \leq R \leq D$. Let $z$ be a primary element of $D$ defined by the remark of $(2 \mathrm{D})$. Then $z \in Z(D)$ and $[V, z]=V$, so that $C_{V}(R)=0$. Thus in the decomposition (2B) or (2D) of $R$, we may suppose

$$
R=R_{1}^{d_{1}} \times R_{2}^{d_{2}} \times \cdots \times R_{u}^{d_{u}},
$$

where $R_{i}$ 's are distinct nontrivial basic subgroups and $R_{i}$ appears $d_{i}$ times as a component of $R$. Let $V_{i}$ be the underlying space of $R_{i}, G_{i}=\mathrm{U}\left(V_{i}\right)$ or $I\left(V_{i}\right)$ according as $V_{i}$ is or is not a unitary space, $C_{i}=C_{G_{i}}\left(R_{i}\right)$, and $N_{i}=N_{G_{i}}\left(R_{i}\right)$. Then $C=C_{1}^{d_{1}} \times C_{2}^{d_{2}} \times \cdots \times C_{u}^{d_{u}}$. Let $\theta$ be the canonical character of $b$, so that we may suppose $\theta=\prod_{i=1}^{u} \theta_{i}^{d_{i}}$, where $\theta_{i}$ is an irreducible character of $C_{i} R_{i}$ trivial on $R_{i}$. Let $z_{i}$ be the restriction of $z$ on $V_{i}$ and $K_{i}=C_{G_{i}}\left(z_{i}\right)$ for all $i$. Then $K_{j}$ and $\prod_{i=1}^{u} K_{i}^{d_{i}}$ are a regular subgroup of $I_{0}\left(V_{j}\right)$ and $G_{0}$, 
so that $\prod_{i=1}^{u}\left(K_{i}^{*}\right)^{d_{i}}$ is embedded as a regular subgroup of $G_{0}^{*}$. If $\left(R_{i}, s_{i},-\right)$ is a label of the Brauer pair $\left(R_{i}, \theta_{i}\right)$, then $s_{i} \in K_{i}^{*}, \prod_{i=1}^{u} s_{i}^{d_{i}} \in \prod_{i=1}^{u}\left(K_{i}^{*}\right)^{d_{i}}$, and so $\left(R, \prod_{i=1}^{u} s_{i}^{d_{i}},-\right)$ is a label of the Brauer pair $(R, b)$. Thus $s_{i}$ and $x_{\Gamma}$ are conjugate in $I_{0}\left(V_{i}\right)^{*},\left(R_{i}, \theta_{i}\right)$ has type $\Gamma$, and $R_{i}=R_{m_{\Gamma}, \alpha_{\Gamma}, \gamma_{i}, \mathbf{c}_{i}}$ for some $\gamma_{i}$ and $\mathbf{c}_{i}$. It is clear that

$$
N(\theta)=\prod_{i=1}^{u} N\left(\theta_{i}\right) \imath \mathbf{S}\left(d_{i}\right),
$$

where $N\left(\theta_{i}\right)$ is the stabilizer of $\theta_{i}$ in $N_{i}$. In particular, if $\psi \in \operatorname{Irr}^{0}(N(\theta), \theta)$, then $\psi=\prod_{i=1}^{u} \psi_{i}$, where $\psi_{i}$ is an irreducible character of $N\left(\theta_{i}\right) l \mathbf{S}\left(d_{i}\right)$ covering $\theta_{i}^{d_{i}}$. Moreover, $\psi_{i}$ has defect 0 as a character of

$$
N\left(\theta_{i}\right) \prec \mathbf{S}\left(d_{i}\right) / R_{i}^{d_{i}} \simeq\left(N\left(\theta_{i}\right) / R_{i}\right) \prec \mathbf{S}\left(d_{i}\right) .
$$

Let $\operatorname{Irr}^{0}\left(N\left(\theta_{i}\right), \theta_{i}\right)=\left\{\varphi_{i, j}: 1 \leq j \leq \beta_{\Gamma} e_{\Gamma}(r-1)^{l\left(R_{i}\right)}\right\}$. As shown in the proof of [3, (2C)], the irreducible characters of defect 0 of $\left(N\left(\theta_{i}\right) / R_{i}\right) \imath \mathbf{S}\left(d_{i}\right)$ covering $\theta_{i}^{d_{i}}$ are in bijection with assignments $\varphi_{i, j} \mapsto \kappa_{i, j}$ of characters to $r$-cores such that $\sum_{j \geq 1}\left|\kappa_{i, j}\right|=d_{i}$. Thus the irreducible characters of $\operatorname{Irr}^{0}(N(\theta), \theta)$ are in bijection with assignments $\varphi_{i, j} \mapsto \kappa_{i, j}$ of characters to $r$-cores such that

$$
\sum_{i=1}^{u}\left(\operatorname{deg} R_{i}\right) \sum_{j \geq 1}\left|\kappa_{i, j}\right|=\beta_{\Gamma} e_{\Gamma} d_{\Gamma} w_{\Gamma} .
$$

For fixed $d \geq 0$, the number of irreducible characters associated with basic groups of degree $\beta_{\Gamma} e_{\Gamma} d_{\Gamma} r^{d}$ is $\beta_{\Gamma} e_{\Gamma} r^{d}$. Let $\mathscr{C}_{\Gamma, d}=\left\{\varphi_{\Gamma, d, i, j}: 1 \leq i \leq \beta_{\Gamma} e_{\Gamma}\right.$, $\left.1 \leq j \leq r^{d}\right\}$ be the set of these characters. Then the number of $B$-weights is the number of assignments

$$
\coprod_{d \geq 0} \mathscr{C}_{\Gamma, d} \rightarrow\{r \text {-cores }\}, \quad \varphi_{\Gamma, d, i, j} \mapsto \kappa_{\Gamma, d, i, j},
$$

such that

$$
\sum_{d \geq 0} \beta_{\Gamma} e_{\Gamma} r^{d} d_{\Gamma} \sum_{i=1}^{\beta_{\Gamma} e_{\Gamma}} \sum_{j=1}^{r^{d}}\left|\kappa_{\Gamma, d, i, j}\right|=\beta_{\Gamma} e_{\Gamma} d_{\Gamma} w_{\Gamma} .
$$

This induces the required condition of (4B).

(4C). With the preceding notation, let $G=\mathrm{O}(V)$ be an orthogonal group, $G_{0}=$ $\mathrm{SO}(V)$, and $R$ a radical subgroup of $G$ such that $[V, R]=V$. Let $(R, b)$ a Brauer pair of $G_{0}$ labeled by $(R, s,-)$ and $\theta$ the canonical character of $b$. Then $\left|N(\theta): N_{0}(\theta)\right|=\beta_{\Gamma}$ and the restriction $\left.\psi\right|_{N_{0}(\theta)}$ of each $\psi \in \operatorname{Irr}^{0}(N(\theta), \theta)$ to $N_{0}(\theta)$ is irreducible, where $N_{0}(\theta)=N(\theta) \cap G_{0}$.

Proof. In the notation above $R=R_{1}^{d_{1}} \times R_{2}^{d_{2}} \times \cdots \times R_{u}^{d_{u}}, V_{i}$ is the underlying space of $R_{i}, C=C_{G}(R)=\prod_{i=1}^{u} C_{i}^{d_{i}}$, and $\theta=\prod_{i=1}^{u} \theta_{i}^{d_{i}}$, where $\theta_{i}$ is an irreducible character of $C_{i}=C_{\mathrm{O}\left(V_{i}\right)}\left(R_{i}\right)$ for $i \geq 1$. Each $\left(R_{i}, \theta_{i}\right)$ has type $\Gamma$. Let $N\left(\theta_{i}\right)$ and $N_{0}\left(\theta_{i}\right)$ be the stabilizers of $\theta_{i}$ in $N_{\mathrm{O}\left(V_{i}\right)}\left(R_{i}\right)$ and $N_{\mathrm{SO}\left(V_{i}\right)}\left(R_{i}\right)$ respectively. By the remark of $(4 \mathrm{~A}),\left|N\left(\theta_{i}\right): N_{0}\left(\theta_{i}\right)\right|=\beta_{\Gamma}$ and so $\left|N(\theta): N_{0}(\theta)\right|=\beta_{\Gamma}$ since $N(\theta)=\prod_{i=1}^{u} N\left(\theta_{i}\right)$ $\mathbf{S}\left(d_{i}\right)$. If $\psi \in \operatorname{Irr}^{0}(N(\theta), \theta)$, then $\psi=\prod_{i=1}^{u} \psi_{i}$, where $\psi_{i}$ is an irreducible character of $N\left(\theta_{i}\right) \backslash \mathbf{S}\left(d_{i}\right)$ covering $\theta_{i}^{d_{i}}$. Moreover, 
$\psi_{i}$ has defect 0 as a character of $N\left(\theta_{i}\right)$ $\mathbf{S}\left(d_{i}\right) / R_{i}^{d_{i}} \simeq\left(N\left(\theta_{i}\right) / R_{i}\right)$ $\mathbf{S}\left(d_{i}\right)$. Let $N_{0}\left(\theta_{i}^{d_{i}}\right)$ be the subgroup of $N\left(\theta_{i}\right)$ 乙 $\mathbf{S}\left(d_{i}\right)$ of determinant 1 . It then suffices to show that the restriction of $\psi_{i}$ to $N_{0}\left(\theta_{i}^{d_{i}}\right)$ is irreducible. Thus we may suppose $u=1$ and $d=d_{1}$, so that $\theta=\theta_{1}^{d}$ and $N(\theta)=N\left(\theta_{1}\right) \imath \mathbf{S}(d)$. Since $\left|N(\theta): N_{0}(\theta)\right| \leq 2,\left.\psi\right|_{N_{0}(\theta)}$ is irreducible if and only if $N(\theta)$ stabilizes an irreducible constituent of $\left.\psi\right|_{N_{0}(\theta)}$.

Let $T=N\left(\theta_{1}\right), H=N(\theta)=T \backslash \mathbf{S}(d), X=T^{d}$ the base subgroup of $H$, $H_{0}=N_{0}(\theta)$, and $X_{0}$ the subgroup of $X$ of determinant 1. Then $H=X \rtimes \mathbf{S}(d)$ and $H_{0}=X_{0} \rtimes \mathbf{S}(d)$. We may suppose $\left|H: H_{0}\right|=2$ and hence $\left|T: T_{0}\right|=2$, where $T_{0}=T \cap I_{0}\left(V_{1}\right)$. Morever, $\left(R_{1}, \theta_{1}\right)$ has type $\Gamma$ and the restriction of each character in $\operatorname{Irr}^{0}\left(T, \theta_{1}\right)$ to $T_{0}$ is irreducible by the remark of $(4 \mathrm{~A})$. As shown in the proof of $[3,(2 \mathrm{~B})]$ (cf. also [17, 5.20]), the irreducible characters of $H$ can be obtained as follows: Let $\operatorname{Irr} T=\left\{\xi^{1}, \xi^{2}, \ldots, \xi^{t}\right\}$ be the complete set of irreducible characters of $T$, and $\xi$ an irreducible character of $X$. Then $\mathbf{m}=\left(m_{1}, m_{2}, \ldots, m_{t}\right)$ is called the type of $\xi$ if $m_{i}$ is the multiplicity of $\xi^{i}$ as a factor of $\xi$. The stabilizer of $\xi$ in $H$ is $X \mathbf{S}_{\mathbf{m}}$, and $\xi$ can be extended to an irreducible character $\tilde{\xi}$ of $X \mathbf{S}_{\mathbf{m}}$ (see $[17,5.13]$ ), where $\mathbf{S}_{\mathbf{m}}$ is the Young subgroup of $\mathbf{S}(d)$ of type $\mathbf{m}$. By Clifford theory, all irreducible characters of $X \mathbf{S}_{\mathbf{m}}$ covering $\xi$ have form $\tilde{\xi} \zeta$ and $\operatorname{Ind}_{X \mathrm{~S}_{\mathbf{m}}}^{H}(\tilde{\xi} \zeta)$ is irreducible, where $\zeta$ is an irreducible character of $X \mathbf{S}_{\mathrm{m}}$ trivial on $X$. Moreover, these characters $\left\{\operatorname{Ind}_{X \mathbf{S}_{\mathbf{m}}}^{H}(\tilde{\xi} \zeta)\right\}$ consist of a complete set of irreducible characters of $H$ as $\xi$ runs over the representatives of conjugacy $H$-classes of $\operatorname{Irr} X$, and, while $\xi$ is fixed, $\zeta$ runs over irreducible characters of $\mathbf{S}_{\mathbf{m}}$, where $\mathbf{m}$ is the type of $\xi$ (see $[17,5.20])$. In particular, $\operatorname{Ind}_{X \mathrm{~S}_{m}}^{H}(\tilde{\xi} \zeta)$ has defect 0 as a character of $H / R$ if and only if $\zeta$ has defect 0 , and $\xi$ has defect 0 as a character of $X / R$. If $\operatorname{Ind}_{X \mathrm{~S}_{\mathrm{m}}}^{H}(\tilde{\xi} \zeta) \in \operatorname{Irr}^{0}(H, \theta)$, then we may suppose $\xi$ covers $\theta$.

Suppose $\xi \in \operatorname{Irr}^{0}(X, \theta)$. Then the restriction $\xi_{0}=\left.\xi\right|_{X_{0}}$ is irreducible since $\left.\xi\right|_{T_{0}^{d}}$ is irreducible by the remark of $(4 \mathrm{~A})$. Let $K$ be the stabilizer of $\xi_{0}$ in $H_{0}$. Then $X_{0} \mathbf{S}_{\mathrm{m}} \leq K$, where $\mathrm{m}$ is the type of $\xi$. We claim $X_{0} \mathbf{S}_{\mathbf{m}}=K$. Indeed if there exists $x \in K \backslash X_{0} \mathbf{S}_{\mathbf{m}}$, then we may suppose $x \in \mathbf{S}(d) \backslash \mathbf{S}_{\mathbf{m}}, \xi^{x} \neq \xi$, and $\left.\xi^{x}\right|_{0}=\xi_{0}$, since $H_{0}=X_{0} \mathbf{S}(d)$ and the stabilizer of $\xi$ is $X \mathbf{S}_{\mathbf{m}}$. In particular, $d>1$. Thus $\xi_{i} \neq \xi_{i}^{\prime}$ for some $i$ th components $\xi_{i}$ and $\xi_{i}^{\prime}$ of $\xi$ and $\xi^{x}$ respectively and so $\xi_{i}(h) \neq \xi_{i}^{\prime}(h)$ for some $h \in T$. Since $\left.\xi\right|_{X_{0}}=\left.\xi^{x}\right|_{X_{0}}, h$ has determinant -1 . Let $w=\operatorname{diag}\left\{w_{1}, w_{2}, \ldots, w_{d}\right\} \in X$ such that $w_{i}=h=w_{j}$ for some $j \neq i$, and $w_{k}=1$ for $k \neq i, j$. Then $w \in X_{0}$ and so $\xi(w)=\xi^{x}(w)$. But the $i$ th components of $\xi(w)$ and $\xi^{x}(w)$ are $\xi_{i}(h)$ and $\xi_{i}^{\prime}(h)$ respectively. This is impossible and the claim holds.

Since $\tilde{\xi}$ is an extension of $\xi$ to $X \mathbf{S}_{\mathbf{m}}$, it follows $\left.\tilde{\xi}\right|_{X_{0}}=\xi_{0}$ and hence $\left.\tilde{\xi}\right|_{X_{0} \mathbf{S}_{\mathbf{m}}}=\tilde{\xi}_{0}$ is an extension of $\xi_{0}$ to $X_{0} \mathbf{S}_{\mathbf{m}}$. By Clifford theory again, each irreducible character of $X_{0} \mathbf{S}_{\mathbf{m}}$ covering $\xi_{0}$ has the form $\tilde{\xi}_{0} \chi$, where $\chi$ is an irreducible character of $X_{0} \mathbf{S}_{\mathrm{m}}$ trivial on $X_{0}$, and each irreducible character of $H_{0}$ covering $\xi_{0}$ has the form $\operatorname{Ind}_{X_{0} \mathbf{S}_{\mathrm{m}}}^{H_{0}}\left(\tilde{\xi}_{0} \chi\right)$. Now for $\psi \in \operatorname{Irr}^{0}(H, \theta), \psi=$ $\operatorname{Ind}_{X \mathbf{S}_{\mathrm{m}}}^{H}(\tilde{\xi} \zeta)$ for some irreducible character $\xi$ of $X$ with defect 0 as a character of $X / R$, and $\left.\xi\right|_{X_{0}}=\xi_{0}$ is irreducible. Thus there is an irreducible constituent $\psi_{0}$ of $\left.\psi\right|_{H_{0}}$ covering $\xi_{0}$ and so $\psi_{0}=\operatorname{Ind}_{X_{0} \mathbf{s}_{\mathrm{m}}}^{H_{0}}\left(\tilde{\xi}_{0} \chi\right)$. We claim that $\psi_{0}^{\tau}=\psi_{0}$ for any $\tau \in X$. Indeed this is true for $\tau \in X_{0}$ and we may suppose $\tau$ has 
determinant -1 . Since $\left|X \mathbf{S}_{\mathbf{m}}: X_{0} \mathbf{S}_{\mathbf{m}}\right| \leq 2, \tau$ normalizes $X_{0} \mathbf{S}_{\mathbf{m}}$ and for $x, h \in$ $H_{0}$, we have $h^{\tau^{-1} x} \in X_{0} \mathbf{S}_{\mathbf{m}}$ if and only if $h^{x} \in X_{0} \mathbf{S}_{\mathbf{m}}$ since $h^{\tau^{-1} x}=h^{x\left(x^{-1} \tau^{-1} x\right)}$ and $x^{-1} \tau^{-1} x \in X$. If $h^{\tau^{-1} x} \in X_{0} \mathbf{S}_{\mathbf{m}}$, then $\left(\tilde{\xi}_{0} \chi\right)\left(h^{\tau^{-1} x}\right)=\left(\tilde{\xi}_{0} \chi\right)^{\tau^{\prime}}\left(h^{x}\right)$, where $\tau^{\prime}=x^{-1} \tau x \in X$. Since $\left.\tilde{\xi}\right|_{X_{0} \mathbf{S}_{\mathbf{m}}}=\tilde{\xi}_{0}$ is irreducible and $\chi$ is trivial on $X_{0}$, $\tilde{\xi}_{0}^{g}=\tilde{\xi}_{0}$ and $\chi^{g}=\chi$ for any $g \in X$. Therfore $\left(\tilde{\xi}_{0} \chi\right)^{\tau^{\prime}}\left(h^{x}\right)=\left(\tilde{\xi}_{0} \chi\right)\left(h^{x}\right)$ and so $\left(\tilde{\xi}_{0} \chi\right)\left(h^{\tau^{-1} x}\right)=\left(\tilde{\xi}_{0} \chi\right)\left(h^{x}\right)$, for any $h, x \in H_{0}$. Thus $\psi_{0}^{\tau}=\psi_{0}$ and so $\left.\psi\right|_{H_{0}}=\psi_{0}$ is irreducible. This proves $(4 C)$.

We now prove the main theorem of unitary groups.

(4D). Let $V$ be a unitary space over $\mathbb{F}_{q^{2}}, G=U(V), B$ be a block of $G$ with label $(s, \kappa), \Pi_{\Gamma} s(\Gamma)$ the primary decomposition of $s, \sum_{\Gamma} V(\Gamma)$ the corresponding orthogonal decomposition of $V$, and $w_{\Gamma}$ the integer such that $\operatorname{dim} V(\Gamma)-d_{\Gamma}\left|\kappa_{\Gamma}\right|=d_{\Gamma} e_{\Gamma} w_{\Gamma}$. Then the following hold:

(1) The number of B-weights of $G$ is $\Pi_{\Gamma} f_{\Gamma}$, where $f_{\Gamma}$ is given by (4B). In particular, $f_{\Gamma}$ is the number of $e_{\Gamma^{-}}$-tuples $\left(\kappa_{1}, \kappa_{2}, \ldots, \kappa_{e_{\Gamma}}\right)$ of partitions $\kappa_{i}$ such that $\sum_{i=1}^{e_{\Gamma}}\left|\kappa_{i}\right|=w_{\Gamma}$.

(2) The number of $B$-weights of $G$ is the number $l(B)$ of irreducible modular characters in $B$.

Proof. Let $R$ be a radical subgroup of $G$ and $V=V_{0} \perp V_{+}$, where $V_{0}=C_{V}(R)$ and $V_{+}=[V, R]$. Then $R=R_{0} \times R_{+}$, where $R_{0}=\left\langle 1_{V_{0}}\right\rangle$ and $R_{+} \leq \mathrm{U}\left(V_{+}\right)$. Let $C=C_{G}(R), N=N_{G}(R)$, so that $C=C_{0} \times C_{+}, N=N_{0} \times N_{+}$, where $C_{0}=N_{0}=\mathrm{U}\left(V_{0}\right), C_{+}=C_{\mathrm{U}\left(V_{+}\right)}\left(R_{+}\right)$and $N_{+}=N_{\mathrm{U}\left(V_{+}\right)}\left(R_{+}\right)$. Suppose $b$ is a block of $C R$ with defect group $R$ and $b^{G}=B$. Then $b=b_{0} \times b_{+}$, where $b_{0}$ is a block of $C_{0} R_{0}=\mathrm{U}\left(V_{0}\right)$ of defect 0 , and $b_{+}$is a block of $C_{+} R_{+}$with defect group $R_{+}$. The canonical character $\theta$ of $b$ decomposes as $\theta_{0} \times \theta_{+}$, where $\theta_{0}$ and $\theta_{+}$are the canonical characters of $b_{0}$ and $b_{+}$respectively. Thus $N(\theta)=N_{0} \times N\left(\theta_{+}\right)$, where $N\left(\theta_{+}\right)$is the stabilizer of $\theta_{+}$in $N_{+}$.

Suppose $(R, I(\psi))$ is a $B$-weight of $G$, for some $\psi \in \operatorname{Irr}^{0}(N(\theta), \theta)$. Clearly $\psi=\psi_{0} \times \psi_{+}$for character $\psi_{0}$ of $N_{0}$ and $\psi_{+} \in \operatorname{Irr}^{0}\left(N\left(\theta_{+}\right), \theta_{+}\right)$. Since $\psi_{0}$ is a character of $N_{0}=C_{0}$ covering $\theta_{0}$, it follows that $\psi_{0}=\theta_{0}$. The correspondence $(R, I(\psi)) \mapsto\left(R_{+}, I_{+}\left(\psi_{+}\right)\right)$, where $\psi=\theta_{0} \times \psi_{+}$and $I_{+}\left(\psi_{+}\right)=$ $\operatorname{Ind}_{N\left(\theta_{+}\right)}^{N_{+}}\left(\psi_{+}\right)$, is clearly a bijection from $\left\{(R, I(\psi)): \psi \in \operatorname{Irr}^{0}(N(\theta), \theta)\right\}$ to $\left\{\left(R_{+}, I_{+}\left(\psi_{+}\right)\right): \psi_{+} \in \operatorname{Irr}^{0}\left(N\left(\theta_{+}\right), \theta_{+}\right)\right\}$.

By a theorem of Broué-Puig, [7, 3.2], we may suppose $s=s_{0} \times s_{+}$such that $s_{0} \in C_{0}, s_{+} \in C_{+},\left(s_{0}, \kappa\right)$ is the label of $b_{0}$, and $\left(s_{+},-\right)$is the label of $b_{+}^{\mathrm{U}\left(V_{+}\right)}$. In the correspondence above, $\left(R_{+}, I_{+}\left(\psi_{+}\right)\right)$is a $b_{+}^{\mathrm{U}\left(V_{+}\right)}$-weight. So the number of $B$-weights in $G$ is the number of $b_{+}^{\mathrm{U}\left(V_{+}\right)}$-weights in $\mathrm{U}\left(V_{+}\right)$. Thus we may suppose $V=V_{+}$.

Let $R=\prod_{i=1}^{t} R_{i}$ and $V=\bigoplus_{i=1}^{t} V_{i}$ be the decompositions of (2B), and let $C=\prod_{i=1}^{t} C_{i}$ and $\theta=\prod_{i=1}^{t} \theta_{i}$, where $C_{i}=C_{\mathrm{U}\left(V_{i}\right)}\left(R_{i}\right)$ and $\theta_{i}$ is a character of $C_{i}$. Since the block $b_{i}$ of $C_{i} R_{i}$ containing $\theta_{i}$ has a defect group $R_{i}$, $\left(R_{i}, \theta_{i}\right)$ has type $\Gamma$ for a unique $\Gamma \in \mathscr{F}^{\prime}$ by (3B). Moreover, if $\left(R_{i}, t_{i},-\right)$ is the label of $\left(R_{i}, b_{i}\right)$, then $\left(R, \prod_{i} t_{i},-\right)$ is the label of Brauer pair $(R, b)$ of $G$, where $b_{i}$ and $b$ are regarded as blocks of $C_{i}$ and $C$ respectively. By [7, (3.2)] $(R, s,-)$ is also a label of $(R, b)$, so that $s$ and $\prod_{i} t_{i}$ are conjugate in $G$. Let $R(\Gamma)=\prod_{i} R_{i}, C(\Gamma)=\prod_{i} C_{i}, \theta(\Gamma)=\prod_{\Gamma} \theta_{i}$, and $t(\Gamma)=\prod_{i} t_{i}$, where $i$ runs over all $1 \leq i \leq t$ such that $\left(R_{i}, \theta_{i}\right)$ is of type $\Gamma$. Then $R=\prod_{\Gamma} R(\Gamma)$, 
$\theta=\prod_{\Gamma} \theta(\Gamma), C=\prod_{\Gamma} C(\Gamma)$, and $\prod_{\Gamma} t(\Gamma)$ is a primary decomposition of $s$ in $G$. We may suppose $s(\Gamma)=t(\Gamma)$, so that $N(\theta)=\prod_{\Gamma} N(\theta(\Gamma))$, where $N(\theta(\Gamma))$ is the stabilizer of $\theta(\Gamma)$ in $N_{\mathrm{U}(V(\Gamma))}(R(\Gamma))$.

Each $\psi=\prod_{\Gamma} \psi(\Gamma)$, for $\psi \in \operatorname{Irr}^{0}(N(\theta), \theta)$ and $\psi(\Gamma) \in \operatorname{Irr}^{0}(N(\theta(\Gamma)), \theta(\Gamma))$. Let $b(\Gamma)$ be a block of $C(\Gamma)$ containing $\theta(\Gamma)$, and $B(\Gamma)=b(\Gamma)^{\mathrm{U}(V(\Gamma))}$. Then $B(\Gamma)$ is labeled by $(s(\Gamma),-)$ and $(R(\Gamma), I(\psi(\Gamma)))$ is a $B(\Gamma)$-weight. Conversely, if $B(\Gamma)$ is a block of $\mathrm{U}(V(\Gamma))$ with label $(s(\Gamma),-)$ and $(R(\Gamma), \varphi(\Gamma))$ is a $B(\Gamma)$-weight, then there exists a block $b(\Gamma)$ of $C(\Gamma) R(\Gamma)$ with defect group $R(\Gamma)$ and the canonical character $\theta(\Gamma)$ such that $b(\Gamma)^{\mathrm{U}(V(\Gamma))}=B(\Gamma)$ and $\varphi(\Gamma)=I(\psi(\Gamma))$ for some $\psi(\Gamma) \in \operatorname{Irr}^{0}(N(\theta(\Gamma)), \theta(\Gamma))$. Let $R=\prod_{\Gamma} R(\Gamma)$, $\theta=\prod_{\Gamma} \theta(\Gamma), b=\prod_{\Gamma} b(\Gamma)$, and $\psi=\prod_{\Gamma} \psi(\Gamma)$. Then $\psi \in \operatorname{Irr}^{0}(N(\theta), \theta)$, $b^{G}=B$, and $(R, I(\psi))$ is a $B$-weight. By $(4 \mathrm{~B})$ the number of $B(\Gamma)$-weights of $\mathrm{U}(V(\Gamma))$ is $f_{\Gamma}$ and so the number of $B$-weights of $G$ is $\Pi_{\Gamma} f_{\Gamma}$. By [3, (1A)] $f_{\Gamma}$ is also the number of $e_{\Gamma}$-tuples $\left(\kappa_{1}, \kappa_{2}, \ldots, \kappa_{e_{\Gamma}}\right)$ of partitions $\kappa_{i}$ such that $\sum_{i=1}^{e_{\Gamma}}\left|\kappa_{i}\right|=w_{\Gamma}$. This last number is also the number of partitions with $e_{\Gamma}$-core $\kappa_{\Gamma}$ and $e_{\Gamma}$-weight $w_{\Gamma}$. So $\prod_{\Gamma} f_{\Gamma}$ is the number $l(B)$ of irreducible modular characters in $B$ by $[11,(8 \mathrm{~A})]$. This completes the proof.

(4E). Let $q$ be a power of an odd prime, $V$ be a symplectic or even dimensional orthogonal space over $\mathbb{F}_{q}, G=I(V), G_{0}=I_{0}(V), B$ a block of $G$ with defect group $D$ and root block b such that $[V, D]=V$ and $\mathbf{b}^{G_{0}} \subseteq \mathscr{E}_{r}\left(G_{0},(s)\right)$ for some $s \in G_{0}^{*}$. Let $s^{*}$ be a dual of $s$ in $G_{0}$ and $m_{\Gamma}\left(s^{*}\right)=w_{\Gamma} \beta_{\Gamma} e_{\Gamma}$, where $w_{\Gamma}$ is an integer and $\beta_{\Gamma}=1$ or 2 according as $\Gamma \in \mathscr{F}_{1} \cup \mathscr{F}_{2}$ or $\Gamma \in \mathscr{F}_{0}$. Then the number of $B$-weights is $\Pi_{\Gamma} f_{\Gamma}$, where $f_{\Gamma}$ is given by (4B). In particular, the number $f_{\Gamma}$ is the numbler of $\beta_{\Gamma} e_{\Gamma}$-tuples $\left(\kappa_{1}, \kappa_{2}, \ldots, \kappa_{\beta_{\Gamma} e_{\Gamma}}\right)$ of partitions $\kappa_{i}$ such that $\sum_{i=1}^{\beta_{\Gamma} e_{\Gamma}}\left|\kappa_{i}\right|=w_{\Gamma}$.

Proof. Let $(R, \varphi)$ be a $B$-weight of $G, C=C_{G}(R)$, and $N=N_{G}(R)$. Then there is a block $b$ of $C R$ with defect group $R$ and the canonical character $\theta$ such that $b^{G}=B$ and $\varphi=I(\psi)$ for some $\psi \in \operatorname{Irr}^{0}(N(\theta), \theta)$. We may suppose $Z(D) \leq Z(R) \leq R \leq D$, so that $[V, R]=V$.

Let $R=\prod_{i}^{t} R_{i}$ and $V=\sum_{i=1}^{t} V_{i}$ be the decompositions of (2D), and let $C=\prod_{i=1}^{t} C_{i}$, and $\theta=\prod_{i=1}^{t} \theta_{i}$, where $C_{i}=C_{I\left(V_{i}\right)}\left(R_{i}\right)$ and $\theta_{i}$ is a character of $C_{i} R_{i}$ for all $i$. The block $b_{i}$ of $C_{i} R_{i}$ containing $\theta_{i}$ has defect group $R_{i}$. We claim that there is a weight $\left(R_{i}, \chi_{i}\right)$ of $I\left(V_{i}\right)$ such that $\chi_{i}$ covers $\theta_{i}$, namely there is an irreducible character $\chi_{i}$ of $N_{i} / R_{i}$ which covers $\theta_{i}$ and whose defect is 0 , where $N_{i}=N_{I\left(V_{i}\right)}\left(R_{i}\right)$. Thus by $(3 \mathrm{~J})(\mathrm{a})\left(R_{i}, \theta_{i}\right)$ has type $\Gamma$ for some $\Gamma \in \mathscr{F}^{\prime}$. To prove the claim we rewrite the decomposition of $R$ as $\prod_{j=1}^{u} R_{j}^{d_{j}}$, where $R_{j}$ 's are distinct basic subgroups and $R_{j}$ appears $d_{j}$-times as a component of $R$. Then

$$
N=\prod_{j=1}^{u} N_{j} \backslash \mathbf{S}\left(d_{j}\right)
$$

Thus $\varphi=\prod_{j=1}^{u} \varphi_{j}$ and $\left(R_{j}^{d_{j}}, \varphi_{j}\right)$ is a weight of $I\left(U_{j}\right)$, where $U_{j}$ is the underlying space of $R_{j}^{d_{j}}$. So we may suppose $u=1$ and $d=d_{1}$. Thus $R=R_{1}^{d}$, $N=N_{1} \backslash \mathbf{S}(d)$, and $\varphi$ is a character of defect 0 of $N / R \simeq\left(N_{1} / R_{1}\right) \backslash \mathbf{S}(d)$. As shown in the proof of $(4 \mathrm{C})$, the restriction of $\varphi$ to the base group $\left(N_{1} / R_{1}\right)^{d}$ of 
$N / R$ has a constituent $\left(\xi_{1}, \xi_{2}, \ldots, \xi_{d}\right)$ covering $\theta$ and each $\xi_{i}$ has defect 0 as character of $N_{1} / R_{1}$. Thus $\xi_{i}$ covers $\theta_{i}$ and the claim holds.

Let $\left(R_{i}, t_{i},-\right)$ be the label of Brauer pair $\left(R_{i}, b_{i}\right)$. As shown in the proof of (4B), $\left(R, \prod_{i=1}^{t} t_{i},-\right)$ is a label of $(R, b)$ and $b^{G_{0}} \subseteq \mathscr{E}_{r}\left(G_{0},\left(\prod_{i} t_{i}\right)\right)$. If $V^{*}$ is the underlying space of $G_{0}^{*}$, then $s$ and $\prod_{i=1}^{t} t_{i}$ are conjugate in $I\left(V^{*}\right)$ by (3K).

Let $R(\Gamma)=\prod_{i} R_{i}, V(\Gamma)=\sum_{i} V_{i}, C(\Gamma)=\prod_{i} C_{i}, \theta(\Gamma)=\prod_{i} \theta_{i}$, and $t(\Gamma)=\prod_{i} t_{i}$, where $i$ runs over $1 \leq i \leq t$ such that $\left(R_{i}, \theta_{i}\right)$ is of type $\Gamma$. Then $R=\prod_{\Gamma} R(\Gamma), V=\sum_{\Gamma} V(\Gamma), C=\prod_{\Gamma} C(\Gamma), \theta=\prod_{\Gamma} \theta(\Gamma)$, and $\prod_{\Gamma} t(\Gamma)$ is conjugate with $s$ in $I\left(V^{*}\right)$. It is clear that $N(\theta)=\prod_{\Gamma} N(\theta(\Gamma))$, where $N(\theta(\Gamma))$ is the stabilizer of $\theta(\Gamma)$ in $N_{I(V(\Gamma))}(R(\Gamma))$. A similar proof to the last paragraph of (4D) shows that the number of $B$-weights is $\prod_{\Gamma} f_{\Gamma}$ and by [3,(1A)] $f_{\Gamma}$ is the number of $\beta_{\Gamma} e_{\Gamma}$-tuples $\left(\kappa_{1}, \kappa_{2}, \ldots, \kappa_{\beta_{\Gamma} e_{\Gamma}}\right)$ of partitions $\kappa_{i}$ such that $\sum_{i}\left|\kappa_{i}\right|=w_{\Gamma}$. This completes the proof.

Remark. With the assumption of (4E), let $G=\mathrm{O}(V), G_{0}=\mathrm{SO}(V),(R, \varphi)$ a $B$-weight of $G$, and $\theta$ an irreducible character of $C=C_{G}(R)$ covered by $\varphi$. Then $\left|N(\theta): N_{0}(\theta)\right|=1$ of 2 according as $m_{X \pm 1}(s)=0$ or $m_{X \pm 1}(s) \neq 0$. Moreover, for each $\psi \in \operatorname{Irr}^{0}(N(\theta), \theta)$, the restriction $\left.\psi\right|_{N_{0}(\theta)}$ is irreducible, where $N_{0}(\theta)=N(\theta) \cap G_{0}$. Indeed in the notation above $R=\prod_{\Gamma} R(\Gamma)$, $V=\sum_{\Gamma} V(\Gamma), \theta=\prod_{\Gamma} \theta(\Gamma), N(\theta)=\prod_{\Gamma} N(\theta(\Gamma))$, and $s=\prod_{\Gamma} t(\Gamma)$. Thus $\psi=\prod_{\Gamma} \psi(\Gamma)$ for some $\psi(\Gamma) \in \operatorname{Irr}^{0}(N(\theta(\Gamma)), \theta(\Gamma))$. Since $[V, R]=V$, it follows that $[V(\Gamma), R(\Gamma)]=V(\Gamma)$. If $b(\Gamma)$ is the block of $C_{\mathrm{O}(V(\Gamma))}(R(\Gamma)) R(\Gamma)$ containing $\theta(\Gamma)$, then the Brauer pair $(R(\Gamma), \theta(\Gamma))$ has label $(R(\Gamma), t(\Gamma),-)$. By (4C) $\left|N(\theta(\Gamma)): N_{0}(\theta(\Gamma))\right|=\beta_{\Gamma}$ and $\left.\psi(\Gamma)\right|_{N_{0}(\theta(\Gamma))}$ is irreducible, where $N_{0}(\theta(\Gamma))=N(\theta(\Gamma)) \cap \operatorname{SO}(V(\Gamma))$. So $\left|N(\theta): N_{0}(\theta)\right|=1$ or 2 according as $m_{X \pm 1}(s)=0$ or $m_{X \pm 1}(s) \neq 0$, and $\left.\psi\right|_{N_{0}(\theta)}$ is irreducible.

(4F). Let $q$ be a power of an odd prime, $G=\mathrm{Sp}(2 n, q)=\mathrm{Sp}(V), B$ a block of $G$ contained in $\mathscr{E}_{r}(G,(s))$ for some semisimple $r^{\prime}$-element $s$ of $G^{*}=$ $\mathrm{SO}(2 n+1, q)$. Let $D$ be a defect group of $B, V_{0}=C_{V}(D), V_{+}=[V, D]$, so that $V=V_{0} \perp V_{+}$, and let $s=s_{0} \times s_{+}$be the corresponding decomposition in $G^{*}$. Then $m_{\Gamma}(s)-m_{\Gamma}\left(s_{0}\right)=w_{\Gamma} \beta_{\Gamma} e_{\Gamma}$ for some $w_{\Gamma} \geq 0$, where $\beta_{\Gamma}=1$ or 2 according as $\Gamma \in \mathscr{F}_{1} \cup \mathscr{F}_{2}$ or $\Gamma \in \mathscr{F}_{0}$. The number of B-weights is $\Pi_{\Gamma} f_{\Gamma}$, where $f_{\Gamma}$ is given by (4B). In particular, $f_{\Gamma}$ is the number of $\beta_{\Gamma} e_{\Gamma}$-tuples $\left(\kappa_{1}, \kappa_{2}, \ldots, \kappa_{\beta_{\Gamma} e_{\Gamma}}\right)$ of partitions $\kappa_{i}$ such that $\sum_{i=1}^{\beta_{\Gamma} e_{\Gamma}}\left|\kappa_{i}\right|=w_{\Gamma}$.

Proof. Let $(D, \mathbf{b})$ be a maximal Brauer pair of $G$ containing $(1, B)$, and $\vartheta$ be the canonical character of $\mathbf{b}$. Then $D=D_{0} \times D_{+}, \mathbf{b}=\mathbf{b}_{0} \times \mathbf{b}_{+}$, and $\vartheta=\vartheta_{0} \times \vartheta_{+}$, where $D_{0}=\left\langle 1_{V_{0}}\right\rangle \leq \operatorname{Sp}\left(V_{0}\right), D_{+} \leq \operatorname{Sp}\left(V_{+}\right), \mathbf{b}_{0}, \mathbf{b}_{+}$are blocks of $\operatorname{Sp}\left(V_{0}\right)$ and $C_{\mathrm{Sp}\left(V_{+}\right)}\left(D_{+}\right)$respectively, and $\vartheta_{0} \in \mathbf{b}_{0}, \vartheta_{+} \in \mathbf{b}_{+}$.

Let $(R, \varphi)$ be a $B$-weight of $G, C=C_{G}(R)$, and $N=N_{G}(R)$. Then there is a block $b$ of $C R$ with defect group $R$ and canonical character $\theta$ such that $b^{G}=B$ and $\varphi=I(\psi)$ for some $\psi \in \operatorname{Irr}^{0}(N(\theta), \theta)$. We may suppose $Z(D) \leq Z(R) \leq R \leq D$. Thus $C_{V}(R)=V_{0},[V, R]=V_{+}$, so that $R=R_{0} \times R_{+}, C=C_{0} \times C_{+}, N=N_{0} \times N_{+}$, where $R_{0}=D_{0}, R_{+} \leq \operatorname{Sp}\left(V_{+}\right)$, $C_{0}=N_{0}=\mathrm{Sp}\left(V_{0}\right), C_{+}=C_{\mathrm{Sp}\left(V_{+}\right)}\left(R_{+}\right)$, and $N_{+}=N_{\mathrm{Sp}\left(V_{+}\right)}\left(R_{+}\right)$. Let $b=b_{0} \times b_{+}$ and $\theta=\theta_{0} \times \theta_{+}$be the corresponding decompositions. Then $b_{0}$ is a block of $C_{0} R_{0}=\mathrm{Sp}\left(V_{0}\right)$ of defect $0, b_{+}$is a block of $C_{+} R_{+}$with defect group $R_{+}$, and $\theta_{0} \in b_{0}, \theta_{+} \in b_{+}$. We claim $\theta_{0}=\vartheta_{0}$. Indeed let $\left(D_{+}^{\prime}, \mathbf{b}_{+}^{\prime}\right)$ be a 
maximal Brauer pair of $\mathrm{Sp}\left(V_{+}\right)$containing $\left(R_{+}, b_{+}\right), \mathbf{b}^{\prime}=b_{0} \times \mathbf{b}_{+}^{\prime}$, and $D^{\prime}=$ $D_{0} \times D_{+}^{\prime}$. Then $\left(D^{\prime}, \mathbf{b}^{\prime}\right)$ is a maximal Brauer pair of $\operatorname{Sp}\left(V_{0}\right) \times \operatorname{Sp}\left(V_{+}\right)$containing $(R, b)$. If $N\left(D^{\prime}, \mathbf{b}^{\prime}\right)$ is the stabilizer of $\left(D^{\prime}, \mathbf{b}^{\prime}\right)$ in the normalizer $N_{G}\left(D^{\prime}\right)$ of $D^{\prime}$, then $\left(D^{\prime}, \mathbf{b}^{\prime}\right)$ is maximal in $G$ if and only if $\left(D^{\prime}, \mathbf{b}^{\prime}\right)$ is maximal in $N\left(D^{\prime}, \mathbf{b}^{\prime}\right)$. Since $N_{G}\left(D^{\prime}\right) \leq \operatorname{Sp}\left(V_{0}\right) \times \operatorname{Sp}\left(V_{+}\right),\left(D^{\prime}, \mathbf{b}^{\prime}\right)$ is maximal in $N\left(D^{\prime}, \mathbf{b}^{\prime}\right)$ and then maximal in $G$ containing $(1, B)$. By the Brauer First Main Theorem, $(D, \mathbf{b})^{g}=\left(D^{\prime}, \mathbf{b}^{\prime}\right)$ for some $g \in G$, so that $\left(\vartheta_{0} \times \vartheta_{+}\right)^{g}=\theta_{0} \times \vartheta_{+}^{\prime}$, where $\vartheta_{+}^{\prime}$ is the canonical charcter of $\mathbf{b}_{+}^{\prime}$. Since $D=D_{0} \times D_{+}$and $D^{\prime}=D_{0} \times D_{+}^{\prime}$, it follows $g \in \mathrm{Sp}\left(V_{0}\right) \times \mathrm{Sp}\left(V_{+}\right)$, and so $g=g_{0} \times g_{+}$, for $g_{0} \in \mathrm{Sp}\left(V_{0}\right)$ and $g_{+} \in \operatorname{Sp}\left(V_{+}\right)$. Thus $\theta_{0}=\vartheta_{0}$ and $\vartheta_{+}^{g_{+}}=\vartheta_{+}^{\prime}$. Moreover, $b_{+}^{\mathrm{Sp}\left(V_{+}\right)}=\mathbf{b}_{+}^{\mathrm{Sp}\left(V_{+}\right)}=\mathbf{b}_{+}^{\mathbf{S p}\left(V_{+}\right)}$.

It is clear that $N(\theta)=N_{0} \times N\left(\theta_{+}\right)$, where $N_{0}=\operatorname{Sp}\left(V_{0}\right)$ and $N\left(\theta_{+}\right)$is the stabilizer of $\theta_{+}$in $N_{+}$. If $\psi \in \operatorname{Irr}^{0}(N(\theta), \theta)$, then $\psi=\psi_{0} \times \psi_{+}$, where $\psi_{0}$ is an irreducible character of $N_{0}=C_{0}$ covering $\theta_{0}$, and $\psi_{+} \in \operatorname{Irr}^{0}\left(N\left(\theta_{+}\right), \theta_{+}\right)$, so that $\psi_{0}=\theta_{0}=\vartheta_{0}$. The correspondence $(R, I(\psi)) \mapsto\left(R_{+}, I_{+}\left(\psi_{+}\right)\right)$, where $\psi=\theta_{0} \times \psi_{+}$and $I_{+}\left(\psi_{+}\right)=\operatorname{Ind}_{N\left(\theta_{+}\right)}^{N_{+}}\left(\psi_{+}\right)$, is clearly a bijection form $\left\{(R, I(\psi)): \psi \in \operatorname{Irr}^{0}(N(\theta), \theta)\right\}$ to $\left\{\left(R_{+}, I_{+}\left(\psi_{+}\right)\right): \psi_{+} \in \operatorname{Irr}^{0}\left(N\left(\theta_{+}\right), \theta_{+}\right)\right\}$. Since $\left(R_{+}, I_{+}\left(\psi_{+}\right)\right)$is a $\mathbf{b}_{+}^{\mathrm{Sp}\left(V_{+}\right)}$-weight, the number of $B$-weights in $G$ is the number of $b_{+}^{\mathrm{Sp}\left(V_{+}\right)}$-weights in $\mathrm{Sp}\left(V_{+}\right)$. Thus $(4 \mathrm{E})$ implies $(4 \mathrm{~F})$.

In the following, we consider special orthogonal groups. If $G=$ $\mathrm{SO}(2 n+1, q)$, then by Fong and Srinivasan, [12, (10B)], a block $B$ of $G$ is labeled by a pair $(s, \kappa)$, where $s$ is a semisimple $r^{\prime}$-element in a dual group $G^{*}$ of $G, \kappa=\prod_{\Gamma} \kappa_{\Gamma}$ is a product of symbols or partitions $\kappa_{\Gamma}$ according as $\Gamma \in \mathscr{F}_{0}$ or $\Gamma \in \mathscr{F}_{1} \cup \mathscr{F}_{2}$ such that each $\kappa_{\Gamma}$ is the $e_{\Gamma}$-core of either a symbol with rank $\left[\frac{1}{2} m_{\Gamma}(s)\right]$ and odd defect, or a partition of $m_{\Gamma}(s)$ according as $\Gamma \in \mathscr{F}_{0}$ or $\Gamma \in \mathscr{F}_{1} \cup \mathscr{F}_{2}$. Moreover, by $[12,(12 \mathrm{~A})], B \subseteq \mathscr{E}_{r}(G,(s))$.

(4G). Let $q$ be a power of an odd prime, $G=\mathrm{SO}(V)=\operatorname{SO}(2 n+1, q), B$ a block of $G$ with label $(s, \kappa), \prod_{\Gamma} s(\Gamma)$ a primary decomposition of $s$ in $G^{*}=$ $\operatorname{Sp}(2 n, q)$, and let $w_{\Gamma}$ be an integer such that $m_{\Gamma}(s)=\left|\kappa_{\Gamma}\right|+e_{\Gamma} w_{\Gamma}$ if $\Gamma \in$ $\mathscr{F}_{1} \cup \mathscr{F}_{2}$, and $m_{\Gamma}(s)=2$ rank $\kappa_{\Gamma}+2 e_{\Gamma} w_{\Gamma}$ if $\Gamma \in \mathscr{F}_{0}$. Then the following hold:

(1) The number of $B$-weights of $G$ is $\Pi_{\Gamma} f_{\Gamma}$, where $f_{\Gamma}$ is the number of $\beta_{\Gamma} e_{\Gamma}$-tuples $\left(\kappa_{1}, \kappa_{2}, \ldots, \kappa_{\beta_{\Gamma} e_{\Gamma}}\right)$ of partitions $\kappa_{i}$ such that $\sum_{i=1}^{\beta_{\Gamma} e_{\Gamma}}\left|\kappa_{i}\right|=w_{\Gamma}$, and $\beta_{\Gamma}=1$ or 2 according as $\Gamma \in \mathscr{F}_{1} \cup \mathscr{F}_{2}$ or $\Gamma \in \mathscr{F}_{0}$.

(2) The number of $B$-weights of $G$ is $|B \cap \mathscr{E}(G,(s))|$.

Proof. Let $\widetilde{G}=\mathrm{O}(V)$, so that $\widetilde{G}=\left\langle-1_{V}\right\rangle \times G$, and let $\widetilde{B}=1 \times B$ be a block of $\widetilde{G}$, where 1 is the principal block of $\left\langle-1_{V}\right\rangle$. Let $(R, \varphi)$ be a $B$-weight of $G$, $N=N_{G}(R)$, and $\tilde{N}=N_{\widetilde{G}}(R)$, so that $\tilde{N}=\left\langle-1_{V}\right\rangle \times N$. There exists a block $b$ of $N$ such that $\varphi \in b$ and $b^{G}=B$. Let $\tilde{b}=1 \times b$ and $\tilde{\varphi}=1_{\left\langle-1_{V}\right\rangle} \times \varphi$, where $1_{\left\langle-1_{V}\right\rangle}$ is the principal character of $\left\langle-1_{V}\right\rangle$. Thus $\tilde{\varphi} \in \tilde{b}, \tilde{b} \widetilde{G}=\widetilde{B}$, and $(R, \tilde{\varphi})$ is a $\widetilde{B}$-weight of $\widetilde{G}$. The correspondence $(R, \varphi) \mapsto(R, \tilde{\varphi})$ is clearly a bijection from $B$-weights to $\widetilde{B}$-weights. Thus the number of $B$-weights in $G$ is the number of $\widetilde{B}$-weights in $\widetilde{G}$.

Let $(D, \tilde{\mathbf{b}})$ be a maximal Brauer pair of $\widetilde{G}$ containing $(1, \widetilde{B}), \tilde{\vartheta}$ the canonical character of $\tilde{\mathbf{b}}, V_{0}=C_{V}(D), V_{+}=[V, D]$. Then $V=V_{0} \perp V_{+}$and $V_{+}$is an even dimensional orthogonal space since $D$ is radical. In addition, let $\widetilde{G}_{0}=\mathrm{O}\left(V_{0}\right), G_{0}=\mathrm{SO}\left(V_{0}\right), \widetilde{G}_{+}=\mathrm{O}\left(V_{+}\right)$, and $G_{+}=\mathrm{SO}\left(V_{+}\right)$. Then 
$D=D_{0} \times D_{+}, \tilde{\mathbf{b}}=\mathbf{b}_{0} \times \tilde{\mathbf{b}}_{+}, \tilde{\vartheta}=\tilde{\vartheta}_{0} \times \tilde{\vartheta}_{+}$, where $D_{0}=\left\langle 1_{V_{0}}\right\rangle \leq \widetilde{G}_{0}, D_{+} \leq \tilde{G}_{+}$, $\tilde{\mathbf{b}}_{0}, \tilde{\mathbf{b}}_{+}$are blocks of $\widetilde{G}_{0}, C_{\widetilde{G}_{+}}\left(D_{+}\right)$respectively, and $\tilde{\vartheta}_{0} \in \tilde{\mathbf{b}}_{0}, \tilde{\vartheta}_{+} \in \tilde{\mathbf{b}}_{+}$.

Now the proof of $(4 \mathrm{~F})$ can be applied here with $G$ replaced by $\widetilde{G}, B$ by $\widetilde{B}, \vartheta$ by $\tilde{\vartheta}, \mathbf{b}$ by $\tilde{\mathbf{b}}$, and some obvious modifications. Thus the number of $\widetilde{B}$-weights in $\widetilde{G}$ is the number of $\tilde{\mathbf{b}}_{+} \widetilde{G}_{+}$-weights in $\widetilde{G}_{+}$. Moreover, $\tilde{\mathbf{b}}_{+}$is a root block of $\tilde{\mathbf{b}}_{+}^{\widetilde{G}_{+}}$and $\tilde{\mathbf{b}}^{G_{+}} \subseteq \mathscr{E}_{r}\left(G_{+},\left(s_{+}\right)\right)$. Since $C_{\widetilde{G}}(D)=\left\langle-1_{V_{0}}\right\rangle \times C_{G}(D)$ and $\widetilde{B}=1 \times B$, it follows that $\tilde{\mathbf{b}}=1 \times \mathbf{b}$ for some block $\mathbf{b}$ of $C_{G}(D)$ and $\tilde{\mathbf{b}}_{0}=1 \times \mathbf{b}_{0}$, where 1 is the principal block of $\left\langle-1_{V_{0}}\right\rangle$ and $\mathbf{b}_{0}$ is a block of $G_{0}$. Thus $\tilde{\vartheta}_{0}=1_{\left\langle-1_{v_{0}}\right\rangle} \times \vartheta_{0}$ for $\vartheta_{0} \in \mathbf{b}_{0}$. Since $C_{\widetilde{G}_{+}}\left(D_{+}\right)=C_{G_{+}}\left(D_{+}\right), \tilde{\mathbf{b}}_{+}$is a block of $C_{G_{+}}\left(D_{+}\right)$and then $\mathbf{b}_{0} \times \tilde{\mathbf{b}}_{+}$is a root block of $B$. Here $\mathbf{b}_{0} \times \tilde{\mathbf{b}}_{+}$is regarded as a block of $C_{G}(D) D$. As shown in the proof of $[12,(12 \mathrm{~A})],\left(s_{0}, \kappa\right)$ is the label of $\vartheta_{0}$, so that $m_{\Gamma}(s)=\left|\kappa_{\Gamma}\right|+m_{\Gamma}\left(s_{+}\right)$if $\Gamma \in \mathscr{F}_{1} \cup \mathscr{F}_{2}$, and $m_{\Gamma}(s)=2$ rank $\kappa+m_{\Gamma}\left(s_{+}\right)$if $\Gamma \in \mathscr{F}_{0}$. Thus $m_{\Gamma}\left(s_{+}\right)=m_{\Gamma}\left(s_{+}^{*}\right)=w_{\Gamma} \beta_{\Gamma} e_{\Gamma}$, where $s_{+}^{*}$ is a dual of $s_{+}$in $G_{+}$. So $(4 \mathrm{G})(1)$ follows from (4E).

Finally, there exists a bijection between $\mathscr{E}(G,(s))$ and $\mathscr{E}\left(C_{G^{*}}(s)^{*},(1)\right)$. By $[12,(12 \mathrm{~A})]$ and [19, Proposition 14] the number given by (1) is the number of the characters of $\mathscr{E}(G,(s)) \cap B$.

Remark. (1) Suppose $G=\mathrm{SO}(2 n+1, q)$ and $r$ is a good prime. Then by [13, 5.1] $l(B)=|B \cap \mathscr{E}(G,(s))|$, so that $l(B)$ is the number of $B$-weights.

(2) By a result of Fong and Olsson (unpublished), if $G=\mathrm{SO}(2 n+1, q)$ and $r$ is odd, then $l(B)=|B \cap \mathscr{E}(G,(s))|$ and this is the number of $B$-weights.

$(4 \mathrm{H})$. Let $q$ be a power of an odd prime, $G=\mathrm{SO}^{ \pm}(2 n, q)=\mathrm{SO}(V), B$ is a block of $G$ with defect group $D$ and root block $\mathbf{b}$ such that $B \subseteq \mathscr{E}_{r}(G,(s))$ for some semisimple $r^{\prime}$-element $s$ of $G^{*}=\operatorname{SO}^{ \pm}(2 n, q)$, and let $V_{0}=C_{V}(D)$, $V_{+}=[V, D]$, so that $V=V_{0} \perp V_{+}$. Let $s=s_{0} \times s_{+}, \vartheta=\vartheta_{0} \times \vartheta_{+}$be the corresponding decompositions, where $\vartheta$ is the canonical character of $\mathbf{b}$. If $m_{\Gamma}\left(s_{+}\right)=w_{\Gamma} \beta_{\Gamma} e_{\Gamma}$ for some $w_{\Gamma} \geq 0$, then denote $f_{\Gamma}$ the number of $\beta_{\Gamma} e_{\Gamma}$-tuples $\left(\kappa_{1}, \kappa_{2}, \ldots, \kappa_{\beta_{\Gamma} e_{\Gamma}}\right)$ of partitions $\kappa_{i}$ such that $\sum_{i=1}^{\beta_{\Gamma} e_{\Gamma}}\left|\kappa_{i}\right|=w_{\Gamma}$, where $\beta_{\Gamma}=1$ or 2 according as $\Gamma \in \mathscr{F}_{1} \cup \mathscr{F}_{2}$ or $\Gamma \in \mathscr{F}_{0}$. Then the following hold:

(1) If either $m_{X \pm 1}\left(s_{+}\right)=0$ or $\vartheta_{0}^{\sigma_{0}}=\vartheta_{0}$ for some $\sigma_{0} \in \mathrm{O}\left(V_{0}\right)$ of determinant -1 , then the number of $B$-weights is $\prod_{\Gamma} f_{\Gamma}$.

(2) Suppose $m_{X \pm 1}\left(s_{+}\right) \neq 0$. If either $V_{0}=0$ or $\vartheta_{0}^{\sigma_{0}} \neq \vartheta_{0}$ for any $\sigma_{0} \in \mathrm{O}\left(V_{0}\right)$ of determinant -1 , then the number of $B$-weights is $\frac{1}{2} \prod_{\Gamma} f_{\Gamma}$.

Proof. Let $\widetilde{G}=\mathrm{O}(V), \widetilde{G}_{0}=\mathrm{O}\left(V_{0}\right), G_{0}=\mathrm{SO}\left(V_{0}\right), \widetilde{G}_{+}=\mathrm{O}\left(V_{+}\right), G_{+}=$ $\mathrm{SO}\left(V_{+}\right)$, and $D=D_{0} \times D_{+}$, where $D_{0}=\left\langle 1_{V_{0}}\right\rangle$ and $D_{+} \leq G_{+}$. In addition, let $\mathbf{b}_{+}$be a block of $C_{G_{+}}\left(D_{+}\right) D_{+}$containing $\vartheta_{+}$, and $\mathbf{b}_{+}^{G_{+}} \subseteq \mathscr{E}_{r}\left(G_{+},\left(s_{+}^{\prime}\right)\right)$ for some semisimple $r^{\prime}$-element $s_{+}^{\prime}$ of $G_{+}^{*}$. Then $\left(D_{+}, s_{+}^{\prime},-\right)$ is a label of Brauer pair $\left(D_{+}, \mathbf{b}_{+}\right)$. But $\left(D_{+}, s_{+},-\right)$is also a label of $\left(D_{+}, \mathbf{b}_{+}\right)$, and so $s_{+}, s_{+}^{\prime}$ are conjugate in $G_{+}^{*}$.

Let $(R, \varphi)$ be a $B$-weight, $C=C_{G}(R), \widetilde{C}=C_{\widetilde{G}}(R), N=N_{G}(R)$, and $\tilde{N}=$ $N_{\widetilde{G}}(R)$. Then there exists a block $b$ of $C R$ with defect group $R$ and canonical character $\theta$ such that $b^{G}=B$ and $\varphi=I(\psi)$ for some $\psi \in \operatorname{Irr}^{0}(N(\theta), \theta)$. We may suppose $Z(D) \leq Z(R) \leq R \leq D$, so that $R=R_{0} \times R_{+}, C=G_{0} \times C_{+}$, $\widetilde{C}=\widetilde{G}_{0} \times C_{+}, N=\left\langle\tau, G_{0} \times N_{+}\right\rangle$, and $\widetilde{N}=\widetilde{G}_{0} \times \widetilde{N}_{+}$, where $R_{0}=D_{0}$, 
$R_{+} \leq G_{+}, C_{+}=C_{G_{+}}\left(R_{+}\right), N_{+}=N_{G_{+}}\left(R_{+}\right), \tilde{N}_{+}=N_{\widetilde{G}_{+}}\left(R_{+}\right)$, and $\tau=\tau_{0} \times \tau_{+}$ with $\tau_{0} \in \widetilde{G}_{0}, \tau_{+} \in \widetilde{G}_{+}$of determinants -1 . Thus $\tilde{N}=\left\langle\tau_{0}, N\right\rangle, \theta=\theta_{0} \times \theta_{+}$, and $b=b_{0} \times b_{+}$, where $b_{0}$ is a block of $G_{0}$ of defect $0, b_{+}$is a block of $C_{+} R_{+}$ with defect group $R_{+}, \theta_{0} \in b_{0}$, and $\theta_{+} \in b_{+}$.

Let $\left(D_{+}^{\prime}, \mathbf{b}_{+}^{\prime}\right)$ be a maximal Brauer pair of $\widetilde{G}_{+}$containing $\left(R_{+}, b_{+}\right)$, where $b_{+}$is regarded as a block of $C_{+}$. Let $D^{\prime}=D_{0} \times D_{+}^{\prime}, \mathbf{b}^{\prime}=b_{0} \times \mathbf{b}_{+}^{\prime}$. A similar proof to that of $(4 \mathrm{~F})$ shows that $\left(D^{\prime}, \mathbf{b}^{\prime}\right)$ is a maximal Brauer pair of $G$ containing $(R, b)$, where $b$ is regarded as a block of $C$. So $(D, \mathbf{b})^{g}=\left(D^{\prime}, \mathbf{b}^{\prime}\right)$ for some $g \in G$ by the Brauer First Main Theorem. Thus $g=g_{0} \times g_{+}$for $g_{0} \in \widetilde{G}_{0}$ and $g_{+} \in \widetilde{G}_{+}$. If $\operatorname{det} g_{0}=-1$, then we replace $b$ by $b^{\tau}$ and $\theta_{0}$ by $\theta_{0}^{\tau_{0}}$. We may suppose $g_{0} \in G_{0}$ and $g_{+} \in G_{+}$. Since $\left(\vartheta_{0} \times \vartheta_{+}\right)^{g}=\theta_{0} \times \vartheta_{+}^{\prime}$, it follows that $\theta_{0}=\vartheta_{0}$ and $\vartheta_{+}^{g_{+}}=\vartheta_{+}^{\prime}$, where $\vartheta_{+}^{\prime}$ is the canonical character of $\mathbf{b}_{+}^{\prime}$. It follows that $\mathbf{b}_{+}^{\prime G_{+}}=\mathbf{b}_{+}^{G_{+}}$, so that $\mathbf{b}_{+}^{\prime \widetilde{G}_{+}}=b_{+}^{\widetilde{G}_{+}}$and we may suppose $\left(R_{+}, s_{+},-\right)$is a label of $\left(R_{+}, b_{+}\right)$. Replacing $R$ by $R_{0} \times R_{+}^{g_{+}^{-1}}$ and $b$ by $b_{0} \times b_{+}^{g_{+}^{-1}}$, we may suppose $(R, b) \leq(D, \mathbf{b})$.

(1) Suppose $m_{X \pm 1}\left(s_{+}\right)=0$. Set $\widetilde{B}_{+}=\mathbf{b}_{+}^{\widetilde{G}_{+}}$, so that $\mathbf{b}_{+}$is a root block of $\widetilde{B}_{+}$ and $D_{+}$is a defect group of $\widetilde{B}_{+}$. We shall show that the number of $B$-weights in $G$ is the number of $\widetilde{B}_{+}$-weights in $\widetilde{G}_{+}$.

Let $N\left(\theta_{+}\right)$and $\tilde{N}\left(\theta_{+}\right)$be the stabilizers of $\theta_{+}$in $N_{+}$and $\tilde{N}_{+}$respectively. By the remark of $(4 \mathrm{E}) N\left(\theta_{+}\right)=\tilde{N}\left(\theta_{+}\right)$. Since $N(\theta)=G_{0} \times N\left(\theta_{+}\right)$, it follows that $\psi=\theta_{0} \times \psi_{+}$for some $\psi_{+} \in \operatorname{Irr}^{0}\left(N\left(\theta_{+}\right), \theta_{+}\right)$. Then $\left(R_{+}, I_{+}\left(\psi_{+}\right)\right)$ is a $\widetilde{B}_{+}$-weight of $\widetilde{G}_{+}$, where $I_{+}\left(\psi_{+}\right)=\operatorname{Ind}{\widetilde{N}\left(\theta_{+}\right)}_{\tilde{N}_{+}}\left(\psi_{+}\right)$. Conversely, suppose $\left(R_{+}, \varphi_{+}\right)$is a $\widetilde{B}_{+}$-weight, where $R_{+}$is a radical subgroup of $\widetilde{G}_{+}$. Then $\left[V_{+}, R_{+}\right]=V_{+}$and there exists a block of $C_{+} R_{+}$with defect group $R_{+}$and canonical character $\theta_{+}$such that $\varphi_{+}=I_{+}\left(\psi_{+}\right)$for some $\psi_{+} \in \operatorname{Irr}^{0}\left(\tilde{N}\left(\theta_{+}\right), \theta_{+}\right)$ and $b_{+}^{\widetilde{G}_{+}}=\widetilde{B}_{+}$, where $C_{+}, \widetilde{N}_{+}$are given before, $\tilde{N}\left(\theta_{+}\right)$is the stabilizer of $\theta_{+}$ in $\tilde{N}_{+}$, and $I_{+}$is defined as before. By the remark of $(4 \mathrm{E}) \tilde{N}\left(\theta_{+}\right) \leq G_{+}$. Let $\theta=\vartheta_{+} \times \theta_{+}, R=D_{0} \times R_{+}, \psi=\vartheta_{0} \times \psi_{+}, b$ a block of $C_{G}(R)$ containing $\theta$, and $N(\theta)$ the stabilizer of $\theta$ in $N=N_{G}(R)$. Then $N(\theta)=G_{0} \times \tilde{N}\left(\theta_{+}\right)$and $\psi \in \operatorname{Irr}^{0}(N(\theta), \theta)$. We may suppose $\left(R_{+}, b_{+}\right) \leq\left(D_{+}, \mathbf{b}_{+}\right)$, so that $(R, b) \leq$ $(D, \mathbf{b})$. Thus $b^{G}=B$ and $(R, I(\psi))$ is a $B$-weight. The correspondence $(R, I(\psi)) \mapsto\left(R_{+}, I_{+}\left(\psi_{+}\right)\right)$, where $R=D_{0} \times R_{+}$and $\psi=\vartheta_{0} \times \psi_{+}$is clearly a bijection from $\left\{(R, I(\psi)): \psi \in \operatorname{Irr}^{0}(N(\theta), \theta)\right\}$ to $\left\{\left(R_{+}, I_{+}\left(\psi_{+}\right)\right): \psi_{+} \in\right.$ $\left.\operatorname{Irr}^{0}\left(\tilde{N}\left(\theta_{+}\right), \theta_{+}\right)\right\}$. So the number of $B$-weights is the number of $\widetilde{B}_{+}$-weights, and it is $\prod_{\Gamma} f_{\Gamma}$ by $(4 \mathrm{E})$.

Suppose $\vartheta_{0}^{\sigma_{0}}=\vartheta_{0}$ for some $\sigma_{0} \in \widetilde{G}_{0}$ of determinant -1 . Then there are two irreducible characters $\vartheta_{0}^{\prime}$ and $\vartheta_{0}^{\prime \prime}$ of $\widetilde{G}_{0}$ covering $\vartheta_{0}$. Let $\vartheta^{\prime}=\vartheta_{0}^{\prime} \times \vartheta_{+}, \vartheta^{\prime \prime}=$ $\vartheta_{0}^{\prime \prime} \times \vartheta_{+}$, and $\mathbf{b}^{\prime}, \mathbf{b}^{\prime \prime}$ be the blocks of $C_{\widetilde{G}}(D)$ containing $\vartheta^{\prime}, \vartheta^{\prime \prime}$ respectively. Then $\vartheta^{\prime}, \vartheta^{\prime \prime}$ are not conjugate in $N_{\widetilde{G}}(D)=\widetilde{G}_{0} \times N_{\widetilde{G}_{+}}\left(D_{+}\right)$, so $\mathbf{b}^{\prime \widetilde{G}}$ and $\mathbf{b}^{\prime \prime} \widetilde{G}$ are two blocks of $\widetilde{G}$. We shall show that the number of $\mathbf{b}^{\prime \widetilde{G}_{\text {-weights }}}$ is the number of $B$-weights.

Suppose $(R, \varphi)$ is a $B$-weight. In the notation above, $N=\left\langle\tau, G_{0} \times N_{+}\right\rangle$ 
and $\widetilde{N}=\left\langle\tau_{0}, G_{0} \times \widetilde{N}_{+}\right\rangle$, where $\tau=\tau_{0} \times \tau_{+}$with $\tau_{0} \in \widetilde{G}_{0}, \tau_{+} \in \widetilde{G}_{+}$of determinants -1 . Moreover, we may suppose $(R, b) \leq(D, \mathbf{b})$ and $\theta_{0}=\vartheta_{0}$. Let $\tilde{\theta}=\vartheta_{0}^{\prime} \times \theta_{+}$and $\tilde{b}$ the block of $\widetilde{C}$ containing $\tilde{\theta}$. Then $(R, \tilde{b}) \leq\left(D, \mathbf{b}^{\prime}\right)$ and $\tilde{b} \widetilde{G}=\mathbf{b}^{\prime} \widetilde{G}$. Conversely, if $(R, \tilde{\varphi})$ is a weight of $\mathbf{b}^{\prime} \widetilde{G}$, then there exists a block $\tilde{b}$ of $\tilde{C} R$ with defect group $R$ and canonical character $\tilde{\theta}$ such that $\tilde{b} \widetilde{G}=\mathbf{b}^{\widetilde{G}}$ and $\tilde{\varphi} \in \operatorname{Irr}(\tilde{N}, \tilde{\theta})$, where $\tilde{C}$ is defined before. Then $\tilde{b}=\tilde{b}_{0} \times b_{+}$ and $\tilde{\theta}=\tilde{\theta}_{0} \times \theta_{+}$, where $\tilde{b}_{0}$ and $b_{+}$are blocks of $\widetilde{G}_{0}$ and $C_{+}$respectively and $\tilde{\theta}_{0} \in \tilde{b}_{0}$ and $\theta_{+} \in b_{+}$. As shown in the proof of $(4 \mathrm{~F})$, we may suppose $\tilde{\theta}_{0}=\vartheta_{0}^{\prime}$ and $(R, \tilde{b}) \leq\left(D, \mathbf{b}^{\prime}\right)$. Let $\theta=\vartheta_{0} \times \theta_{+}$and $b$ the block of $C$ containing $\theta$. Then $(R, b) \leq(D, \mathbf{b})$. In addition, each character $\varphi \in \operatorname{Irr}^{0}(N, \theta)$ or $\tilde{\varphi} \in \operatorname{Irr}^{0}(\tilde{N}, \tilde{\theta})$ covers a character $\operatorname{of} \operatorname{Irr}^{0}\left(G_{0} \times N_{+}, \theta\right)$ and each character of $\operatorname{Irr}^{0}\left(G_{0} \times N_{+}, \theta\right)$ decomposes as $\vartheta_{0} \times \varphi_{+}$for some $\varphi_{+} \in \operatorname{Irr}^{0}\left(N_{+}, \theta_{+}\right)$. So it suffices to show that the number of $\mathbf{b}^{\prime \sigma^{G}}$-weights of the form $(R, \tilde{\varphi})$ with $\tilde{\varphi}$ covering $\vartheta_{0} \times \varphi_{+}$is the number of $B$-weights of the form $(R, \varphi)$ with $\varphi$ covering $\vartheta_{0} \times \varphi_{+}$. It is equivalent to show that the number of irreducible characters in $\tilde{b}^{\widetilde{N}}$ covering $\vartheta_{0} \times \varphi_{+}$is the number of irreducible characters in $h^{N}$ covering $\vartheta_{0} \times \varphi_{+}$since $(\tilde{N}: N)=\left(N: G_{0} \times N_{+}\right)=2$.

If $\tau_{+}$stabilizes $\varphi_{+}$, then there are two irreducible characters $\varphi_{+}^{\prime}$ and $\varphi_{+}^{\prime \prime}$ of $\tilde{N}_{+}$covering $\varphi_{+}$, so that there are four irreducible characters $\vartheta_{0}^{\prime} \times \varphi_{+}^{\prime}, \vartheta_{0}^{\prime} \times \varphi_{+}^{\prime \prime}$, $\vartheta_{0}^{\prime \prime} \times \varphi_{+}^{\prime}$, and $\vartheta_{0}^{\prime \prime} \times \varphi_{+}^{\prime \prime}$ of $\tilde{N}=\widetilde{G}_{0} \times \widetilde{N}_{+}$covering $\vartheta_{0} \times \varphi_{+}$. Moreover, exactly two of them $\vartheta_{0}^{\prime} \times \varphi_{+}^{\prime}$ and $\vartheta_{0}^{\prime} \times \varphi_{+}^{\prime \prime} \operatorname{cover} \vartheta_{0}^{\prime} \times \varphi_{+}$and both lie in $\tilde{b}^{\tilde{N}}$ by [10, V 3.10 and 3.7]. Since $\tau=\tau_{0} \times \tau_{+}$stabilizes $\vartheta_{0} \times \varphi_{+}$, there are two irreducible characters of $N$ covering $\vartheta_{0} \times \varphi_{+}$and lying in $b^{N}$. It follows that both $\tilde{b}^{\tilde{N}}$ and $b^{N}$ have two irreducible characters covering $\vartheta_{0} \times \varphi_{+}$, so that the number of $\mathbf{b}^{\widetilde{G}}$-weights is the number of $B$-weights.

If $\tau_{+}$does not stabilize $\varphi_{+}$, then there are two irreducible characters $\vartheta_{0}^{\prime} \times$ $\left(\varphi_{+}+\varphi_{+}^{\tau_{+}}\right)$and $\vartheta_{0}^{\prime \prime} \times\left(\varphi_{+}+\varphi_{+}^{\tau_{+}}\right)$of $\tilde{N}$ covering $\vartheta_{0} \times \varphi_{+}$and only the first lies in $\tilde{b}^{\widetilde{N}}$. Since $\left(\vartheta_{0} \times \varphi_{+}\right)^{\tau} \neq \vartheta_{0} \times \varphi_{+}, N$ has only one irreducible character covering $\vartheta_{0} \times \varphi_{+}$and lying in $b^{N}$. So both $\tilde{b}^{\widetilde{N}}$ and $b^{N}$ has one irreducible character covering $\vartheta_{0} \times \varphi_{+}$. Thus the number of $\mathbf{b}^{\prime{ }^{\prime}}$-weights is the number of $B$-weights.

A similar proof to that of $(4 \mathrm{~F})$ can be applied here with $G$ replaced by $\widetilde{G}$, $B$ by $\mathbf{b}^{\prime} \widetilde{G}, \mathbf{b}$ by $\mathbf{b}^{\prime}, \vartheta$ by $\vartheta^{\prime}$, and some obvious modifications, so that the number of $\mathbf{b}^{\prime \widetilde{G}}$-weights is the number of $\mathbf{b}_{+}^{\widetilde{G}_{+}}$-weights. By (4E) the number of $\widetilde{b}_{+} \widetilde{G}_{+}$-weights is $\prod_{\Gamma} f_{\Gamma}$ and this is the number of $B$-weights. This completes the proof of (1).

(2) Suppose $m_{X \pm 1}\left(s_{+}\right) \neq 0$ and $(R, \varphi)$ is a $B$-weight. In the notation above, suppose $\widetilde{N}(\theta)$ and $N(\theta)$ are the stabilizers of $\theta$ in $\widetilde{N}$ and $N$ respectively.

If $V_{0}=0$, then $(\tilde{N}(\theta): N(\theta))=2$ and $\left|\operatorname{Irr}^{0}(\tilde{N}(\theta), \theta)\right|=2\left|\operatorname{Irr}^{0}(N(\theta), \theta)\right|$ by the remark of (4E). So the number of $B$-weights is $\frac{1}{2} \prod_{\Gamma} f_{\Gamma}$ by (4E).

Suppose $V_{0} \neq 0$ and $\vartheta_{0}^{\tau_{0}} \neq \vartheta_{0}$ for some $\tau_{0} \in \widetilde{G}_{0}$ of determinant -1 . By the proof above, we may suppose $\theta=\vartheta_{0} \times \theta_{+}$for some character $\theta_{+}$of $C_{+}$ and $(R, b) \leq(D, \mathbf{b})$. Let $\widetilde{N}\left(\theta_{+}\right)$and $N\left(\theta_{+}\right)$be the stabilizers of $\theta_{+}$in $\tilde{N}_{+}$ and $N_{+}$respectively. Then $\tilde{N}(\theta)=G_{0} \times \tilde{N}\left(\theta_{+}\right)$and $N(\theta)=G_{0} \times N\left(\theta_{+}\right)$, 
so that by the remark of (4E), $\left|\operatorname{Irr}^{0}\left(\tilde{N}\left(\theta_{+}\right), \theta_{+}\right)\right|=2\left|\operatorname{Irr}^{0}\left(N\left(\theta_{+}\right), \theta_{+}\right)\right|$. Thus $\left|\operatorname{Irr}^{0}(\tilde{N}(\theta), \theta)\right|=2\left|\operatorname{Irr}^{0}(N(\theta), \theta)\right|$ since each character $\tilde{\psi}$ of $\operatorname{Irr}^{0}(\tilde{N}(\theta), \theta)$ and each $\psi$ of $\operatorname{Irr}^{0}(N(\theta), \theta)$ decomposes as $\tilde{\psi}=\vartheta_{0} \times \tilde{\psi}_{+}$and $\psi=\vartheta_{0} \times \psi_{+}$ for some $\tilde{\psi}_{+} \in \operatorname{Irr}^{0}\left(\tilde{N}\left(\theta_{+}\right), \theta_{+}\right)$and $\psi_{+} \in \operatorname{Irr}^{0}\left(N\left(\theta_{+}\right), \theta_{+}\right)$. Let $\mathbf{b}^{\prime}$ be the block of $C_{\widetilde{G}}(D) D$ containing $\vartheta^{\prime}=\left(\vartheta_{0}+\vartheta_{0}^{\tau_{0}}\right) \times \vartheta_{+}$and $\tilde{b}$ the block of $\widetilde{C}$ containing $\tilde{\theta}=\left(\vartheta_{0}+\vartheta_{0}^{\tau_{0}}\right) \times \theta_{+}$. Since $(R, b) \leq(D, \mathbf{b})$ in $G$, it follows that $(R, \tilde{b}) \leq\left(D, \mathbf{b}^{\prime}\right)$ in $\widetilde{G}$, so that $\tilde{b}^{\widetilde{G}}=\mathbf{b}^{\mathbf{\prime}} \widetilde{G}$. Thus the number of $B$-weights is half of the number of $\mathbf{b}^{{ }^{\prime}}$-weights. A similar proof to that of $(4 \mathrm{~F})$ can be applied here with $G$ replaced by $\widetilde{G}, B$ by $\mathbf{b}^{\widetilde{G}}, \mathbf{b}$ by $\mathbf{b}^{\prime}, \vartheta$ by $\vartheta^{\prime}$, and some obvious modifications, so that the number of $\mathbf{b}^{\prime \widetilde{G}_{+}}$-weights is the number of $\mathbf{b}_{+}^{\widetilde{G}}$-weights. By (4E) the number of $\mathbf{b}_{+}^{\widetilde{G}_{+}}$-weights is $\Pi_{\Gamma} f_{\Gamma}$ and so the number of $B$-weights is $\frac{1}{2} \prod_{\Gamma} f_{\Gamma}$. This completes the proof.

\section{REFERENCES}

1. J. L. Alperin, Large abelian subgroups of p-groups, Trans. Amer. Math. Soc. 117 (1965), 10-20.

2. Weights for finite groups, Arcata Conf. on Representations of Finite Groups, Proc. Sympos. Pure Math., vol. 47, Amer. Math. Soc., Providence, RI, 1987, pp. 369-379.

3. J. L. Alperin and P. Fong, Weights for symmetric and general linear groups, J. Algebra 131 (1990), 2-22.

4. Jianbei An, 2-weights for general linear groups, J. Algebra 149 (1992), 500-527.

5. _ 2-weights for unitary groups, Trans. Amer. Math. Soc. 339 (1993), 251-278.

6. R. Brauer, On blocks and sections. I, II, Amer. J. Math. 89 (1967), 1115-1136; 90 (1968), 895-925.

7. M. Broué, Les l-blocs des groupes $\mathrm{GL}(n, q)$ et $\mathrm{U}\left(n, q^{2}\right)$ et leurs structures locales, Sém. Bourbaki Astérisque 640 (1986), 159-188.

8. M. Broué and J. Michel, Blocs et séries de Lusztig dans un groupe réductif fini, J. Reine Angew. Math. 395 (1989), 56-67.

9. F. Digne and J. Michel, Foncteurs de Lusztig et charactéres des groups linéaires et unitaires sur corps fini, J. Algebra 107 (1987), 217-255.

10. W. Feit, The representation theory of finite groups, North-Holland, Amsterdam, 1982.

11. P. Fong and B. Srinivasan, The blocks of finite general linear and unitary groups, Invent. Math. 69 (1982), 109-153.

12. __ The blocks of finite classical groups, J. Reine Angew. Math. 396 (1989), 122-191.

13. M. Geck and G. Hiss, Basic sets of Brauer characters of finite groups of Lie type, J. Reine Angew. Math. 418 (1991), 173-188.

14. D. Gorenstein, Finite groups, Harper and Row, New York, 1968.

15. R. L. Griess, Automorphisms of extra special groups and nonvanishing degree 2 cohomology, Pacific J. Math. 48 (1973), 402-422.

16. I. M. Isaacs, Characters of solvable and symplectic groups, Amer. J. Math. 95 (1973), 594-635.

17. A. Kerber, Permutations of permutation groups. I, Lecture Notes in Math., vol. 240, SpringerVerlag, Berlin, Heidelberg, and New York, 1971.

18. G. Lusztig, Irreducible representations of finitc classical groups, Invent. Math. 43 (1977), 125-175. 
19. J. Olsson, Remarks on symbols, hooks, and degrees of unipotent characters, J. Combin. Theory Ser. A 42 (1986), 223-238.

20. D. L. Winter, The automorphism group of an extraspecial p-group, Rocky Mountain J. Math. 2 (1972), 159-168.

Department of Mathematics, University of Illinois at Chicago, Chicago, Illinois 60680

Current address: Department of Mathematics, University of Auckland, Auckland, New Zealand E-mail address: an@mat . aukuni .ac.nz 